\title{
On the generalized fractional snap boundary problems via $G$-Caputo operators: existence and stability analysis
}

\author{
Mohammad Esmael Samei ${ }^{1}$, Mohammed M. Matar ${ }^{2 *}$, Sina Etemad ${ }^{3}$ and Shahram Rezapour ${ }^{3,4^{*}}$ (ID
}

\author{
*Correspondence: \\ mohammed_mattar@hotmail.com; \\ sh.rezapour@azaruniv.ac.ir; \\ sh.rezapour@mail.cmuh.org.tw \\ rezapourshahram@yahoo.ca \\ 2Department of Mathematics, \\ Al-Azhar University-Gaza, Gaza Strip, \\ State of Palestine \\ ${ }^{3}$ Department of Mathematics, \\ Azarbaijan Shahid Madani \\ University, Tabriz, Iran \\ Full list of author information is \\ available at the end of the article
}

\begin{abstract}
This research is conducted for studying some qualitative specifications of solution to a generalized fractional structure of the standard snap boundary problem. We first rewrite the mathematical model of the extended fractional snap problem by means of the $\mathbb{G}$-operators. After finding its equivalent solution as a form of the integral equation, we establish the existence criterion of this reformulated model with respect to some known fixed point techniques. Then we analyze its stability and further investigate the inclusion version of the problem with the help of some special contractions. We present numerical simulations for solutions of several examples regarding the fractional $\mathbb{G}$-snap system in different structures including the Caputo, Caputo-Hadamard, and Katugampola operators of different orders.
\end{abstract}

MSC: $34 \mathrm{~A} 08 ; 34 \mathrm{~A} 12$

Keywords: Fixed-point; Generalized fractional operators; Snap equation; Special operators

\section{Introduction}

Fractional calculus is one of the most important branches of applied mathematics. The main importance of this field can be observed in many published papers regarding different fractional differential equations and inclusions in recent years. In this direction, different generalizations of derivatives have been introduced by some researchers. For example, recently, Lazreg et al. [1] investigated the Cauchy problem of Caputo-Fabrizio impulsive fractional differential equations

$$
\left\{\begin{array}{l}
\left({ }^{\mathrm{CF}} \mathcal{D}_{a_{k}}^{r} \mathrm{v}\right)(\mathfrak{t})=f(\mathrm{t}, \mathrm{v}(\mathfrak{t})), \quad \mathfrak{t} \in \mathbb{I}_{k}, k=0,1, \ldots, \mathrm{m}, \\
\mathrm{v}\left(a_{k}^{+}\right)=\mathrm{v}\left(a_{k}^{-}\right)+\varrho_{k}\left(\mathrm{v}\left(a_{k}^{-}\right)\right), \quad k=1,2, \ldots, \mathrm{m}, \\
\mathrm{v}(0)=\mathrm{v}_{0}
\end{array}\right.
$$

where $\mathbb{I}_{0}=\left[0, a_{1}\right], \mathbb{I}_{k}=\left(a_{k}, a_{k+1}\right], k=1,2, \ldots, \mathrm{m}, 0=a_{0}<a_{1}<a_{2}<\cdots<a_{\mathrm{m}}<a_{\mathrm{m}+1}=\tau, \mathrm{v}_{0} \in$ $\mathbb{R}, f: \mathbb{I}_{k} \times \mathbb{R} \rightarrow \mathbb{R}(k=0,1, \ldots, \mathrm{m})$ and $\varrho_{k}: \mathbb{R} \rightarrow \mathbb{R}(k=1, \ldots, \mathrm{m})$ are given continuous functions, and ${ }^{\mathrm{CF}} \mathcal{D}_{a_{k}}^{r}$ is the Caputo-Fabrizio derivative of order $r \in(0,1)$. Also, Krim et al.

(c) The Author(s) 2021. This article is licensed under a Creative Commons Attribution 4.0 International License, which permits use, sharing, adaptation, distribution and reproduction in any medium or format, as long as you give appropriate credit to the original author(s) and the source, provide a link to the Creative Commons licence, and indicate if changes were made. The images or other third party material in this article are included in the article's Creative Commons licence, unless indicated otherwise in a credit line to the material. If material is not included in the article's Creative Commons licence and your intended use is not permitted by statutory regulation or exceeds the permitted use, you will need to obtain permission directly from the copyright holder. To view a copy of this licence, visit http://creativecommons.org/licenses/by/4.0/. 
[2] considered the class of terminal value problems of Katugampola implicit differential equations of noninteger orders

$$
\left\{\begin{array}{l}
\left({ }^{K} \mathcal{D}_{0^{+}}^{r}+\mathrm{v}\right)(\mathfrak{t})=f\left(\mathfrak{t}, \mathrm{v}(\mathfrak{t}),\left({ }^{K} \mathcal{D}_{0^{+}}^{r}+\mathrm{v}\right)(\mathfrak{t})\right), \quad \mathbb{I}=\left[0, \tau_{0}\right], \\
\mathrm{v}\left(\tau_{0}\right)=\mathrm{v}_{0} \in \mathbb{R}, \quad \tau>0,
\end{array}\right.
$$

where the function $f: \mathbb{I} \times \mathbb{R}^{2} \rightarrow \mathbb{R}$ is continuous, and ${ }^{K} \mathcal{D}_{0^{+}}^{r}$ is the Katugampola fractional derivative of order $r \in(0,1]$. In 2020, Baitiche et al. [3] generalized the fractional settings and studied the existence of solutions of the following $\psi$-Caputo fractional differential equation:

$$
\left\{\begin{array}{l}
{ }^{C} \mathcal{D}_{a^{+}}^{q, \psi} \mathrm{v}(\mathfrak{t})+f(\mathfrak{t}, \mathrm{v}(\mathfrak{t}))=0, \quad \mathfrak{t} \in \mathbb{J}=[a, b], \\
\mathrm{v}(a)=\mathrm{v}^{\prime}(a)=0, \quad \mathrm{v}(b)=\sum_{i=1}^{m} \lambda_{i} \mathrm{v}\left(\eta_{i}\right), \quad \eta_{i} \in(a, b),
\end{array}\right.
$$

where ${ }^{C} \mathcal{D}_{a^{+}}^{q, \psi}$ is the $\psi$-Caputo fractional derivative of order $q \in(2,3], \mathrm{w}: \mathbb{J} \times \mathbb{R} \rightarrow \mathbb{R}$ is a given continuous function, and $\lambda_{i}$ are real constants satisfying $\Delta=\sum_{i=1}^{m} \lambda_{i}\left(\psi\left(\eta_{i}\right)-\psi(a)\right)^{2}-$ $(\psi(b)-\psi(a))^{2} \neq 0$. Also, Wahash et al. [4] investigated the existence and interval of existence, uniqueness, estimates of solutions, and different types of Ulam stability results of solutions on a subinterval of $[0, b]$ for the nonlinear fractional differential equation involving generalized Caputo fractional derivatives with respect to the function $\psi$ given by ${ }^{C} \mathcal{D}_{a^{+}}^{q, \psi} \mathrm{v}(\mathfrak{t})=f(\mathfrak{t}, \mathrm{v}(\mathfrak{t})), \mathfrak{t} \in[0, b]$, with nonlocal condition $\mathrm{v}(0)=\hbar(\mathrm{v})=\mathrm{v}_{0}$, where $q \in(0,1)$, $\mathrm{v}_{0} \in \mathbb{R},{ }^{C} \mathcal{D}_{a^{+}}^{q, \psi}$ denotes the $\psi$-Caputo fractional derivative of order $q, f:[0, b] \times \mathbb{R} \rightarrow \mathbb{R}$ and $\hbar: C([0, b], \mathbb{R}) \rightarrow \mathbb{R}$ are nonlinear continuous functions, and $\mathrm{v} \in C([0, b], \mathbb{R})$ is such that the operator ${ }^{C} \mathcal{D}_{a^{+}}^{q, \psi}$ exists and ${ }^{C} \mathcal{D}_{a^{+}}^{q, \psi} \in C([0, b], \mathbb{R})$.

In 2019, Pham et al. [5] introduced a chaotic integer-order system, called a snap system, which involves only one quadratic nonlinear term and takes the following mathematical form:

$$
\left\{\begin{array}{l}
\frac{d v_{1}}{d t}=v_{2}(t) \\
\frac{d v_{2}}{d t}=v_{3}(t), \\
\frac{d v_{3}}{d t}=v_{4}(t), \\
\frac{d v_{4}}{d t}=\mathcal{T}\left(v_{1}, v_{2}, v_{3}, v_{4}\right),
\end{array}\right.
$$

where $\mathcal{T}\left(\mathrm{v}_{1}, \mathrm{v}_{2}, \mathrm{v}_{3}, \mathrm{v}_{4}\right)=-a \mathrm{v}_{1}-\mathrm{v}_{2}-\mathrm{v}_{4}+b \mathrm{v}_{1} \mathrm{v}_{3}$. Equation (1) can be transformed into a fourth-order differential equation

$$
\frac{\mathrm{d}^{4} \mathrm{v}_{1}}{\mathrm{d \textrm {t } ^ { 4 }}}=\mathcal{T}\left(\mathrm{v}_{1}, \frac{\mathrm{dv_{1 }}}{\mathrm{dt}}, \frac{\mathrm{d}^{2} \mathrm{v}_{1}}{\mathrm{dt}^{2}}, \frac{\mathrm{d}^{3} \mathrm{v}_{1}}{\mathrm{dt}^{3}}\right)
$$

The new equation (2) contains a fourth-order derivative of the variable $\mathrm{v}_{1}$, which in physics stands for a second derivative of acceleration in a mechanical system. Equation (2) is called a snap or jounce equation and describes a fourth-order dynamical model.

Many researchers have investigated sufficient conditions for the uniqueness, existence, stability, and attractivity of solutions for a wide domain of fractional nonlinear ordinary differential equations (ODEs) or mathematical models containing different fractional 
derivatives by using numerous types of methods including standard fixed point theory, Tdegree theory, variational methods, monotone iterative approaches, MNC technique, and so on. For more detail, see [6-23]. However, to the best of our knowledge, limited results can be found on the existence and stability of solutions of fractional snap systems via the generalized $\mathbb{G}$-Caputo derivative.

The authors in [24] studied the fractional snap model

$$
\left\{\begin{array}{l}
{ }^{c} \mathcal{D}^{q} \mathrm{v}_{1}=\mathrm{v}_{2}(\mathfrak{t}) \\
{ }^{c} \mathcal{D}^{q} \mathrm{v}_{2}=\mathrm{v}_{3}(\mathfrak{t}) \\
{ }^{c} \mathcal{D}^{q} \mathrm{v}_{3}=\mathrm{v}_{4}(\mathfrak{t}) \\
{ }^{c} \mathcal{D}^{q} \mathrm{v}_{4}=-a \mathrm{v}_{1}-\mathrm{v}_{2}-\mathrm{v}_{4}+b \mathrm{v}_{1} \mathrm{v}_{3}
\end{array}\right.
$$

where $a=2, b=1$, and the Caputo fractional order $q=0.95$.

In view of the above facts, in this paper, we focus our attention on the problem of the existence and uniqueness along with the Hyers-Ulam stability of solutions for different forms of fractional nonlinear snap systems in the $\mathbb{G}$-Caputo sense with initial conditions. Namely, we study the following problem:

$$
\left\{\begin{array}{l}
{ }^{c} \mathcal{D}_{a^{+}}^{q ; \mathfrak{G}} \mathrm{v}(\mathfrak{t})=\mathrm{u}(\mathfrak{t}), \quad \mathrm{v}(a)=\mathrm{v}_{0} \\
{ }^{c} \mathcal{D}_{a^{+}}^{p: \mathbb{G}} \mathrm{u}(\mathfrak{t})=\mathrm{w}(\mathfrak{t}), \quad \mathrm{u}(a)=\mathrm{v}_{1} \\
{ }^{c} \mathcal{D}_{a^{+}}^{r ; \mathfrak{G}} \mathrm{w}(\mathfrak{t})=\mathrm{x}(\mathfrak{t}), \quad \mathrm{w}(a)=\mathrm{v}_{2}, \\
{ }^{c} \mathcal{D}_{a^{+}}^{k: \mathbb{G}} \mathrm{x}(\mathfrak{t})=h(\mathfrak{t}, \mathrm{v}, \mathrm{u}, \mathrm{w}, \mathrm{x}), \quad \mathrm{x}(a)=\mathrm{v}_{3}
\end{array}\right.
$$

where ${ }^{c} \mathcal{D}_{a^{+}}^{\eta ; \mathbb{G}}$ are the $\mathbb{G}$-Caputo derivatives, $\eta$ belong to $\{q, p, r, k\}$ such that $0<q, p, r, k \leq 1$, the increasing function $\mathbb{G} \in C^{1}([a, b])$ is such that $\mathbb{G}^{\prime}(\mathfrak{t}) \neq 0, \mathfrak{t} \in[a, b], h \in C\left([a, b] \times \mathbb{R}^{4}, \mathbb{R}\right)$, and $\mathrm{v}_{0}, \mathrm{v}_{1}, \mathrm{v}_{2}, \mathrm{v}_{3} \in \mathbb{R}$. It is obvious that this system can be rewritten as

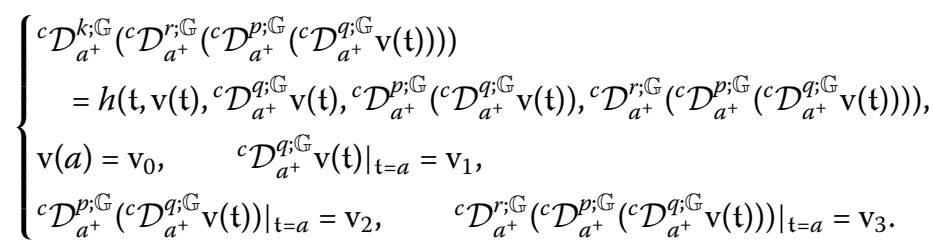

It is natural that if we set $\mathbb{G}(\mathfrak{t})=\mathfrak{t}, a=0$, and $q=p=r=k=1$, then we obtain the standard 4th-order ODE (2) with initial conditions. Our method in this paper is based on fixed point approaches. Also, we can find more ideas on fractional calculus and its applications in $[3,25-41]$.

The summary of our work in this research is as follows. In Sect. 2, we recall several assembled concepts of fractional calculus, useful lemmas, and some theorems about the fixed points. In Sect. 3, we give the proof of the fundamental theorems of this paper by utilizing fixed point approaches such as Banach's principle and Schauder's theorem. In Sect. 4, we discuss the stability in the context of the Ulam-Hyers stability, its generalized version along with Ulam-Hyers-Rassias stability, and its generalized version for solutions of the fractional $\mathbb{G}$-snap system (4). In Sect. 5, we utilize a special form of contractions to prove the existence results for an inclusion version of (4). Appropriate applications with 
numerical simulation are provided in Sect. 6 to illustrate and analyze the obtained results. Finally, in Sect. 7, we give the conclusion of our article.

\section{Preliminaries}

Here we recall some initial notions, definitions and notations.

Let $\mathbb{G}:[a, b] \rightarrow \mathbb{R}$ be increasing via $\mathbb{G}^{\prime}(\mathfrak{t}) \neq 0$ for all $\mathfrak{t}$. We start this part by defining the $\mathbb{G}$-Riemann-Liouville fractional ( $\mathbb{G}-\mathrm{FRL}$ ) integrals and derivatives. In this section, we set

$$
A=\left(\frac{1}{\mathbb{G}^{\prime}(\mathfrak{t})} \frac{\mathrm{d}}{\mathrm{d} \mathfrak{t}}\right)
$$

Definition 2.1 $([42,43])$ For $\eta>0$, the $\eta$ th $\mathbb{G}$-FRL integral of an integrable function v : $[a, b] \rightarrow \mathbb{R}$ with respect to $\mathbb{G}$ is given as follows:

$$
\mathcal{I}_{a^{+}}^{\eta ; \mathbb{G}} \mathbf{v}(\mathfrak{t})=\frac{1}{\Gamma(\eta)} \int_{a}^{\mathfrak{t}}(\mathbb{G}(\mathfrak{t})-\mathbb{G}(\xi))^{\eta-1} \mathbb{G}^{\prime}(\xi) \mathrm{v}(\xi) \mathrm{d} \xi
$$

where $\Gamma(\eta)=\int_{0}^{+\infty} e^{-\mathfrak{t}} \mathfrak{t}^{\eta-1} \mathrm{~d} \mathfrak{t}, \eta>0$.

Let $n \in \mathbb{N}$, and let $\mathbb{G}, \mathrm{v} \in C^{n}([a, b], \mathbb{R})$ be such that $\mathbb{G}$ has the same properties mentioned above. The $\eta$ th $\mathbb{G}$-FRL derivative of $\mathrm{v}$ is defined by

$$
\begin{aligned}
\mathcal{D}_{a^{+}}^{\eta ; \mathbb{G}} \mathrm{v}(\mathfrak{t}) & =A^{(n)} \mathcal{I}_{a^{+}}^{n-\eta ; \mathbb{G}} \mathrm{v}(\mathfrak{t}) \\
& =\frac{1}{\Gamma(n-\eta)} A^{(n)} \int_{a}^{\mathfrak{t}}(\mathbb{G}(\mathfrak{t})-\mathbb{G}(\xi))^{n-\eta-1} \mathbb{G}^{\prime}(\xi) \mathrm{v}(\xi) \mathrm{d} \xi,
\end{aligned}
$$

where $n=[\eta]+1[42,43]$. The $\eta$ th $\mathbb{G}$-fractional Caputo derivative of $\mathrm{v}$ is defined by ${ }^{c} \mathcal{D}_{a^{+}}^{\eta ; \mathbb{G}} \mathrm{v}(\mathfrak{t})=\mathcal{I}_{a^{+}}^{n-\eta ; \mathbb{G}} A^{(n)} \mathrm{v}(\mathfrak{t})$, where $n=[\eta]+1$ for $\eta \notin \mathbb{N}$ and $n=\eta$ for $\eta \in \mathbb{N}$ [44]. In other words,

$$
{ }^{c} \mathcal{D}_{a^{+}}^{\eta ; \mathbb{G}} \mathbf{v}(\mathfrak{t})= \begin{cases}\int_{a}^{\mathfrak{t}} \frac{(\mathbb{G}(\mathfrak{t})-\mathbb{G}(\xi))^{n-\eta-1}}{\Gamma(n-\eta)} \mathbb{G}^{\prime}(\xi) A^{(n)} \mathrm{v}(\xi) \mathrm{d} \xi, & \eta \notin \mathbb{N}, \\ A^{n} \mathrm{v}(\mathfrak{t}), & \eta=n \in \mathbb{N} .\end{cases}
$$

Extension (6) gives the Caputo derivative when $\mathbb{G}(\mathfrak{t})=\mathfrak{t}$. Also, in the case $\mathbb{G}(\mathfrak{t})=\ln \mathfrak{t}$, it yields the Caputo-Hadamard derivative. If $\mathrm{v} \in C^{n}([a, b], \mathbb{R})$, then the $\eta$ th $\mathbb{G}$-fractional Caputo derivative of $\mathrm{v}$ is specified as [44, Theorem 3]

$$
{ }^{c} \mathcal{D}_{a^{+}}^{\eta ; \mathbb{G}} \mathrm{v}(\mathfrak{t})=\mathcal{D}_{a^{+}}^{\eta ; \mathbb{G}}\left(\mathrm{v}(\mathfrak{t})-\sum_{j=0}^{n-1} \frac{A^{(j)} \mathrm{v}(a)}{j !}(\mathbb{G}(\mathfrak{t})-\mathbb{G}(a))^{j}\right) .
$$

The composition rules for the above $\mathbb{G}$-operators are recalled in the following lemma.

Lemma 2.2 ([45]) Let $n-1<\eta<n$ and $\mathrm{v} \in C^{n}([a, b], \mathbb{R})$. Then

$$
\mathcal{I}_{a^{+}}^{\eta ; \mathbb{G}_{c}} \mathcal{D}_{a^{+}}^{\eta ; \mathbb{G}} \mathrm{v}(\mathfrak{t})=\mathrm{v}(\mathfrak{t})-\sum_{j=0}^{n-1} \frac{A^{(j)} \mathrm{v}(a)}{j !}[\mathbb{G}(\mathfrak{t})-\mathbb{G}(a)]^{j}
$$


for all $\mathfrak{t} \in[a, b]$. Moreover, if $m \in \mathbb{N}$ and $\mathrm{v} \in C^{n+m}([a, b], \mathbb{R})$, then

$$
A^{(m)}\left({ }^{c} \mathcal{D}_{a^{+}}^{\eta ; \mathbb{G}} \mathrm{v}\right)(\mathfrak{t})={ }^{c} \mathcal{D}_{a^{+}}^{\eta+m ; \mathbb{G}} \mathbf{v}(\mathfrak{t})+\sum_{j=0}^{m-1} \frac{[\mathbb{G}(\mathfrak{t})-\mathbb{G}(a)]^{j+n-\eta-m}}{\Gamma(j+n-\eta-m+1)} A^{(j+n)} \mathrm{v}(a)
$$

From equation (7) observe that if $A^{(j)} \mathrm{v}(a)=0$ for $j=n, n+1, \ldots, n+m-1$, then $A^{(m)}\left({ }^{c} \mathcal{D}_{a^{+}}^{\eta ; \mathbb{G}} \mathrm{v}\right)(\mathfrak{t})={ }^{c} \mathcal{D}_{a^{+}}^{\eta+m ; \mathbb{G}} \mathrm{v}(\mathfrak{t}), \mathfrak{t} \in[a, b]$.

Lemma $2.3([45])$ Let $\eta, v>0$ and $\mathrm{v} \in C([a, b], \mathbb{R})$. Then for all $\mathfrak{t} \in[a, b]$, denoting $F_{a}(\mathfrak{t})=$ $\mathbb{G}(\mathfrak{t})-\mathbb{G}(a)$, we have

1. $\mathcal{I}_{a^{+}}^{\eta ; \mathbb{G}}\left(\mathcal{I}_{a^{+}}^{v ; \mathbb{G}} \mathrm{v}\right)(\mathfrak{t})=\mathcal{I}_{a^{+}}^{\eta+v ; \mathbb{G}} \mathrm{v}(\mathfrak{t})$

2. ${ }^{c} \mathcal{D}_{a^{+}}^{\eta ; \mathbb{G}^{+}}\left(\mathcal{I}_{a^{+}}^{\eta ; \mathbb{G}} \mathrm{v}\right)(\mathfrak{t})=\mathrm{v}(\mathfrak{t})$,

3. $\mathcal{I}_{a^{+}}^{\eta ; \mathbb{G}}\left(F_{a}(\mathfrak{t})\right)^{\nu-1}=\frac{\Gamma(v)}{\Gamma(v+\eta)}\left(F_{a}(\mathfrak{t})\right)^{v+\eta-1}$,

4. ${ }^{c} \mathcal{D}_{a^{+}}^{\eta ; \mathbb{G}}\left(F_{a}(\mathfrak{t})\right)^{\nu-1}=\frac{\Gamma(\nu)}{\Gamma(\nu-\eta)}\left(F_{a}(\mathfrak{t})\right)^{\nu-\eta-1}$,

5. ${ }^{c} \mathcal{D}_{a^{+}}^{\eta ; \mathbb{G}}\left(F_{a}(\mathfrak{t})\right)^{j}=0,(j=0,1, \ldots, n-1), n \in \mathbb{N}, n-1 \leq \eta \leq n$.

To end this part of the paper, we state the following fixed point theorems.

Theorem 2.4 (Banach contraction principle [46]) Let $(\mathbb{V}, \rho)$ be a nonempty complete metric space, and let $\Psi: \mathbb{V} \rightarrow \mathbb{V}$ be a contraction, that is,

$$
\rho\left(\Psi \mathrm{v}, \Psi \mathrm{v}^{*}\right) \leq \mu \rho\left(\mathrm{v}, \mathrm{v}^{*}\right) \quad \text { for all } \mathrm{v}, \mathrm{v}^{*} \in \mathbb{V}
$$

and for some $\mu \in(0,1)$. Then $\Psi$ admits a unique fixed point.

Theorem 2.5 (Leray-Schauder [46]) Let $\mathbb{V}$ be a Banach space, let $\Sigma$ be a bounded convex closed subset of $\mathbb{V}$, and let $\mathbb{U}$ be an open set contained in $\Sigma$ with $0 \in \mathbb{U}$. Let $\Psi: \overline{\mathbb{U}} \rightarrow \Sigma$ be a continuous and compact mapping. Then either (i) $\Psi$ admits a fixed point belonging to $\overline{\mathbb{U}}$, or (ii) there exist $\mathrm{v} \in \partial \mathbb{U}$ and $\mu \in(0,1)$ such that $\mathrm{v}=\mu \Psi(\mathrm{v})$.

Consider normed space $(\mathcal{C},\|\cdot\|)$. The collection of all closed, bounded, compact and convex subsets of $\mathcal{C}$ are denoted by $\mathcal{P}_{\mathrm{CL}}(\mathcal{C}), \mathcal{P}_{\mathrm{BN}}(\mathcal{C}), \mathcal{P}_{\mathrm{CP}}(\mathcal{C})$, and $\mathcal{P}_{\mathrm{CV}}(\mathcal{C})$, respectively.

Definition 2.6 ([47]) Consider $v: \mathbb{R} \rightarrow \mathbb{R}$ as a real-valued function and $\mathfrak{H}$ as a multifunction. (i) $\mathfrak{H}$ is u.s.c on $\mathcal{C}$ if $\mathfrak{H}\left(\mathrm{v}^{*}\right) \in \mathcal{P}_{\mathrm{CL}}(\mathcal{C})$ for any $\mathrm{v}^{*} \in \mathcal{C}$, and also there exists a neighborhood $\mathfrak{N}_{0}^{*}$ of $\mathrm{v}^{*}$ subject to $\mathfrak{H}\left(\mathfrak{N}_{0}^{*}\right) \subseteq \mathbb{O}$ for $\mathbb{O} \subseteq \mathcal{C}$, where $\mathbb{O}$ is an arbitrary open set. (ii) A

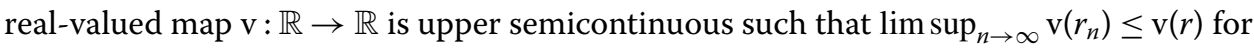
each $\left\{r_{n}\right\}_{n \geq 1}$ with $r_{n} \rightarrow r$.

A Pompeiu-Hausdorff metric $\mathcal{H}_{\rho}:(\mathcal{P}(\mathcal{C}))^{2} \rightarrow \mathbb{R} \cup\{\infty\}$ is defined as

$$
\mathcal{H}_{\rho}\left(\mathcal{A}_{1}^{*}, \mathcal{A}_{2}^{*}\right)=\max \left\{\sup _{a_{1}^{*} \in \mathcal{A}_{1}^{*}} \rho\left(a_{1}^{*}, \mathcal{A}_{2}^{*}\right), \sup _{A_{2}^{*} \in \mathcal{A}_{2}^{*}} \rho\left(\mathcal{A}_{1}^{*}, a_{2}^{*}\right)\right\},
$$

where $\rho$ is the metric of $\mathcal{M}$, and [47] $\rho\left(\mathcal{A}_{1}^{*}, a_{2}^{*}\right)=\inf _{a_{1}^{*} \in \mathcal{A}_{1}^{*}} \rho\left(a_{1}^{*}, a_{2}^{*}\right)$ and $\rho\left(a_{1}^{*}, \mathcal{A}_{2}^{*}\right)=$ $\inf _{A_{2}^{*} \in \mathcal{A}_{2}^{*}} \rho\left(a_{1}^{*}, a_{2}^{*}\right)$. Suppose for $\mathfrak{H}: \mathcal{C} \rightarrow \mathcal{P}_{\mathrm{CL}}(\mathcal{C})$ and $\mathrm{v}_{1}, \mathrm{v}_{2} \in \mathcal{M}$, we have the inequality

$$
\mathcal{H}_{\rho}\left(\mathfrak{H}\left(\mathrm{v}_{1}\right), \mathfrak{H}\left(\mathrm{v}_{2}\right)\right) \leq L \rho\left(\mathrm{v}_{1}, \mathrm{v}_{2}\right)
$$


Then $\mathfrak{H}$ is said to be (H1) a Lipschitz map if $L>0$ and (H2) a contraction if $0<L<1$ [47].

Definition 2.7 ([47]) (i) $\mathfrak{H}:[a, b] \times \mathbb{R} \rightarrow \mathcal{P}(\mathbb{R})$ is Carathéodory if $\mathfrak{t} \mapsto \mathfrak{H}(\mathfrak{t}, \mathrm{v})$ is measurable for any $\mathrm{v} \in \mathbb{R}$ and $\mathrm{v} \mapsto \mathfrak{H}(\mathfrak{t}, \mathrm{v})$ is u.s.c for a.e. $\mathfrak{t} \in[a, b]$. (ii) A Carathéodory multifunction $\mathfrak{H}:[a, b] \times \mathbb{R} \rightarrow \mathcal{P}(\mathbb{R})$ is $L^{1}$-Carathéodory if for any $\epsilon>0$, there exists $\kappa_{\epsilon} \in L^{1}\left([a, b], \mathbb{R}_{+}\right)$ such that

$$
\|\mathfrak{H}(\mathfrak{t}, \mathrm{v})\|=\sup _{\mathfrak{t} \in[a, b]}\{|\omega|: \omega \in \mathfrak{H}(\mathfrak{t}, \mathrm{v})\} \leq \kappa_{\epsilon}(\mathfrak{t})
$$

for all $|v| \leq \epsilon$ and almost all $\mathfrak{t} \in[a, b]$.

Definition 2.8 ([48]) Let $\psi: \mathbb{R}_{\geq 0} \rightarrow \mathbb{R}_{\geq 0}$ be a nondecreasing map belonging to class $\Pi$ such that for all $\mathfrak{t}>0, \sum_{j=1}^{\infty} \psi^{j}(\mathfrak{t})<\infty$ and $\psi(\mathfrak{t})<\mathfrak{t}$. Let $\Phi^{*}: \mathcal{C} \rightarrow \mathcal{C}$ and $\alpha: \mathcal{C}^{2} \rightarrow \mathbb{R}_{\geq 0}$. Then

(i) $\Phi^{*}$ is $\alpha-\psi$-contraction if for $\mathrm{v}_{1}, \mathrm{v}_{2} \in \mathcal{C}$,

$$
\alpha\left(\mathrm{v}_{1}, \mathrm{v}_{2}\right) \rho\left(\Phi^{*} \mathrm{v}_{1}, \Phi^{*} \mathrm{v}_{2}\right) \leq \psi\left(\rho\left(\mathrm{v}_{1}, \mathrm{v}_{2}\right)\right) .
$$

(ii) $\Phi^{*}$ is $\alpha$-admissible if $\alpha\left(\mathrm{v}_{1}, \mathrm{v}_{2}\right) \geq 1$ gives $\alpha\left(\Phi^{*} \mathrm{v}_{1}, \Phi^{*} \mathrm{v}_{2}\right) \geq 1$.

(iii) $\mathcal{C}$ has property (B) if for every sequence $\left\{\mathrm{v}_{n}\right\}_{n \geq 1}$ of $\mathcal{C}$ with $\alpha\left(\mathrm{v}_{n}, \mathrm{v}_{n+1}\right) \geq 1$ and $\mathrm{v}_{n} \rightarrow \mathrm{v}$, we have $\alpha\left(\mathrm{v}_{n}, \mathrm{v}\right) \geq 1$ for all $n \geq 1$.

Definition 2.9 ([49]) Let $\psi: \mathbb{R}_{\geq 0} \rightarrow \mathbb{R}_{\geq 0}$ be a nondecreasing map belonging to class $\Pi$ such that for all $\mathfrak{t}>0, \sum_{j=1}^{\infty} \psi^{j}(\mathfrak{t})<\infty$ and $\psi(\mathfrak{t})<\mathfrak{t}$. Let $\mathfrak{H}: \mathcal{C} \rightarrow \mathcal{P}(\mathcal{C})$ and $\alpha: \mathcal{C}^{2} \rightarrow \mathbb{R}_{\geq 0}$. Then

(i) $\mathfrak{H}: \mathcal{C} \rightarrow \mathcal{P}_{\mathrm{CL}, \mathrm{BN}}(\mathcal{C})$ is $\alpha-\psi$-contraction if for all $\mathrm{v}_{1}, \mathrm{v}_{2} \in \mathcal{C}$,

$$
\alpha\left(\mathrm{v}_{1}, \mathrm{v}_{2}\right) \mathcal{H}_{\rho}\left(\mathfrak{H}_{\mathrm{v}_{1}}, \mathfrak{H} \mathrm{v}_{2}\right) \leq \psi\left(\rho\left(\mathrm{v}_{1}, \mathrm{v}_{2}\right)\right)
$$

(ii) $\mathfrak{H}$ is $\alpha$-admissible if for all $\mathrm{v}_{1} \in \mathcal{C}$ and $\mathrm{v}_{2} \in \mathfrak{H} \mathrm{v}_{1}$, the inequality $\alpha\left(\mathrm{v}_{1}, \mathrm{v}_{2}\right) \geq 1$ gives $\alpha\left(\mathrm{v}_{2}, \mathrm{v}_{3}\right) \geq 1$ for each $\mathrm{v}_{3} \in \mathfrak{H v}_{2}$.

(iii) $\mathcal{C}$ has property $\left(C_{\alpha}\right)$ if for every sequence $\left\{\mathrm{v}_{n}\right\}_{n \geq 1}$ of $\mathcal{C}$ with $\mathrm{v}_{n} \rightarrow \mathrm{v}$ and $\alpha\left(\mathrm{v}_{n}, \mathrm{v}_{n+1}\right) \geq 1$, there exists a subsequence $\left\{\mathrm{v}_{n_{k}}\right\}$ of $\left\{\mathrm{v}_{n}\right\}$ such that $\alpha\left(\mathrm{v}_{n_{k}}, \mathrm{v}\right) \geq 1$ for all $k \in \mathbb{N}$.

Theorem 2.10 ([48]) Let $(\mathcal{C}, \rho)$ a complete metric space, and let $\psi \in \Pi, \alpha: \mathcal{C}^{2} \rightarrow \mathbb{R}$, and $\Phi^{*}: \mathcal{C} \rightarrow \mathcal{C}$. Assume that: (i) $\Phi^{*}$ is $\alpha$-admissible and $\alpha$ - $\psi$-contraction, (ii) $\alpha\left(\mathrm{v}_{0}, \Phi^{*} \mathrm{v}_{0}\right) \geq 1$ for some $\mathrm{v}_{0} \in \mathcal{C}$, and (iii) $\mathcal{C}$ has property $(B)$. Then $\Phi^{*}$ has a fixed point.

Theorem 2.11 ([50]) Let $\mathcal{C}$ be a Banach space, and let $\mathbb{A} \neq \emptyset$ belong to $\mathcal{P}_{\mathrm{CL}, \mathrm{BN}, \mathrm{CV}}(\mathcal{C})$. Suppose that for $\mathfrak{T}_{1}$ and $\mathfrak{T}_{1}$ defined on $\mathbb{A},(i) \mathfrak{T}_{1} \mathrm{v}+\mathfrak{T}_{2} \mathrm{v}^{\prime} \in \mathbb{A}$ for $\mathrm{v}, \mathrm{v}^{\prime} \in \mathbb{A}$, (ii) $\mathfrak{T}_{1}$ is compactcontinuous, and (iii) $\mathfrak{T}_{2}$ is a contraction. Then there exists $\mathrm{v}_{*} \in \mathbb{A}$ such that $\mathrm{v}_{*}=\mathfrak{T}_{1} \mathrm{v}_{*}+\mathfrak{T}_{2} \mathrm{v}_{*}$.

Theorem 2.12 ([49]) Let $(\mathcal{C}, \rho)$ be a complete metric space, and let $\psi \in \Pi, \alpha: \mathcal{C}^{2} \rightarrow$ $\mathbb{R}_{\geq 0}$, and $\mathfrak{H}: \mathcal{C} \rightarrow \mathcal{P}_{\mathrm{CL}, \mathrm{BN}}(\mathcal{C})$. Assume that $(i) \mathfrak{H}$ is an $\alpha$-admissible $\alpha$ - $\psi$-contraction, (ii) $\alpha\left(\mathrm{v}_{0}, \mathrm{v}_{1}\right) \geq 1$ for some $\mathrm{v}_{0} \in \mathcal{C}$ and $\mathrm{v}_{1} \in \mathfrak{H} \mathrm{v}_{0}$, and (iii) $\mathcal{C}$ has property $\left(C_{\alpha}\right)$. Then $\mathfrak{H}$ has a fixed point. 
Theorem 2.13 ([47]) Let $(\mathcal{C}, \rho)$ be a complete metric space. Assume that $(i) \psi \in \Pi$ is u.s.c such that $\liminf _{\mathfrak{t} \rightarrow \infty}(\mathfrak{t}-\psi(\mathfrak{t}))>0$ for $\mathfrak{t}>0$ and $($ ii $) \mathfrak{H}: \mathcal{C} \rightarrow \mathcal{P}_{\mathrm{CL}, \mathrm{BN}}(\mathcal{C})$ satisfies the property

$$
\mathcal{H}_{\rho}\left(\mathfrak{H t}_{1}, \mathfrak{H t}_{2}\right) \leq \psi\left(\rho\left(\mathfrak{t}_{1}, \mathfrak{t}_{2}\right)\right), \quad \mathfrak{t}_{1}, \mathfrak{t}_{2} \in \mathcal{C}
$$

Then $\mathfrak{H}$ has a unique end-point iff $\mathfrak{H}$ has the $(A E P)$-property.

\section{Existence and uniqueness results}

Here we analyze the existence properties of solutions and their uniqueness for the proposed fractional $\mathbb{G}$-snap problem (4). We need the following lemma, which specifies the corresponding integral equation.

Lemma 3.1 Let $q, p, r, k \in(0,1]$ and $\mathrm{v}_{0}, \mathrm{v}_{1}, \mathrm{v}_{2}, \mathrm{v}_{3} \in \mathbb{R}$. If $g \in C([a, b], \mathbb{R})$, then the linear $\mathbb{G}$ snap FBVP

$$
\left\{\begin{array}{l}
{ }^{c} \mathcal{D}_{a^{+}}^{k ; \mathbb{G}}\left({ }^{c} \mathcal{D}_{a^{+}}^{r ; \mathbb{G}}\left({ }^{c} \mathcal{D}_{a^{+}}^{p ; \mathbb{G}}\left({ }^{c} \mathcal{D}_{a^{+}}^{q ; \mathbb{G}} \mathrm{v}(\mathfrak{t})\right)\right)\right)=g(\mathfrak{t}), \\
\mathrm{v}(a)=\mathrm{v}_{0}, \quad{ }^{c} \mathcal{D}_{a^{+}}^{q ; \mathbb{G}} \mathrm{v}(a)=\mathrm{v}_{1}, \\
{ }^{c} \mathcal{D}_{a^{+}}^{p ; \mathbb{G}}\left({ }^{c} \mathcal{D}_{a^{+}}^{q ; \mathbb{G}} \mathrm{v}(a)\right)=\mathrm{v}_{2}, \quad{ }^{c} \mathcal{D}_{a^{+}}^{r ; \mathbb{G}}\left({ }^{c} \mathcal{D}_{a^{+}}^{p ; \mathbb{G}}\left({ }^{c} \mathcal{D}_{a^{+}}^{q ; \mathbb{G}} \mathrm{v}(a)\right)\right)=\mathrm{v}_{3}
\end{array}\right.
$$

has the solution

$$
\begin{aligned}
\mathrm{v}(\mathfrak{t})= & \mathrm{v}_{0}+\frac{\mathrm{v}_{1}(\mathbb{G}(\mathfrak{t})-\mathbb{G}(a))^{q}}{\Gamma(q+1)}+\frac{\mathrm{v}_{2}(\mathbb{G}(\mathfrak{t})-\mathbb{G}(a))^{q+p}}{\Gamma(q+p+1)} \\
& +\frac{\mathrm{v}_{3}(\mathbb{G}(\mathfrak{t})-\mathbb{G}(a))^{q+p+r}}{\Gamma(q+p+r+1)} \\
& +\int_{a}^{\mathfrak{t}} \mathbb{G}^{\prime}(\xi) \frac{(\mathbb{G}(\mathfrak{t})-\mathbb{G}(\xi))^{q+p+r+k-1}}{\Gamma(q+p+r+k)} g(\xi) \mathrm{d} \xi .
\end{aligned}
$$

Proof Consider $v(\mathfrak{t})$ satisfying the linear fractional $\mathbb{G}$-snap problem (3.1). Applying the $k$ th $\mathbb{G}$-integral operator $\mathcal{I}_{a^{+}}^{k ; \mathbb{G}}$ to both sides of equation (8), by the 4 th boundary condition we obtain

$$
\begin{aligned}
{ }^{c} \mathcal{D}_{a^{+}}^{r ; \mathbb{G}}\left({ }^{c} \mathcal{D}_{a^{+}}^{p ; \mathbb{G}}\left({ }^{c} \mathcal{D}_{a^{+}}^{q ; \mathbb{G}} \mathrm{v}(\mathfrak{t})\right)\right) & =\mathrm{v}_{3}+\mathcal{I}_{a^{+}}^{k ; \mathbb{G} c} \mathcal{D}_{a^{+}}^{k ; \mathfrak{G}}\left({ }^{c} \mathcal{D}_{a^{+}}^{r ; \mathbb{G}}\left({ }^{c} \mathcal{D}_{a^{+}}^{p ; \mathbb{G}}\left({ }^{c} \mathcal{D}_{a^{+}}^{q ; \mathbb{G}} \mathrm{v}(\mathfrak{t})\right)\right)\right) \\
& =\mathrm{v}_{3}+\mathcal{I}_{a^{+}}^{k ; \mathbb{G}} g(\mathfrak{t}) .
\end{aligned}
$$

Similarly, by the 3 rd boundary condition, applying the $r$-th $\mathbb{G}$-integral operator $\mathcal{I}_{a^{+}}^{r, \mathbb{G}}$, we get

$$
{ }^{c} \mathcal{D}_{a^{+}}^{p ; \mathbb{G}}\left({ }^{c} \mathcal{D}_{a^{+}}^{q ; \mathbb{G}} \mathrm{v}(\mathfrak{t})\right)=\mathrm{v}_{2}+\frac{\mathrm{v}_{3}(\mathbb{G}(\mathfrak{t})-\mathbb{G}(a))^{r}}{\Gamma(r+1)}+\mathcal{I}_{a^{+}}^{k+r ; \mathbb{G}} g(\mathfrak{t}) .
$$

By the 2nd boundary condition, applying the $p$ th $\mathbb{G}$-integral operator $\mathcal{I}_{a^{+}}^{p ; \mathbb{G}}$, we get

$$
{ }^{c} \mathcal{D}_{a^{+}}^{q ; \mathbb{G}} \mathrm{v}(\mathfrak{t})=\mathrm{v}_{1}+\frac{\mathrm{v}_{2}(\mathbb{G}(\mathfrak{t})-\mathbb{G}(a))^{p}}{\Gamma(p+1)}+\frac{\mathrm{v}_{3}(\mathbb{G}(\mathfrak{t})-\mathbb{G}(a))^{p+r}}{\Gamma(p+r+1)}+\mathcal{I}_{a^{+}}^{k+r+p ; \mathbb{G}} g(\mathfrak{t}),
$$


and finally, applying the $q$ th $\mathbb{G}$-integral operator $\mathcal{I}_{a^{+}}^{q ; \mathbb{G}}$ to both sides of (10), by the 1 st boundary condition, we get

$$
\begin{aligned}
\mathrm{v}(\mathfrak{t})= & \mathrm{v}_{0}+\frac{\mathrm{v}_{1}(\mathbb{G}(\mathfrak{t})-\mathbb{G}(a))^{q}}{\Gamma(q+1)}+\frac{\mathrm{v}_{2}(\mathbb{G}(\mathfrak{t})-\mathbb{G}(a))^{q+p}}{\Gamma(q+p+1)} \\
& +\frac{\mathrm{v}_{3}(\mathbb{G}(\mathfrak{t})-\mathbb{G}(a))^{q+p+r}}{\Gamma(q+p+r+1)}+\mathcal{I}_{a^{+}}^{k+r+p+q ; \mathbb{G}} g(\mathfrak{t}) .
\end{aligned}
$$

We see that $v(\mathfrak{t})$ fulfills (9), and the proof is complete.

At present, we aim to verify the existence of a unique solution of the fractional $\mathbb{G}$-snap system (4) by relying on Theorem 2.4. Note that $C([a, b], \mathbb{R})$ is a Banach space with norm

$$
\begin{aligned}
\|\mathrm{v}\|= & \sup _{\mathfrak{t} \in[a, b]}|\mathrm{v}(\mathfrak{t})|+\sup _{\mathfrak{t} \in[a, b]}\left|{ }^{c} \mathcal{D}_{a^{+}}^{q ; \mathbb{G}} \mathrm{v}(\mathfrak{t})\right|+\sup _{\mathfrak{t} \in[a, b]}\left|{ }^{c} \mathcal{D}_{a^{+}}^{p ; \mathbb{G}}\left({ }^{c} \mathcal{D}_{a^{+}}^{q ; \mathbb{G}} \mathrm{v}(\mathfrak{t})\right)\right| \\
& +\sup _{\mathfrak{t} \in[a, b]}\left|{ }^{c} \mathcal{D}_{a^{+}}^{r ; \mathbb{G}}\left({ }^{c} \mathcal{D}_{a^{+}}^{p ; \mathbb{G}}\left({ }^{c} \mathcal{D}_{a^{+}}^{q \mathbb{G}} \mathbf{v}(\mathfrak{t})\right)\right)\right|, \quad \forall \mathrm{v} \in C([a, b], \mathbb{R}) .
\end{aligned}
$$

Theorem 3.2 Let $h \in C\left([a, b] \times \mathbb{R}^{4}, \mathbb{R}\right)$, and let

(C1) $\exists L>0$ such that $\forall \mathfrak{t} \in[a, b]$ and $\mathrm{v}_{j}, \mathrm{v}_{j}^{*} \in C([a, b], \mathbb{R}), j=1,2,3,4$,

$$
\begin{aligned}
& \left|h\left(\mathfrak{t}, \mathrm{v}_{1}(\mathfrak{t}), \mathrm{v}_{2}(\mathfrak{t}), \mathrm{v}_{3}(\mathfrak{t}), \mathrm{v}_{4}(\mathfrak{t})\right)-h\left(\mathfrak{t}, \mathrm{v}_{1}^{*}(\mathfrak{t}), \mathrm{v}_{2}^{*}(\mathfrak{t}), \mathrm{v}_{3}^{*}(\mathfrak{t}), \mathrm{v}_{4}^{*}(\mathfrak{t})\right)\right| \\
& \quad \leq L \sum_{j=1}^{4}\left|\mathrm{v}_{j}(\mathfrak{t})-\mathrm{v}_{j}^{*}(\mathfrak{t})\right| .
\end{aligned}
$$

Then the fractional $\mathbb{G}$-snap system (4) admits a unique solution on $[a, b]$ if $L \mathcal{O}<1$, where

$$
\begin{aligned}
\mathcal{O}:= & \frac{(\mathbb{G}(b)-\mathbb{G}(a))^{q+p+r+k}}{\Gamma(q+p+r+k+1)}+\frac{(\mathbb{G}(b)-\mathbb{G}(a))^{p+r+k}}{\Gamma(p+r+k+1)} \\
& +\frac{(\mathbb{G}(b)-\mathbb{G}(a))^{r+k}}{\Gamma(r+k+1)}+\frac{(\mathbb{G}(b)-\mathbb{G}(a))^{k}}{\Gamma(k+1)} .
\end{aligned}
$$

Proof To prove the desired result, we first let

$$
\Omega_{\ell}=\{\mathrm{v} \in C([a, b], \mathbb{R}):\|\mathrm{v}\| \leq \ell\}
$$

for some constant $\ell>0$ satisfying

$$
\ell \geq \frac{\Lambda+h_{0}^{*} \mathcal{O}}{1-L \mathcal{O}}
$$

where $h_{0}^{*}=\sup _{\mathfrak{t} \in[a, b]}|h(t, 0,0,0,0)|$, and

$$
\begin{aligned}
\Lambda:= & \left|\mathrm{v}_{0}\right|+\left|\mathrm{v}_{1}\right|\left(1+\frac{(\mathbb{G}(b)-\mathbb{G}(a))^{q}}{\Gamma(q+1)}\right) \\
& +\left|\mathrm{v}_{2}\right|\left(1+\frac{(\mathbb{G}(b)-\mathbb{G}(a))^{p}}{\Gamma(p+1)}+\frac{(\mathbb{G}(b)-\mathbb{G}(a))^{q+p}}{\Gamma(q+p+1)}\right)
\end{aligned}
$$




$$
\begin{aligned}
& +\left|\mathrm{v}_{3}\right|\left(1+\frac{(\mathbb{G}(b)-\mathbb{G}(a))^{r}}{\Gamma(r+1)}+\frac{(\mathbb{G}(b)-\mathbb{G}(a))^{r+p}}{\Gamma(r+p+1)}\right. \\
& \left.+\frac{(\mathbb{G}(b)-\mathbb{G}(a))^{q+p+r}}{\Gamma(q+p+r+1)}\right) .
\end{aligned}
$$

To apply the Banach principle, we verify that $\Psi: C([a, b], \mathbb{R}) \rightarrow C([a, b], \mathbb{R})$ given as

$$
\begin{aligned}
(\Psi \mathrm{v})(\mathfrak{t})= & \mathcal{I}_{a^{+}}^{q+p+r+k ; \mathbb{G}} \hat{h}_{\mathrm{v}}(\mathfrak{t})+\mathrm{v}_{0}+\mathrm{v}_{1} \frac{(\mathbb{G}(\mathfrak{t})-\mathbb{G}(a))^{q}}{\Gamma(q+1)} \\
& +\mathrm{v}_{2} \frac{(\mathbb{G}(\mathfrak{t})-\mathbb{G}(a))^{p+q}}{\Gamma(p+q+1)}+\mathrm{v}_{3} \frac{(\mathbb{G}(\mathfrak{t})-\mathbb{G}(a))^{r+p+q}}{\Gamma(r+p+q+1)}
\end{aligned}
$$

where

$$
\hat{h}_{\mathrm{v}}(\mathfrak{t})=h\left(\mathfrak{t}, \mathrm{v}(\mathfrak{t}),{ }^{c} \mathcal{D}_{a^{+}}^{q ; \mathbb{G}} \mathrm{v}(\mathfrak{t}),{ }^{c} \mathcal{D}_{a^{+}}^{p ; \mathbb{G}}\left({ }^{c} \mathcal{D}_{a^{+}}^{q ; \mathbb{G}} \mathrm{v}(\mathfrak{t})\right),{ }^{c} \mathcal{D}_{a^{+}}^{r ; \mathbb{G}}\left({ }^{c} \mathcal{D}_{a^{+}}^{p ; \mathbb{G}}\left({ }^{c} \mathcal{D}_{a^{+}}^{q ; \mathbb{G}} \mathrm{v}(\mathfrak{t})\right)\right)\right)
$$

admits a unique fixed point, which is the same solution of the fractional $\mathbb{G}$-snap BVP (4). First, we show $\Psi \Omega_{\ell} \subset \Omega_{\ell}$, that is, $\Psi$ maps $\Omega_{\ell}$ into itself. For each $\mathrm{v} \in \Omega_{r}$, we have

$$
\begin{aligned}
|(\Psi \mathrm{v})(\mathfrak{t})| \leq & \left|\mathrm{v}_{0}\right|+\left|\mathrm{v}_{1}\right| \frac{(\mathbb{G}(\mathfrak{t})-\mathbb{G}(a))^{q}}{\Gamma(q+1)}+\left|\mathrm{v}_{2}\right| \frac{(\mathbb{G}(\mathfrak{t})-\mathbb{G}(a))^{p+q}}{\Gamma(p+q+1)} \\
& +\left|\mathrm{v}_{3}\right| \frac{(\mathbb{G}(\mathfrak{t})-\mathbb{G}(a))^{r+p+q}}{\Gamma(r+p+q+1)}+\mathcal{I}_{a^{+}}^{q+p+r+k ; \mathbb{G}}\left|\hat{h}_{\mathrm{v}}(\mathfrak{t})\right| \\
\leq & \left|\mathrm{v}_{0}\right|+\left|\mathrm{v}_{1}\right| \frac{(\mathbb{G}(\mathfrak{t})-\mathbb{G}(a))^{q}}{\Gamma(q+1)}+\left|\mathrm{v}_{2}\right| \frac{(\mathbb{G}(\mathfrak{t})-\mathbb{G}(a))^{p+q}}{\Gamma(p+q+1)} \\
& +\left|\mathrm{v}_{3}\right| \frac{(\mathbb{G}(\mathfrak{t})-\mathbb{G}(a))^{r+p+q}}{\Gamma(r+p+q+1)} \\
& +\mathcal{I}_{a^{+}}^{q+p+r+k ; \mathbb{G}}\left(\left|\hat{h}_{\mathrm{v}}(\mathfrak{t})-h(\mathfrak{t}, 0,0,0,0)\right|+|h(\mathfrak{t}, 0,0,0,0)|\right) \\
\leq & \left|\mathrm{v}_{0}\right|+\left|\mathrm{v}_{1}\right| \frac{(\mathbb{G}(\mathfrak{t})-\mathbb{G}(a))^{q}}{\Gamma(q+1)}+\left|\mathrm{v}_{2}\right| \frac{(\mathbb{G}(\mathfrak{t})-\mathbb{G}(a))^{p+q}}{\Gamma(p+q+1)} \\
& +\left|\mathrm{v}_{3}\right| \frac{(\mathbb{G}(\mathfrak{t})-\mathbb{G}(a))^{r+p+q}}{\Gamma(r+p+q+1)} \\
& +\mathcal{I}_{a^{+}}^{q+p+r+k ; \mathbb{G}}\left(L \left(|\mathrm{v}(\mathfrak{t})|+\left|{ }^{c} \mathcal{D}_{a^{+}}^{q ; \mathbb{G}} \mathrm{v}(\mathfrak{t})\right|+\left|{ }^{c} \mathcal{D}_{a^{+}}^{p ; \mathbb{G}}\left({ }^{c} \mathcal{D}_{a^{+}}^{q ; \mathbb{G}} \mathrm{v}(\mathfrak{t})\right)\right|\right.\right. \\
& \left.\left.\left.+\mid \mathcal{D}^{r ; \mathbb{G}}{ }_{a^{+}}{ }^{c} \mathcal{D}_{a^{+}}^{p ; \mathbb{G}}\left({ }^{c} \mathcal{D}_{a^{+}}^{q ;} \mathrm{G}(\mathfrak{t})\right)\right) \mid\right)+h_{0}^{*}\right) \\
\leq & \left|\mathrm{v}_{0}\right|+\left|\mathrm{v}_{1}\right| \frac{(\mathbb{G}(b)-\mathbb{G}(a))^{q}}{\Gamma(q+1)}+\left|\mathrm{v}_{2}\right| \frac{(\mathbb{G}(b)-\mathbb{G}(a))^{p+q}}{\Gamma(p+q+1)} \\
& +\left|\mathrm{v}_{3}\right| \frac{(\mathbb{G}(b)-\mathbb{G}(a))^{r+p+q}}{\Gamma(r+p+q+1)} \\
& +\left(L\|\mid \mathrm{v}\|+h_{0}^{*}\right) \frac{(\mathbb{G}(b)-\mathbb{G}(a))^{q+p+r+k}}{\Gamma(q+p+r+k+1)} \\
\leq & \left|\mathrm{v}_{0}\right|+\left|\mathrm{v}_{1}\right| \frac{(\mathbb{G}(b)-\mathbb{G}(a))^{q}}{\Gamma(q+1)}+\left|\mathrm{v}_{2}\right| \frac{(\mathbb{G}(b)-\mathbb{G}(a))^{p+q}}{\Gamma(p+q+1)} \\
& +\left|\mathrm{v}_{3}\right| \frac{(\mathbb{G}(b)-\mathbb{G}(a))^{r+p+q}}{\Gamma(r+p+q+1)} \\
&
\end{aligned}
$$




$$
+\left(L \ell+h_{0}^{*}\right) \frac{(\mathbb{G}(b)-\mathbb{G}(a))^{q+p+r+k}}{\Gamma(q+p+r+k+1)} .
$$

Also,

$$
\begin{aligned}
& \left|{ }^{c} \mathcal{D}_{a^{+}}^{q ; \mathbb{G}}(\Psi \mathrm{V})(\mathfrak{t})\right| \\
& \leq\left|\mathrm{v}_{1}\right|+\left|\mathrm{v}_{2}\right| \frac{(\mathbb{G}(\mathfrak{t})-\mathbb{G}(a))^{p}}{\Gamma(p+1)} \\
& +\left|\mathrm{v}_{3}\right| \frac{(\mathbb{G}(\mathfrak{t})-\mathbb{G}(a))^{r+p}}{\Gamma(r+p+1)}+\mathcal{I}_{a^{+}}^{p+r+k ; \mathbb{G}}\left|\hat{h}_{\mathrm{v}}(\mathfrak{t})\right| \\
& \leq\left|\mathrm{v}_{1}\right|+\left|\mathrm{v}_{2}\right| \frac{(\mathbb{G}(\mathfrak{t})-\mathbb{G}(a))^{p}}{\Gamma(p+1)}+\left|\mathrm{v}_{3}\right| \frac{(\mathbb{G}(\mathfrak{t})-\mathbb{G}(a))^{r+p}}{\Gamma(r+p+1)} \\
& +\mathcal{I}_{a^{+}}^{p+r+k ; \mathbb{G}}\left(\left|\hat{h}_{\mathrm{v}}(\mathfrak{t})-h(t, 0,0,0,0)\right|+|h(t, 0,0,0,0)|\right) \\
& \leq\left|\mathrm{v}_{1}\right|+\left|\mathrm{v}_{2}\right| \frac{(\mathbb{G}(\mathfrak{t})-\mathbb{G}(a))^{p}}{\Gamma(p+1)}+\left|\mathrm{v}_{3}\right| \frac{(\mathbb{G}(\mathfrak{t})-\mathbb{G}(a))^{r+p}}{\Gamma(r+p+1)} \\
& +\mathcal{I}_{a^{+}}^{p+r+k ; \mathbb{G}}\left(L \left(|\mathrm{v}(\mathfrak{t})|+\left|{ }^{c} \mathcal{D}_{a^{+}}^{q ; \mathbb{G}} \mathrm{v}(\mathfrak{t})\right|+\left|{ }^{c} \mathcal{D}_{a^{+}}^{p ; \mathbb{G}}\left({ }^{c} \mathcal{D}_{a^{+}}^{q ; \mathbb{G}} \mathrm{v}(\mathfrak{t})\right)\right|\right.\right. \\
& \left.\left.+\left|{ }^{c} \mathcal{D}_{a^{+}}^{r: \mathbb{G}}\left({ }^{c} \mathcal{D}_{a^{+}}^{p ; \mathbb{G}}\left({ }^{c} \mathcal{D}_{a^{+}}^{q ; \mathfrak{G}} \mathrm{v}(\mathfrak{t})\right)\right)\right|\right)+h_{0}^{*}\right) \\
& q \leq\left|\mathrm{v}_{1}\right|+\left|\mathrm{v}_{2}\right| \frac{(\mathbb{G}(b)-\mathbb{G}(a))^{p}}{\Gamma(p+1)}+\left|\mathrm{v}_{3}\right| \frac{(\mathbb{G}(\mathfrak{t})-\mathbb{G}(a))^{r+p}}{\Gamma(r+p+1)} \\
& +\left(L \ell+h_{0}^{*}\right) \frac{(\mathbb{G}(b)-\mathbb{G}(a))^{p+r+k}}{\Gamma(p+r+k+1)}, \\
& \left|{ }^{c} \mathcal{D}_{a^{+}}^{p ; \mathbb{G}}\left({ }^{c} \mathcal{D}_{a^{+}}^{q ; \mathbb{G}}(\Psi \mathrm{v})\right)(\mathfrak{t})\right| \\
& \leq\left|\mathrm{v}_{2}\right|+\left|\mathrm{v}_{3}\right| \frac{(\mathbb{G}(\mathfrak{t})-\mathbb{G}(a))^{r}}{\Gamma(r+1)}+\mathcal{I}_{a^{+}}^{r+k ; \mathbb{G}}\left|\hat{h}_{\mathrm{v}}(\mathfrak{t})\right| \\
& \leq\left|\mathrm{v}_{2}\right|+\left|\mathrm{v}_{3}\right| \frac{(\mathbb{G}(\mathfrak{t})-\mathbb{G}(a))^{r}}{\Gamma(r+1)} \\
& +\mathcal{I}_{a^{+}}^{r+k ; \mathbb{G}}\left(L \left(|\mathrm{v}(\mathfrak{t})|+\left|{ }^{c} \mathcal{D}_{a^{+}}^{q ; \mathbb{G}} \mathrm{v}(\mathfrak{t})\right|+\left|{ }^{c} \mathcal{D}_{a^{+}}^{p ; \mathbb{G}}\left({ }^{c} \mathcal{D}_{a^{+}}^{q ; \mathbb{G}} \mathrm{v}(\mathfrak{t})\right)\right|\right.\right. \\
& \left.\left.+\left|{ }^{c} \mathcal{D}_{a^{+}}^{r: \mathbb{G}}\left({ }^{c} \mathcal{D}_{a^{+}}^{p ; \mathbb{G}}\left({ }^{c} \mathcal{D}_{a^{+}}^{q ; \mathbb{G}} \mathrm{v}(\mathfrak{t})\right)\right)\right|\right)+h_{0}^{*}\right) \\
& \leq\left|\mathrm{v}_{2}\right|+\left|\mathrm{v}_{3}\right| \frac{(\mathbb{G}(\mathfrak{t})-\mathbb{G}(a))^{r}}{\Gamma(r+1)}+\left(L \ell+h_{0}^{*}\right) \frac{(\mathbb{G}(b)-\mathbb{G}(a))^{r+k}}{\Gamma(r+k+1)},
\end{aligned}
$$

and

$$
\begin{aligned}
\mid{ }^{c} & \mathcal{D}_{a^{+}}^{r ; \mathbb{G}}\left({ }^{c} \mathcal{D}_{a^{+}}^{p ; \mathbb{G}}\left({ }^{c} \mathcal{D}_{a^{+}}^{q ; \mathbb{G}}(\Psi \mathrm{v})\right)\right)(\mathfrak{t}) \mid \\
\leq & \left|\mathrm{v}_{3}\right|+\mathcal{I}_{a^{+}}^{k ; \mathbb{G}}\left|\hat{h}_{\mathrm{v}}(\mathfrak{t})\right| \\
\leq & \left|\mathrm{v}_{3}\right|+\mathcal{I}_{a^{+}}^{k ; \mathbb{G}}\left(L \left(|\mathrm{v}(\mathfrak{t})|+\left|{ }^{c} \mathcal{D}_{a^{+}}^{q ; \mathbb{G}} \mathrm{v}(\mathfrak{t})\right|+\left|{ }^{c} \mathcal{D}_{a^{+}}^{p ; \mathbb{G}}\left({ }^{c} \mathcal{D}_{a^{+}}^{q ; \mathbb{G}} \mathrm{v}(\mathfrak{t})\right)\right|\right.\right. \\
& \left.\left.+\left|{ }^{c} \mathcal{D}_{a^{+}}^{r ; \mathbb{G}}\left({ }^{c} \mathcal{D}_{a^{+}}^{p ; \mathbb{G}}\left({ }^{c} \mathcal{D}_{a^{+}}^{q ; \mathfrak{G}} \mathrm{v}(\mathfrak{t})\right)\right)\right|\right)+h_{0}^{*}\right) \\
\leq & \left|\mathrm{v}_{3}\right|+\left(L \ell+h_{0}^{*}\right) \frac{(\mathbb{G}(b)-\mathbb{G}(a))^{k}}{\Gamma(k+1)} .
\end{aligned}
$$


From (16), (17), (18), (19), and (13) we get

$$
\begin{aligned}
\|\Psi \mathrm{v}\|= & \sup _{\mathfrak{t} \in[a, b]}\left(|(\Psi \mathrm{x})(\mathfrak{t})|+\left|{ }^{c} \mathcal{D}_{a^{+}}^{q ; \mathbb{G}}(\Psi \mathrm{v})(\mathfrak{t})\right|+\left|{ }^{c} \mathcal{D}_{a^{+}}^{p ; \mathbb{G}}\left({ }^{c} \mathcal{D}_{a^{+}}^{q ; \mathbb{G}}(\Psi \mathrm{v})\right)(\mathfrak{t})\right|\right. \\
& \left.+\left|{ }^{c} \mathcal{D}_{a^{+}}^{r ; \mathbb{G}}\left({ }^{c} \mathcal{D}_{a^{+}}^{p ; \mathbb{G}}\left({ }^{c} \mathcal{D}_{a^{+}}^{q ; \mathbb{G}}(\Psi \mathrm{v})\right)\right)(\mathfrak{t})\right|\right) \\
\leq & {\left[\left|\mathrm{v}_{0}\right|+\left|\mathrm{v}_{1}\right|\left(1+\frac{(\mathbb{G}(b)-\mathbb{G}(a))^{q}}{\Gamma(q+1)}\right)\right.} \\
& +\left|\mathrm{v}_{2}\right|\left(1+\frac{(\mathbb{G}(b)-\mathbb{G}(a))^{p}}{\Gamma(p+1)}+\frac{(\mathbb{G}(b)-\mathbb{G}(a))^{p+q}}{\Gamma(p+q+1)}\right) \\
& +\left|\mathrm{v}_{3}\right|\left(1+\frac{(\mathbb{G}(b)-\mathbb{G}(a))^{r}}{\Gamma(r+1)}+\frac{(\mathbb{G}(b)-\mathbb{G}(a))^{r+p}}{\Gamma(r+p+1)}\right. \\
& \left.\left.+\frac{(\mathbb{G}(b)-\mathbb{G}(a))^{q+p+r}}{\Gamma(q+p+r+1)}\right)\right]+\left(L \ell+h_{0}^{*}\right)\left[\frac{(\mathbb{G}(b)-\mathbb{G}(a))^{q+p+r+k}}{\Gamma(q+p+r+k+1)}\right. \\
& \left.+\frac{(\mathbb{G}(b)-\mathbb{G}(a))^{p+r+k}}{\Gamma(p+r+k+1)}+\frac{\left.(\mathbb{G}(b)-\mathbb{G}(a))^{r+k}+\frac{(\mathbb{G}(b)-\mathbb{G}(a))^{k}}{\Gamma(r+k+1)}\right]}{\Gamma(k+1)}\right] \\
= & \Lambda+\left(L \ell+h_{0}^{*}\right) \mathcal{O}<\ell,
\end{aligned}
$$

which implies that $\|\Psi \mathrm{v}\| \leq \ell$ for $\mathrm{v} \in \Omega_{\ell}$, and so $\Psi \Omega_{\ell} \subset \Omega_{\ell}$. Next, we investigate the contractivity property of the operator $\Psi$. For $\mathrm{v}, \mathrm{w} \in C([a, b], \mathbb{R})$, we estimate

$$
\begin{aligned}
& \left|\left(\Psi_{\mathrm{v}}\right)(\mathfrak{t})-\left(\Psi_{\mathrm{W}}\right)(\mathfrak{t})\right| \\
& \leq \mathcal{I}_{a^{+}}^{q+p+r+k ; \mathbb{G}}\left|\hat{h}_{\mathrm{v}}(\mathfrak{t})-\hat{h}_{\mathrm{W}}(\mathfrak{t})\right| \\
& \leq \mathcal{I}_{a^{+}}^{q+p+r+k ; \mathbb{G}} L\left(|\mathrm{v}(\mathfrak{t})-\mathrm{w}(\mathfrak{t})|+\left|{ }^{c} \mathcal{D}_{a^{+}}^{q ; \mathbb{G}} \mathrm{v}(\mathfrak{t})-{ }^{c} \mathcal{D}_{a^{+}}^{q ; \mathbb{G}} \mathrm{W}(\mathfrak{t})\right|\right. \\
& +\left|{ }^{c} \mathcal{D}_{a^{+}}^{p ; \mathbb{G}}\left({ }^{c} \mathcal{D}_{a^{+}}^{q ; \mathbb{G}} \mathrm{v}(\mathfrak{t})\right)-{ }^{c} \mathcal{D}_{a^{+}}^{p ; \mathbb{G}}\left({ }^{c} \mathcal{D}_{a^{+}}^{q ; \mathbb{W}} \mathrm{w}(\mathfrak{t})\right)\right| \\
& \left.+\left|{ }^{c} \mathcal{D}_{a^{+}}^{r ; \mathbb{G}}\left({ }^{c} \mathcal{D}_{a^{+}}^{p ; \mathbb{G}}\left({ }^{c} \mathcal{D}_{a^{+}}^{q ; \mathbb{G}} \mathrm{v}(\mathfrak{t})\right)\right)-{ }^{c} \mathcal{D}_{a^{+}}^{r ; \mathbb{G}}\left({ }^{c} \mathcal{D}_{a^{+}}^{p ; \mathbb{G}}\left({ }^{c} \mathcal{D}_{a^{+}}^{q ; \mathbb{G}} \mathrm{W}(\mathfrak{t})\right)\right)\right|\right) \\
& \leq L \frac{(\mathbb{G}(b)-\mathbb{G}(a))^{q+p+r+k}}{\Gamma(q+p+r+k+1)}\|\mathrm{v}-\mathrm{w}\|, \\
& \left|{ }^{c} \mathcal{D}_{a^{+}}^{q ; \mathbb{G}}\left(\Psi_{\mathrm{V}}\right)(\mathfrak{t})-{ }^{c} \mathcal{D}_{a^{+}}^{q ; \mathbb{G}}\left(\Psi_{\mathrm{W}}\right)(\mathfrak{t})\right| \\
& \leq \mathcal{I}_{a^{+}}^{p+r+k ; \mathbb{G}}\left|\hat{h}_{\mathrm{v}}-\hat{h}_{\mathrm{w}}\right| \\
& \leq \mathcal{I}_{a^{+}}^{p+r+k ; \mathbb{G}} L\left(|\mathrm{v}(\mathfrak{t})-\mathrm{w}(\mathfrak{t})|+\left|{ }^{c} \mathcal{D}_{a^{+}}^{q ; \mathbb{G}} \mathrm{x}(\mathfrak{t})-{ }^{c} \mathcal{D}_{a^{+}}^{q ; \mathbb{G}} \mathrm{W}(\mathfrak{t})\right|\right. \\
& +\left|{ }^{c} \mathcal{D}_{a^{+}}^{p ; \mathbb{G}}\left({ }^{c} \mathcal{D}_{a^{+}}^{q ; \mathbb{G}} \mathrm{v}(\mathfrak{t})\right)-{ }^{c} \mathcal{D}_{a^{+}}^{p ; \mathbb{G}}\left({ }^{c} \mathcal{D}_{a^{+}}^{q ; \mathbb{G}} \mathrm{w}(\mathfrak{t})\right)\right| \\
& \left.+\left|{ }^{c} \mathcal{D}_{a^{+}}^{r ; \mathbb{G}}\left({ }^{c} \mathcal{D}_{a^{+}}^{p ; \mathbb{G}}\left({ }^{c} \mathcal{D}_{a^{+}}^{q ; \mathbb{G}} \mathrm{v}(\mathfrak{t})\right)\right)-{ }^{c} \mathcal{D}_{a^{+}}^{r ; \mathbb{G}}\left({ }^{c} \mathcal{D}_{a^{+}}^{p ; \mathbb{G}}\left({ }^{c} \mathcal{D}_{a^{+}}^{q ; \mathbb{W}} \mathrm{W}(\mathfrak{t})\right)\right)\right|\right) \\
& \leq L \frac{(\mathbb{G}(b)-\mathbb{G}(a))^{p+r+k}}{\Gamma(p+r+k+1)}\|\mathrm{v}-\mathrm{w}\|, \\
& \left|{ }^{c} \mathcal{D}_{a^{+}}^{p ; \mathbb{G}}\left({ }^{c} \mathcal{D}_{a^{+}}^{q ; \mathbb{G}}(\Psi \mathrm{v})\right)(\mathfrak{t})-{ }^{c} \mathcal{D}_{a^{+}}^{p ; \mathbb{G}}\left({ }^{c} \mathcal{D}_{a^{+}}^{q ; \mathbb{G}}(\Psi \mathrm{W})\right)(\mathfrak{t})\right| \\
& \leq \mathcal{I}_{a^{+}}^{r+k ; \mathbb{G}}\left|\hat{h}_{\mathrm{v}}-\hat{h}_{\mathrm{w}}\right| \\
& \leq \mathcal{I}_{a^{+}}^{r+k ; \mathbb{G}} L\left(|\mathrm{v}(\mathfrak{t})-\mathrm{w}(\mathfrak{t})|+\left|{ }^{c} \mathcal{D}_{a^{+}}^{q ; \mathfrak{G}} \mathrm{v}(\mathfrak{t})-{ }^{c} \mathcal{D}_{a^{+}}^{q ; \mathfrak{G}} \mathrm{w}(\mathfrak{t})\right|\right. \\
& +\left|{ }^{c} \mathcal{D}_{a^{+}}^{p ; \mathbb{G}}\left({ }^{c} \mathcal{D}_{a^{+}}^{q ; \mathbb{G}} \mathrm{v}(\mathfrak{t})\right)-{ }^{c} \mathcal{D}_{a^{+}}^{p ; \mathbb{G}}\left({ }^{c} \mathcal{D}_{a^{+}}^{q ; \mathbb{W}} \mathrm{W}(\mathfrak{t})\right)\right|
\end{aligned}
$$




$$
\begin{aligned}
& \left.+\left|{ }^{c} \mathcal{D}_{a^{+}}^{r, \mathbb{G}}\left({ }^{c} \mathcal{D}_{a^{+}}^{p ; \mathbb{G}}\left({ }^{c} \mathcal{D}_{a^{+}}^{q ; \mathbb{G}} \mathrm{v}(\mathfrak{t})\right)\right)-{ }^{c} \mathcal{D}_{a^{+}}^{r ; \mathbb{G}}\left({ }^{c} \mathcal{D}_{a^{+}}^{p ; \mathbb{G}}\left({ }^{c} \mathcal{D}_{a^{+}}^{q ; \mathbb{G}} \mathrm{w}(\mathfrak{t})\right)\right)\right|\right) \\
\leq & L \frac{(\mathbb{G}(b)-\mathbb{G}(a))^{r+k}}{\Gamma(r+k+1)}\|\mathrm{v}-\mathrm{w}\|,
\end{aligned}
$$

and

$$
\begin{aligned}
&\left|{ }^{c} \mathcal{D}_{a^{+}}^{r ; \mathbb{G}}\left({ }^{c} \mathcal{D}_{a^{+}}^{p ; \mathbb{G}}\left({ }^{c} \mathcal{D}_{a^{+}}^{q ; \mathbb{G}}(\Psi \mathrm{v})\right)\right)(\mathfrak{t})-{ }^{c} \mathcal{D}_{a^{+}}^{r ; \mathbb{G}}\left({ }^{c} \mathcal{D}_{a^{+}}^{p ; \mathbb{G}}\left({ }^{c} \mathcal{D}_{a^{+}}^{q ; \mathbb{G}}(\Psi \mathrm{w})\right)\right)(\mathfrak{t})\right| \\
& \leq \leq \mathcal{I}_{a^{+}}^{k ; \mathbb{G}}\left|\hat{h}_{\mathrm{v}}-\hat{h}_{\mathrm{w}}\right| \\
& \leq \mathcal{I}_{a^{+}}^{k ; \mathbb{G}} L\left(|\mathrm{v}(\mathfrak{t})-\mathrm{w}(\mathfrak{t})|+\left|{ }^{c} \mathcal{D}_{a^{+}}^{q ; \mathbb{G}} \mathrm{v}(\mathfrak{t})-{ }^{c} \mathcal{D}_{a^{+}}^{q ; \mathbb{G}} \mathrm{w}(\mathfrak{t})\right|\right. \\
&+\left|{ }^{c} \mathcal{D}_{a^{+}}^{p ; \mathbb{G}}\left({ }^{c} \mathcal{D}_{a^{+}}^{q ; \mathbb{G}} \mathrm{v}(\mathfrak{t})\right)-{ }^{c} \mathcal{D}_{a^{+}}^{p ; \mathbb{G}}\left({ }^{c} \mathcal{D}_{a^{+}}^{q ; \mathbb{G}} \mathrm{w}(\mathfrak{t})\right)\right| \\
&\left.+\left|{ }^{c} \mathcal{D}_{a^{+}}^{r ; \mathbb{G}}\left({ }^{c} \mathcal{D}_{a^{+}}^{p ; \mathbb{G}}\left({ }^{c} \mathcal{D}_{a^{+}}^{q ; \mathbb{G}} \mathrm{v}(\mathfrak{t})\right)\right)-{ }^{c} \mathcal{D}_{a^{+}}^{r ; \mathbb{G}}\left({ }^{c} \mathcal{D}_{a^{+}}^{p ; \mathbb{G}}\left({ }^{c} \mathcal{D}_{a^{+}}^{q ; \mathbb{G}} \mathrm{w}(\mathfrak{t})\right)\right)\right|\right) \\
& \leq L \frac{(\mathbb{G}(b)-\mathbb{G}(a))^{k}}{\Gamma(k+1)}\|\mathrm{v}-\mathrm{w}\| .
\end{aligned}
$$

From (20), (21), (22), and (23) we obtain

$$
\begin{aligned}
\|\Psi \mathrm{v}-\Psi \mathrm{w}\| \leq & L\left[\frac{(\mathbb{G}(b)-\mathbb{G}(a))^{q+p+r+k}}{\Gamma(q+p+r+k+1)}+\frac{(\mathbb{G}(b)-\mathbb{G}(a))^{p+r+k}}{\Gamma(p+r+k+1)}\right. \\
& \left.+\frac{(\mathbb{G}(b)-\mathbb{G}(a))^{r+k}}{\Gamma(r+k+1)}+\frac{(\mathbb{G}(b)-\mathbb{G}(a))^{k}}{\Gamma(k+1)}\right]\|\mathrm{v}-\mathrm{w}\| \\
= & L \mathcal{O}\|\mathrm{v}-\mathrm{w}\| .
\end{aligned}
$$

Thus $\|\Psi \mathrm{v}-\Psi \mathrm{w}\| \leq L \mathcal{O}\|\mathrm{v}-\mathrm{w}\|$. Since $L \mathcal{O}<1, \Psi$ is a contraction on $C([a, b], \mathbb{R})$. This, together with Theorem 2.4, guarantees the existence of a unique fixed point for $\Psi$ and accordingly the existence of a unique solution for the fractional $\mathbb{G}$-snap BVP (4). The proof is complete.

The next existence property for possible solutions of the fractional $\mathbb{G}$-snap BVP (4) is checked based on the hypotheses of Theorem 2.5.

Theorem 3.3 Let $h \in C\left([a, b] \times \mathbb{R}^{4}, \mathbb{R}\right)$ and assume that:

(C2) there exist $\varrho \in L^{1}\left([a, b], \mathbb{R}^{+}\right)$and an increasing function $f \in C([0, \infty),(0, \infty))$ such that for all $\mathfrak{t} \in[a, b]$ and $\mathrm{v}_{j} \in C([a, b], \mathbb{R}), j=1,2,3,4$,

$$
\left|h\left(\mathfrak{t}, \mathrm{v}_{1}(\mathfrak{t}), \mathrm{v}_{2}(\mathfrak{t}), \mathrm{v}_{3}(\mathfrak{t}), \mathrm{v}_{4}(\mathfrak{t})\right)\right| \leq \varrho(\mathfrak{t}) f\left(\sum_{j=1}^{4}\left|\mathrm{v}_{j}(\mathfrak{t})\right|\right)
$$

(C3) there exists $B>0$ such that

$$
\frac{B}{\Lambda+\mathcal{O} \varrho_{0}^{*} f(B)}>1
$$

where $\varrho_{0}^{*}=\sup _{\mathfrak{t} \in[a, b]}|\varrho(\mathfrak{t})|$, and $\mathcal{O}$ and $\Lambda$ are represented in (12) and (14).

Then the fractional $\mathbb{G}$-snap system (4) has at least one solution on $[a, b]$. 
Proof consider $\Psi: C([a, b], \mathbb{R}) \rightarrow C([a, b], \mathbb{R})$ defined by (15) and the ball $N_{\epsilon}=\{\mathrm{v} \in$ $C([a, b], \mathbb{R}):\|\mathrm{v}\| \leq \epsilon\}$ for some $\epsilon>0$. The continuity of $h$ yields that of the operator $\Psi$. Now by (C2) we have

$$
\begin{aligned}
& |(\Psi \mathrm{v})(\mathfrak{t})| \leq\left|\mathrm{v}_{0}\right|+\left|\mathrm{v}_{1}\right| \frac{(\mathbb{G}(\mathfrak{t})-\mathbb{G}(a))^{q}}{\Gamma(q+1)}+\left|\mathrm{v}_{2}\right| \frac{(\mathbb{G}(\mathfrak{t})-\mathbb{G}(a))^{p+q}}{\Gamma(p+q+1)} \\
& +\left|\mathrm{v}_{3}\right| \frac{(\mathbb{G}(\mathfrak{t})-\mathbb{G}(a))^{r+p+q}}{\Gamma(r+p+q+1)}+\mathcal{I}_{a^{+}}^{q+p+r+k ; \mathbb{G}}\left|\hat{h}_{\mathrm{v}}(\mathfrak{t})\right| \\
& \leq\left|\mathrm{v}_{0}\right|+\left|\mathrm{v}_{1}\right| \frac{(\mathbb{G}(\mathfrak{t})-\mathbb{G}(a))^{q}}{\Gamma(q+1)}+\left|\mathrm{v}_{2}\right| \frac{(\mathbb{G}(\mathfrak{t})-\mathbb{G}(a))^{p+q}}{\Gamma(p+q+1)} \\
& +\left|\mathrm{v}_{3}\right| \frac{(\mathbb{G}(\mathfrak{t})-\mathbb{G}(a))^{r+p+q}}{\Gamma(r+p+q+1)} \\
& +\mathcal{I}_{a^{+}}^{q+p+r+k ; \mathbb{G}} \varrho(\mathfrak{t}) f\left(|\mathrm{v}(\mathfrak{t})|+\left|{ }^{c} \mathcal{D}_{a^{+}}^{q ; \mathfrak{G}} \mathrm{v}(\mathfrak{t})\right|\right. \\
& \left.+\left|{ }^{c} \mathcal{D}_{a^{+}}^{p ; \mathbb{G}}\left({ }^{c} \mathcal{D}_{a^{+}}^{q ; \mathbb{G}} \mathrm{v}(\mathfrak{t})\right)\right|+\mid{ }^{c} \mathcal{D}_{a^{+}}^{r ; \mathbb{G}}\left({ }^{c} \mathcal{D}_{a^{+}}^{p ; \mathbb{G}}\left({ }^{c} \mathcal{D}_{a^{+}}^{q ; \mathbb{G}} \mathrm{v}(\mathfrak{t})\right)\right)\right) \\
& \leq\left|\mathrm{v}_{0}\right|+\left|\mathrm{v}_{1}\right| \frac{(\mathbb{G}(b)-\mathbb{G}(a))^{q}}{\Gamma(q+1)}+\left|\mathrm{v}_{2}\right| \frac{(\mathbb{G}(b)-\mathbb{G}(a))^{p+q}}{\Gamma(p+q+1)} \\
& +\frac{(\mathbb{G}(b)-\mathbb{G}(a))^{q+p+r}}{\Gamma(q+p+r+1)} \phi_{0}^{*} \varphi(\|\mathrm{v}\|) \\
& \leq\left|\mathrm{v}_{0}\right|+\left|\mathrm{v}_{1}\right| \frac{(\mathbb{G}(b)-\mathbb{G}(a))^{q}}{\Gamma(q+1)}+\left|\mathrm{v}_{2}\right| \frac{(\mathbb{G}(b)-\mathbb{G}(a))^{p+q}}{\Gamma(p+q+1)} \\
& +\left|\mathrm{v}_{3}\right| \frac{(\mathbb{G}(\mathfrak{t})-\mathbb{G}(a))^{r+p+q}}{\Gamma(r+p+q+1)}+\frac{(\mathbb{G}(b)-\mathbb{G}(a))^{q+p+r+k}}{\Gamma(q+p+r+k+1)} \varrho_{0}^{*} f(\epsilon)
\end{aligned}
$$

for $v \in N_{\epsilon}$. In a similar way, we get that

$$
\begin{aligned}
& \left|\mathcal{D}_{a^{+}}^{q ; \mathbb{G}}(\Psi \mathrm{v})(\mathfrak{t})\right| \\
& \leq\left|\mathrm{v}_{1}\right|+\left|\mathrm{v}_{2}\right| \frac{(\mathbb{G}(b)-\mathbb{G}(a))^{p}}{\Gamma(p+1)} \\
& \quad+\left|\mathrm{v}_{3}\right| \frac{(\mathbb{G}(\mathfrak{t})-\mathbb{G}(a))^{r+p}}{\Gamma(r+p+1)}+\frac{(\mathbb{G}(b)-\mathbb{G}(a))^{p+r+k}}{\Gamma(p+r+k+1)} \varrho_{0}^{*} f(\epsilon), \\
& \left|{ }^{c} \mathcal{D}_{a^{+}}^{p ; \mathbb{G}}\left({ }^{c} \mathcal{D}_{a^{+}}^{q ; \mathbb{G}}(\Psi \mathrm{v})\right)(\mathfrak{t})\right| \\
& \leq\left|\mathrm{v}_{2}\right|+\left|\mathrm{v}_{3}\right| \frac{(\mathbb{G}(\mathfrak{t})-\mathbb{G}(a))^{r}}{\Gamma(r+1)} \\
& \quad+\frac{(\mathbb{G}(b)-\mathbb{G}(a))^{r+k}}{\Gamma(r+k+1)} \varrho_{0}^{*} f(\epsilon),
\end{aligned}
$$

and

$$
\left|{ }^{c} \mathcal{D}_{a^{+}}^{r, \mathbb{G}}\left({ }^{c} \mathcal{D}_{a^{+}}^{p ; \mathbb{G}}\left({ }^{c} \mathcal{D}_{a^{+}}^{q ; \mathbb{G}}(\Psi \mathrm{v})\right)\right)(\mathfrak{t})\right| \leq\left|\mathrm{v}_{3}\right|+\frac{(\mathbb{G}(b)-\mathbb{G}(a))^{k}}{\Gamma(k+1)} \varrho_{0}^{*} f(\epsilon)
$$

As a consequence, by (25), (26), (27), and (28) we obtain

$$
\|\Psi \mathrm{v}\| \leq \Lambda+\mathcal{O} \varrho_{0}^{*} f(\epsilon)<\infty
$$


where $\mathcal{O}$ and $\Lambda$ are represented by (12) and (14). Hence $\Psi$ is uniformly bounded on $C([a, b], \mathbb{R})$. Now let us check the equicontinuity of $\Psi$. Choose arbitrary $\mathfrak{t}, \mathfrak{t}^{*} \in[a, b]$ with $t<t^{*}$ and $\mathrm{v} \in N_{\epsilon}$. We have

$$
\begin{aligned}
\left|(\Psi \mathrm{v})\left(\mathfrak{t}^{*}\right)-(\Psi \mathrm{v})(\mathfrak{t})\right| \leq & \left|\mathrm{v}_{1}\right| \frac{\left|\left(\mathbb{G}\left(\mathfrak{t}^{*}\right)-\mathbb{G}(a)\right)^{q}-(\mathbb{G}(\mathfrak{t})-\mathbb{G}(a))^{q}\right|}{\Gamma(q+1)} \\
& +\left|\mathrm{v}_{2}\right| \frac{\left|\left(\mathbb{G}\left(\mathfrak{t}^{*}\right)-\mathbb{G}(a)\right)^{p+q}-(\mathbb{G}(\mathfrak{t})-\mathbb{G}(a))^{p+q}\right|}{\Gamma(p+q+1)} \\
& +\left|\mathrm{v}_{3}\right| \frac{\left|\left(\mathbb{G}\left(\mathfrak{t}^{*}\right)-\mathbb{G}(a)\right)^{p+q+r}-(\mathbb{G}(\mathfrak{t})-\mathbb{G}(a))^{p+q+r}\right|}{\Gamma(p+q+r+1)} \\
& +\left|\mathcal{I}_{a^{+}}^{q+p+r+k ; \mathbb{G}} \hat{h}_{\mathrm{v}}\left(\mathfrak{t}^{*}\right)-\mathcal{I}_{a^{+}}^{q+p+r+k ; \mathbb{G}} \hat{h}_{\mathrm{v}}(\mathfrak{t})\right| .
\end{aligned}
$$

By letting

$$
\sup _{(\mathfrak{t}, \mathrm{v}, \mathrm{w}, \mathrm{x}, \mathrm{y}) \in[a, b] \times N_{\epsilon}^{4}}|h(\mathfrak{t}, \mathrm{v}, \mathrm{w}, \mathrm{x}, \mathrm{y})|=\tilde{H}<\infty,
$$

this becomes

$$
\begin{aligned}
\mid(\Psi \mathrm{V}) & \left(\mathfrak{t}^{*}\right)-(\Psi \mathrm{v})(\mathfrak{t}) \mid \\
\leq & \left|\mathrm{v}_{1}\right| \frac{\left|\left(\mathbb{G}\left(\mathfrak{t}^{*}\right)-\mathbb{G}(a)\right)^{q}-(\mathbb{G}(\mathfrak{t})-\mathbb{G}(a))^{q}\right|}{\Gamma(q+1)} \\
& +\left|\mathrm{v}_{2}\right| \frac{\left|\left(\mathbb{G}\left(\mathfrak{t}^{*}\right)-\mathbb{G}(a)\right)^{p+q}-(\mathbb{G}(\mathfrak{t})-\mathbb{G}(a))^{p+q}\right|}{\Gamma(p+q+1)} \\
& +\left|\mathrm{v}_{3}\right| \frac{\left|\left(\mathbb{G}\left(\mathfrak{t}^{*}\right)-\mathbb{G}(a)\right)^{p+q+r}-(\mathbb{G}(\mathfrak{t})-\mathbb{G}(a))^{p+q+r}\right|}{\Gamma(p+q+r+1)} \\
& +\frac{\tilde{H}}{\Gamma(q+p+r+k+1)}\left[\mid\left(\mathbb{G}\left(\mathfrak{t}^{*}\right)-\mathbb{G}(a)\right)^{q+p+r+k}\right. \\
& \left.-(\mathbb{G}(\mathfrak{t})-\mathbb{G}(a))^{q+p+r+k} \mid+2\left(\mathbb{G}\left(\mathfrak{t}^{*}\right)-\mathbb{G}(\mathfrak{t})\right)^{q+p+r+k}\right] .
\end{aligned}
$$

Obviously, the right-hand side of (30) does not depend on $v$ and approaches 0 as $t^{*}$ tends to $t$. In the same way,

$$
\begin{aligned}
\mid{ }^{c} \mathcal{D}_{a^{+}}^{q ; \mathbb{G}} & (\Psi \mathrm{v})\left(\mathfrak{t}^{*}\right)-{ }^{c} \mathcal{D}_{a^{+}}^{q ; \mathbb{G}}(\Psi \mathrm{v})(\mathfrak{t}) \mid \\
\leq & \left|\mathrm{v}_{2}\right| \frac{\left|\left(\mathbb{G}\left(\mathfrak{t}^{*}\right)-\mathbb{G}(a)\right)^{p}-(\mathbb{G}(\mathfrak{t})-\mathbb{G}(a))^{p}\right|}{\Gamma(p+1)} \\
& +\left|\mathrm{v}_{3}\right| \frac{\left|\left(\mathbb{G}\left(\mathfrak{t}^{*}\right)-\mathbb{G}(a)\right)^{p+r}-(\mathbb{G}(\mathfrak{t})-\mathbb{G}(a))^{p+r}\right|}{\Gamma(p+r+1)} \\
& +\left|\mathcal{I}_{a^{+}}^{p+r+k ; \mathbb{G}} h_{\mathrm{v}}\left(\mathfrak{t}^{*}\right)-\mathcal{I}_{a^{+}}^{p+r+k ; \mathbb{G}} h_{\mathrm{v}}(\mathfrak{t})\right| \\
\leq & \left|\mathrm{v}_{2}\right| \frac{\left|\left(\mathbb{G}\left(\mathfrak{t}^{*}\right)-\mathbb{G}(a)\right)^{p}-(\mathbb{G}(\mathfrak{t})-\mathbb{G}(a))^{p}\right|}{\Gamma(p+1)} \\
& +\left|\mathrm{v}_{3}\right| \frac{\left|\left(\mathbb{G}\left(\mathfrak{t}^{*}\right)-\mathbb{G}(a)\right)^{p+r}-(\mathbb{G}(\mathfrak{t})-\mathbb{G}(a))^{p+r}\right|}{\Gamma(p+r+1)} \\
& +\frac{\tilde{H}}{\Gamma(p+r+k+1)}\left[\mid\left(\mathbb{G}\left(\mathfrak{t}^{*}\right)-\mathbb{G}(a)\right)^{p+r+k}\right.
\end{aligned}
$$




$$
\left.-(\mathbb{G}(\mathfrak{t})-\mathbb{G}(a))^{p+r+k} \mid+2\left(\mathbb{G}\left(\mathfrak{t}^{*}\right)-\mathbb{G}(\mathfrak{t})\right)^{p+r+k}\right]
$$

Again, the right-hand side of (31) goes to zero as $\mathfrak{t}^{*} \rightarrow \mathfrak{t}$ independently of v. Finally,

$$
\begin{aligned}
\left|{ }^{c} \mathcal{D}_{a^{+}}^{p ; \mathbb{G}}\left({ }^{c} \mathcal{D}_{a^{+}}^{q ; \mathbb{G}}(\Psi \mathrm{v})\right)\left(\mathfrak{t}^{*}\right)-{ }^{c} \mathcal{D}_{a^{+}}^{p ; \mathbb{G}}\left({ }^{c} \mathcal{D}_{a^{+}}^{q ; \mathbb{G}}(\Psi \mathrm{v})\right)(\mathfrak{t})\right| \\
\leq\left|\mathrm{v}_{3}\right| \frac{\left|\left(\mathbb{G}\left(\mathfrak{t}^{*}\right)-\mathbb{G}(a)\right)^{r}-(\mathbb{G}(\mathfrak{t})-\mathbb{G}(a))^{r}\right|}{\Gamma(r+1)} \\
\quad+\left|\mathcal{I}_{a^{+}}^{r+k ; \mathbb{G}} h_{\mathrm{v}}\left(\mathfrak{t}^{*}\right)-\mathcal{I}_{a^{+}}^{r+k ; \mathbb{G}} h_{\mathrm{v}}(\mathfrak{t})\right| \\
\leq \frac{\tilde{H}}{\Gamma(r+k+1)}\left[\mid\left(\mathbb{G}\left(\mathfrak{t}^{*}\right)-\mathbb{G}(a)\right)^{r+k}\right. \\
\left.\quad-(\mathbb{G}(\mathfrak{t})-\mathbb{G}(a))^{r+k} \mid+2\left(\mathbb{G}\left(\mathfrak{t}^{*}\right)-\mathbb{G}(\mathfrak{t})\right)^{r+k}\right]
\end{aligned}
$$

and

$$
\begin{aligned}
& \left|{ }^{c} \mathcal{D}_{a^{+}}^{r ; \mathbb{G}}\left({ }^{c} \mathcal{D}_{a^{+}}^{p ; \mathbb{G}}\left({ }^{c} \mathcal{D}_{a^{+}}^{q ; \mathbb{G}}(\Psi \mathrm{v})\right)\right)\left(\mathfrak{t}^{*}\right)-{ }^{c} \mathcal{D}_{a^{+}}^{r ; \mathbb{G}}\left({ }^{c} \mathcal{D}_{a^{+}}^{p ; \mathbb{G}}\left({ }^{c} \mathcal{D}_{a^{+}}^{q ; \mathfrak{G}}(\Psi \mathrm{v})\right)\right)(\mathfrak{t})\right| \\
& \leq \frac{\tilde{H}}{\Gamma(k+1)}\left[\left|\left(\mathbb{G}\left(\mathfrak{t}^{*}\right)-\mathbb{G}(a)\right)^{k}-(\mathbb{G}(\mathfrak{t})-\mathbb{G}(a))^{k}\right|\right. \\
& \left.\quad+2\left(\mathbb{G}\left(\mathfrak{t}^{*}\right)-\mathbb{G}(\mathfrak{t})\right)^{k}\right],
\end{aligned}
$$

which independent of $v$. The right-hand sides of (34) and (33) approach 0 as $\mathfrak{t}^{*} \rightarrow \mathfrak{t}$. Therefore relations (30), (31), (32), and (34) imply that

$$
\left\|(\Psi \mathrm{v})\left(t^{*}\right)-(\Psi \mathrm{v})(\mathfrak{t})\right\| \rightarrow 0
$$

as $\mathfrak{t}^{*} \rightarrow \mathfrak{t}$. Thus the equicontinuity of $\Psi$ is confirmed. Hence $\Psi$ is compact on $N_{\epsilon}$ by the Arzelá-Ascoli theorem. Until now, we saw that the hypotheses of Theorem 2.5 are fulfilled for the operator $\Psi$. Thus one of two cases (i) or (ii) is valid. By (C3) we build

$$
\mathbb{U}:=\{\mathrm{v} \in C([a, b], \mathbb{R}):\|\mathrm{v}\|<B\}
$$

for $B>0$ via $\Lambda+\mathcal{O} \varrho_{0}^{*} f(B)<B$. With the help of (C2), by (29) we write

$$
\|\Psi \mathrm{v}\| \leq \Lambda+\mathcal{O} \varrho_{0}^{*} f(\|\mathrm{v}\|)
$$

Now we assume the existence of $\mathrm{v} \in \partial \mathbb{U}$ and $\mu \in(0,1)$ subject to $\mathrm{v}=\mu \Psi \mathrm{v}$. For such a selection of $\mathrm{v}$ and $\mu$, we may write by (34) that

$$
B=\|\mathrm{v}\|=\mu\|\Psi \mathrm{v}\|<\Lambda+\mathcal{O} \varrho_{0}^{*} f(\|\mathrm{v}\|)=\Lambda+\mathcal{O} \varrho_{0}^{*} f(B)<B,
$$

a contradiction. Therefore case (ii) does not hold, and by Theorem $2.5 \Psi$ admits a fixed point in $\overline{\mathbb{U}}$, which is regarded as a solution of the fractional $\mathbb{G}$-snap system (4), and this concludes the proof. 


\section{Stability criterion}

In this part, we review the stability criterion in the context of the Ulam-Hyers stability, its generalized version along with Ulam-Hyers-Rassias stability, and its generalized version for solutions of the fractional $\mathbb{G}$-snap system (4).

Definition 4.1 The fractional $\mathbb{G}$-snap BVP (4) is Ulam-Hyers stable if there exists $0<$ $c_{h}^{*} \in \mathbb{R}$ such that for all $\epsilon>0$ and $\mathrm{v}^{*} \in C([a, b], \mathbb{R})$ satisfying

$$
\left|{ }^{c} \mathcal{D}_{a^{+}}^{k ; \mathbb{G}}\left({ }^{c} \mathcal{D}_{a^{+}}^{r ; \mathbb{G}}\left({ }^{c} \mathcal{D}_{a^{+}}^{p ; \mathbb{G}}\left({ }^{c} \mathcal{D}_{a^{+}}^{q ; \mathbb{G}} \mathbf{v}^{*}(\mathfrak{t})\right)\right)\right)-\hat{h}_{\mathrm{v}^{*}}(\mathfrak{t})\right|<\epsilon,
$$

there exists $\mathrm{v} \in C([a, b], \mathbb{R})$ satisfying the fractional $\mathbb{G}$-snap BVP (4) with

$$
\left|\mathrm{v}^{*}(\mathfrak{t})-\mathrm{v}(\mathfrak{t})\right| \leq \epsilon c_{h}^{*} \quad \forall \mathfrak{t} \in[a, b]
$$

Definition 4.2 The fractional $\mathbb{G}$-snap BVP (4) is generalized Ulam-Hyers stable if there exists $c_{h}^{*} \in C\left(\mathbb{R}^{+}, \mathbb{R}^{+}\right)$with $c_{h}^{*}(0)=0$ such that for all $\epsilon>0$ and $\mathrm{v}^{*} \in C([a, b], \mathbb{R})$ satisfying the inequality

$$
\left|{ }^{c} \mathcal{D}_{a^{+}}^{k ; \mathfrak{G}}\left({ }^{c} \mathcal{D}_{a^{+}}^{r ; \mathbb{G}}\left({ }^{c} \mathcal{D}_{a^{+}}^{p ; \mathbb{G}}\left({ }^{c} \mathcal{D}_{a^{+}}^{q ; \mathbb{G}} \mathbf{v}^{*}(\mathfrak{t})\right)\right)\right)-\hat{h}_{\mathrm{v}^{*}}(\mathfrak{t})\right|<\epsilon
$$

there exists a solution $\mathrm{v} \in C([a, b], \mathbb{R})$ of the fractional $\mathbb{G}$-snap BVP (4) such that

$$
\left|\mathrm{v}^{*}(\mathfrak{t})-\mathrm{v}(\mathfrak{t})\right| \leq c_{h}^{*}(\epsilon) \quad \forall \mathfrak{t} \in[a, b]
$$

Definition 4.3 The fractional $\mathbb{G}$-snap BVP (4) is Ulam-Hyers-Rassias stable with respect to $\Phi$ if there exists $0<c_{h, \Phi}^{*} \in \mathbb{R}$ such that for all $\epsilon>0$ and $\mathrm{v}^{*} \in C([a, b], \mathbb{R})$ satisfying

$$
\left|{ }^{c} \mathcal{D}_{a^{+}}^{k ; \mathbb{G}}\left({ }^{c} \mathcal{D}_{a^{+}}^{r ; \mathbb{G}}\left({ }^{c} \mathcal{D}_{a^{+}}^{p ; \mathbb{G}}\left({ }^{c} \mathcal{D}_{a^{+}}^{q ; \mathbb{G}} \mathrm{v}^{*}(\mathfrak{t})\right)\right)\right)-\hat{h}_{\mathrm{v}^{*}}(\mathfrak{t})\right|<\epsilon \Phi(t),
$$

there exists a solution $\mathrm{v} \in C([a, b], \mathbb{R})$ of the fractional $\mathbb{G}$-snap BVP (4) such that

$$
\left|\mathrm{v}^{*}(\mathfrak{t})-\mathrm{v}(\mathfrak{t})\right| \leq \epsilon c_{h, \Phi}^{*} \Phi(t) \quad \forall \mathfrak{t} \in[a, b] .
$$

Definition 4.4 The fractional $\mathbb{G}$-snap BVP (4) is generalized Ulam-Hyers-Rassias stable with respect to $\Phi$ if there exists $0<c_{h, \Phi}^{*} \in \mathbb{R}$ such that for all $\mathrm{v}^{*} \in C([a, b], \mathbb{R})$ satisfying

$$
\left|{ }^{c} \mathcal{D}_{a^{+}}^{k ; \mathbb{G}}\left({ }^{c} \mathcal{D}_{a^{+}}^{r ; \mathbb{G}}\left({ }^{c} \mathcal{D}_{a^{+}}^{p ; \mathbb{G}}\left({ }^{c} \mathcal{D}_{a^{+}}^{q ; \mathbb{G}} \mathrm{v}^{*}(t)\right)\right)\right)-\hat{h}_{\mathrm{v}^{*}}(\mathfrak{t})\right|<\Phi(t),
$$

there exists a solution $\mathrm{v} \in C([a, b], \mathbb{R})$ of the fractional $\mathbb{G}$-snap BVP (4) such that

$$
\left|\mathrm{v}^{*}(\mathfrak{t})-\mathrm{v}(\mathfrak{t})\right| \leq c_{h, \Phi}^{*} \Phi(t) \quad \forall \mathfrak{t} \in[a, b]
$$

Remark 4.1 $\left(a_{1}\right)$ Def. 4.1 $\Rightarrow$ Def. 4.2; $\left(a_{2}\right)$ Def. $4.3 \Rightarrow$ Def. 4.4; and $\left(a_{3}\right)$ for $\Phi(\mathfrak{t})=1$, Def. $4.3 \Rightarrow$ Def. 4.1 . 
Remark 4.2 Note that $\mathrm{v}^{*} \in C([a, b], \mathbb{R})$ is called a solution ofinequality (35) iff there exists $g \in C([a, b], \mathbb{R})$ depending on $\mathrm{v}^{*}$ such that for all $\mathfrak{t} \in[a, b]$, (i) $|g(\mathfrak{t})|<\epsilon$; and (ii)

$$
{ }^{c} \mathcal{D}_{a^{+}}^{k ; \mathbb{G}}\left({ }^{c} \mathcal{D}_{a^{+}}^{r ; \mathbb{G}}\left({ }^{c} \mathcal{D}_{a^{+}}^{p ; \mathbb{G}}\left({ }^{c} \mathcal{D}_{a^{+}}^{q \mathbb{G}} \mathrm{V}^{*}(t)\right)\right)\right)=\hat{h}_{\mathrm{v}^{*}}(\mathfrak{t})+g(\mathfrak{t}) .
$$

Remark 4.3 Note that $\mathrm{v}^{*} \in C([a, b], \mathbb{R})$ is called a solution off inequality (36) iff there exists $g \in C([a, b], \mathbb{R})$ depending on $\mathrm{v}^{*}$ such that for all $\mathfrak{t} \in[a, b]$, (i) $|g(\mathfrak{t})|<\epsilon \Phi(\mathfrak{t})$; and (ii)

$$
{ }^{c} \mathcal{D}_{a^{+}}^{k ; \mathbb{G}}\left({ }^{c} \mathcal{D}_{a^{+}}^{r ; \mathbb{G}}\left({ }^{c} \mathcal{D}_{a^{+}}^{p ; \mathbb{G}}\left({ }^{c} \mathcal{D}_{a^{+}}^{q ; \mathbb{G}} \mathbf{V}^{*}(\mathfrak{t})\right)\right)\right)=\hat{h}_{\mathrm{v}^{*}}(\mathfrak{t})+g(\mathfrak{t}) .
$$

Here we discuss the Ulam-Hyers stability of the fractional $\mathbb{G}$-snap BVP (4).

Theorem 4.5 If all assumptions $(C 1)$ are fulfilled, then the fractional $\mathbb{G}$-snap BVP (4) is Ulam-Hyers stable on $[a, b]$ and is generalized Ulam-Hyers stable if $L \mathcal{O}<1$.

Proof For every $\epsilon>0$ and all $\mathrm{v}^{*} \in C([a, b], \mathbb{R})$ satisfying

$$
\left|{ }^{c} \mathcal{D}_{a^{+}}^{k ; \mathbb{G}}\left({ }^{c} \mathcal{D}_{a^{+}}^{r ; \mathbb{G}}\left({ }^{c} \mathcal{D}_{a^{+}}^{p ; \mathbb{G}}\left({ }^{c} \mathcal{D}_{a^{+}}^{q ; \mathbb{G}} \mathrm{v}(\mathfrak{t})\right)\right)\right)-\hat{h}_{\mathrm{v}}(\mathfrak{t})\right|<\epsilon
$$

we can find a function $g(\mathfrak{t})$ satisfying

$$
{ }^{c} \mathcal{D}_{a^{+}}^{k ; \mathbb{G}}\left({ }^{c} \mathcal{D}_{a^{+}}^{r, \mathbb{G}}\left({ }^{c} \mathcal{D}_{a^{+}}^{p ; \mathbb{G}}\left({ }^{c} \mathcal{D}_{a^{+}}^{q ; \mathbb{G}} \mathbf{v}(\mathfrak{t})\right)\right)\right)=\hat{h}_{\mathrm{v}}(\mathfrak{t})+g(\mathfrak{t})
$$

with $|g(\mathfrak{t})| \leq \epsilon$. It follows that

$$
\begin{aligned}
\mathrm{v}^{*}(\mathfrak{t})= & \mathrm{v}_{0}+\frac{\mathrm{v}_{1}(\mathbb{G}(\mathfrak{t})-\mathbb{G}(a))^{q}}{\Gamma(q+1)}+\frac{\mathrm{v}_{2}(\mathbb{G}(\mathfrak{t})-\mathbb{G}(a))^{p+q}}{\Gamma(p+q+1)} \\
& +\frac{\mathrm{v}_{3}(\mathbb{G}(\mathfrak{t})-\mathbb{G}(a))^{r+p+q}}{\Gamma(r+p+q+1)}+\mathcal{I}_{a^{+}}^{q+p+r+k ; \mathbb{G}} g(\mathfrak{t})+\mathcal{I}_{a^{+}}^{q+p+r+k ; \mathbb{G}} \hat{h}_{\mathrm{v}}(\mathfrak{t}) .
\end{aligned}
$$

Let $\mathrm{v} \in C([a, b], \mathbb{R})$ be the unique solution of the fractional $\mathbb{G}$-snap BVP $(4)$. Then it is given by

$$
\begin{aligned}
\mathrm{v}(\mathfrak{t})= & \mathrm{v}_{0}+\frac{\mathrm{v}_{1}(\mathbb{G}(\mathfrak{t})-\mathbb{G}(a))^{q}}{\Gamma(q+1)}+\frac{\mathrm{v}_{2}(\mathbb{G}(\mathfrak{t})-\mathbb{G}(a))^{p+q}}{\Gamma(p+q+1)} \\
& +\frac{\mathrm{v}_{3}(\mathbb{G}(\mathfrak{t})-\mathbb{G}(a))^{r+p+q}}{\Gamma(r+p+q+1)}+\mathcal{I}_{a^{+}}^{q+p+r+k ; \mathbb{G}} \hat{h}_{\mathrm{v}}(\mathfrak{t})
\end{aligned}
$$

and

$$
\begin{aligned}
\left|\mathrm{v}^{*}(\mathfrak{t})-\mathrm{v}(\mathfrak{t})\right| & \leq \mathcal{I}_{a^{+}}^{q+p+r+k ; \mathbb{G}}|g(\mathfrak{t})|+\mathcal{I}_{a^{+}}^{q+p+r+k ; \mathbb{G}}\left|\hat{h}_{\mathrm{v}^{*}}(\mathfrak{t})-\hat{h}_{\mathrm{v}}(\mathfrak{t})\right| \\
& \leq \frac{\epsilon(\mathbb{G}(b)-\mathbb{G}(a))^{q+p+r+k}}{\Gamma(q+p+r+k+1)}+\frac{L(\mathbb{G}(b)-\mathbb{G}(a))^{q+p+r+k}}{\Gamma(q+p+r+k+1)}\left\|\mathrm{v}^{*}-\mathrm{v}\right\| .
\end{aligned}
$$

Also,

$$
\left|\left({ }^{c} \mathcal{D}_{a^{+}}^{q ; \mathbb{G}} \mathrm{v}^{*}\right)(\mathfrak{t})-\left({ }^{c} \mathcal{D}_{a^{+}}^{q ; \mathfrak{G}} \mathrm{v}\right)(\mathfrak{t})\right|
$$




$$
\begin{aligned}
& \leq \mathcal{I}_{a^{+}}^{p+r+k ; \mathbb{G}}|g(\mathfrak{t})|+\mathcal{I}_{a^{+}}^{p+r+k ; \mathbb{G}}\left|\hat{h}_{\mathrm{v}^{*}}(\mathfrak{t})-\hat{h}_{\mathrm{v}}(\mathfrak{t})\right| \\
& \leq \frac{\epsilon(\mathbb{G}(b)-\mathbb{G}(a))^{p+r+k}}{\Gamma(p+r+k+1)}+\frac{L(\mathbb{G}(b)-\mathbb{G}(a))^{p+r+k}}{\Gamma(p+r+k+1)}\left\|\mathrm{v}^{*}-\mathrm{v}\right\|, \\
&\left|{ }^{c} \mathcal{D}_{a^{+}}^{p ; \mathbb{G}}\left({ }^{c} \mathcal{D}_{a^{+}}^{q ; \mathbb{G}} \mathrm{v}^{*}\right)(\mathfrak{t})-{ }^{c} \mathcal{D}_{a^{+}}^{p ; \mathbb{G}}\left({ }^{c} \mathcal{D}_{a^{+}}^{q ; \mathbb{G}} \mathrm{v}\right)(\mathfrak{t})\right| \\
& \leq \mathcal{I}_{a^{+}}^{r+k ; \mathbb{G}}|g(\mathfrak{t})|+\mathcal{I}_{a^{+}}^{r+k ; \mathbb{G}}\left|\hat{h}_{\mathrm{v}^{*}}(\mathfrak{t})-\hat{h}_{\mathrm{v}}(\mathfrak{t})\right| \\
& \leq \frac{\epsilon(\mathbb{G}(b)-\mathbb{G}(a))^{r+k}}{\Gamma(r+k+1)}+\frac{L(\mathbb{G}(b)-\mathbb{G}(a))^{r+k}}{\Gamma(r+k+1)}\left\|\mathrm{v}^{*}-\mathrm{v}\right\|,
\end{aligned}
$$

and

$$
\begin{aligned}
& \left|{ }^{c} \mathcal{D}_{a^{+}}^{r ; \mathbb{G}}\left({ }^{c} \mathcal{D}_{a^{+}}^{p ; \mathbb{G}}\left({ }^{c} \mathcal{D}_{a^{+}}^{q ; \mathfrak{G}} \mathrm{v}^{*}\right)(\mathfrak{t})\right)-{ }^{c} \mathcal{D}_{a^{+}}^{r ; \mathbb{G}}\left({ }^{c} \mathcal{D}_{a^{+}}^{p ; \mathbb{G}}\left({ }^{c} \mathcal{D}_{a^{+}}^{q ; \mathfrak{G}} \mathrm{v}\right)\right)(\mathfrak{t})\right| \\
& \quad \leq \mathcal{I}_{a^{+}}^{k ; \mathbb{G}}|g(\mathfrak{t})|+\mathcal{I}_{a^{+}}^{k \mathbb{G}}\left|\hat{h}_{\mathrm{v}^{*}}(\mathfrak{t})-\hat{h}_{\mathrm{v}}(\mathfrak{t})\right| \\
& \quad \leq \frac{\epsilon(\mathbb{G}(b)-\mathbb{G}(a))^{k}}{\Gamma(k+1)}+\frac{L(\mathbb{G}(b)-\mathbb{G}(a))^{k}}{\Gamma(k+1)}\left\|\mathrm{v}^{*}-\mathrm{v}\right\| .
\end{aligned}
$$

From (37), (38), (39), and (40) we get

$$
\begin{aligned}
\left\|\mathrm{v}^{*}-\mathrm{v}\right\|= & \sup _{\mathfrak{t} \in[a, b]}\left(\left|\mathrm{v}^{*}(\mathfrak{t})-\mathrm{v}(\mathfrak{t})\right|+\left|\left({ }^{c} \mathcal{D}_{a^{+}}^{q ; \mathbb{G}} \mathrm{v}^{*}\right)(\mathfrak{t})-\left({ }^{c} \mathcal{D}_{a^{+}}^{q ; \mathbb{G}} \mathrm{v}\right)(\mathfrak{t})\right|\right. \\
& +\left|{ }^{c} \mathcal{D}_{a^{+}}^{p ; \mathbb{G}}\left({ }^{c} \mathcal{D}_{a^{+}}^{q ; \mathbb{G}} \mathrm{v}^{*}\right)(\mathfrak{t})-{ }^{c} \mathcal{D}_{a^{+}}^{p ; \mathbb{G}}\left({ }^{c} \mathcal{D}_{a^{+}}^{q ; \mathbb{G}} \mathrm{v}\right)(\mathfrak{t})\right| \\
& \left.+\left|{ }^{c} \mathcal{D}_{a^{+}}^{r ; \mathbb{G}}\left({ }^{c} \mathcal{D}_{a^{+}}^{p ; \mathbb{G}}\left({ }^{c} \mathcal{D}_{a^{+}}^{q ; \mathbb{G}} \mathrm{v}^{*}\right)\right)(\mathfrak{t})-{ }^{c} \mathcal{D}_{a^{+}}^{r ; \mathbb{G}}\left({ }^{c} \mathcal{D}_{a^{+}}^{p ; \mathbb{G}}\left({ }^{c} \mathcal{D}_{a^{+}}^{q ; \mathbb{G}} \mathrm{v}\right)\right)(\mathfrak{t})\right|\right) \\
\leq & \mathcal{O} \epsilon+L \mathcal{O}\left\|\mathrm{v}^{*}-\mathrm{v}\right\|,
\end{aligned}
$$

where $\mathcal{O}$ is defined in (12). As a consequence, it follows that

$$
\left\|\mathrm{v}^{*}-\mathrm{v}\right\| \leq \frac{\mathcal{O} \epsilon}{1-L \mathcal{O}}
$$

If we let $c_{h}^{*}=\frac{\mathcal{O}}{1-L \mathcal{O}}$, then the Ulam-Hyers stability is fulfilled. Next, for

$$
c_{h}^{*}(\epsilon)=\frac{\mathcal{O}}{1-L \mathcal{O}} \epsilon
$$

with $c_{h}^{*}(0)=0$, the generalized Ulam-Hyers stability is fulfilled.

The Ulam-Hyers-Rassias stability for the fractional $\mathbb{G}$-snap BVP (4) is checked in the following:

\section{Theorem 4.6 Let conditions (C1) be satisfied, and assume that}

(C4) there exist an increasing map $\Phi \in C\left([a, b], \mathbb{R}^{+}\right)$and $\lambda_{\Phi}>0$ such that for all $\mathfrak{t} \in[a, b]$,

$$
\mathcal{I}_{a^{+}}^{q+p+r+k ; \mathbb{G}} \Phi(\mathfrak{t})+\mathcal{I}_{a^{+}}^{p+r+k ; \mathbb{G}} \Phi(\mathfrak{t})+\mathcal{I}_{a^{+}}^{r+k+; \mathbb{G}} \Phi(\mathfrak{t})+\mathcal{I}_{a^{+}}^{k ; \mathbb{G}} \Phi(\mathfrak{t})<\lambda_{\Phi} \Phi(\mathfrak{t}) .
$$

Then the fractional $\mathbb{G}$-snap BVP (4) is Ulam-Hyers-Rassias stable and is generalized Ulam-Hyers-Rassias stable. 
Proof For every $\epsilon>0$ and all $\mathrm{v}^{*} \in C([a, b], \mathbb{R})$ satisfying

$$
\left|{ }^{c} \mathcal{D}_{a^{+}}^{k ; \mathbb{G}}\left({ }^{c} \mathcal{D}_{a^{+}}^{r ; \mathbb{G}}\left({ }^{c} \mathcal{D}_{a^{+}}^{p ; \mathbb{G}}\left({ }^{c} \mathcal{D}_{a^{+}}^{q ; \mathbb{G}} \mathrm{v}(\mathfrak{t})\right)\right)\right)-\hat{h}_{\mathrm{v}}(\mathfrak{t})\right|<\epsilon \Phi(\mathfrak{t}),
$$

we can find a function $g(\mathfrak{t})$ satisfying

$$
{ }^{c} \mathcal{D}_{a^{+}}^{k ; \mathbb{G}}\left({ }^{c} \mathcal{D}_{a^{+}}^{r: \mathbb{G}}\left({ }^{c} \mathcal{D}_{a^{+}}^{p ; \mathbb{G}}\left({ }^{c} \mathcal{D}_{a^{+}}^{q ; \mathfrak{G}} \mathrm{v}(\mathfrak{t})\right)\right)\right)=\hat{h}_{\mathrm{v}}(\mathfrak{t})+g(\mathfrak{t})
$$

with $|g(\mathfrak{t})| \leq \epsilon \Phi(\mathfrak{t})$. It follows that

$$
\begin{aligned}
\mathrm{v}^{*}(\mathfrak{t})= & \mathrm{v}_{0}+\frac{\mathrm{v}_{1}(\mathbb{G}(\mathfrak{t})-\mathbb{G}(a))^{q}}{\Gamma(q+1)}+\frac{\mathrm{v}_{2}(\mathbb{G}(\mathfrak{t})-\mathbb{G}(a))^{p+q}}{\Gamma(p+q+1)} \\
& +\frac{\mathrm{v}_{3}(\mathbb{G}(\mathfrak{t})-\mathbb{G}(a))^{p+q+r}}{\Gamma(p+q+r+1)}+\mathcal{I}_{a^{+}}^{q+p+r+k ; \mathbb{G}} g(\mathfrak{t})+\mathcal{I}_{a^{+}}^{q+p+r+k ; \mathbb{G}} \hat{h}_{\mathrm{v}^{*}}(\mathfrak{t}) .
\end{aligned}
$$

If $\mathbf{v} \in C([a, b], \mathbb{R})$ is a unique solution of (4), then we have

$$
\begin{aligned}
\mathrm{v}(\mathfrak{t})= & \mathrm{v}_{0}+\frac{\mathrm{v}_{1}(\mathbb{G}(\mathfrak{t})-\mathbb{G}(a))^{q}}{\Gamma(q+1)}+\frac{\mathrm{v}_{2}(\mathbb{G}(\mathfrak{t})-\mathbb{G}(a))^{p+q}}{\Gamma(p+q+1)} \\
& +\frac{\mathrm{v}_{3}(\mathbb{G}(\mathfrak{t})-\mathbb{G}(a))^{p+q+r}}{\Gamma(p+q+r+1)}+\mathcal{I}_{a^{+}}^{p+q+r+k ; \mathbb{G}} \hat{h}_{\mathrm{v}}(\mathfrak{t}) .
\end{aligned}
$$

Then

$$
\begin{aligned}
\left|\mathrm{v}^{*}(\mathfrak{t})-\mathrm{v}(\mathfrak{t})\right| & \leq \mathcal{I}_{a^{+}}^{q+p+r+k ; \mathbb{G}}|g(\mathfrak{t})|+\mathcal{I}_{a^{+}}^{q+p+r+k ; \mathbb{G}}\left|\hat{h}_{\mathrm{v}^{*}}(\mathfrak{t})-\hat{h}_{\mathrm{v}}(\mathfrak{t})\right| \\
& \leq \epsilon \mathcal{I}_{a^{+}}^{q+p+r+k ; \mathbb{G}} \Phi(\mathfrak{t})+\frac{L(\mathbb{G}(b)-\mathbb{G}(a))^{q+p+r+k}}{\Gamma(q+p+r+k+1)}\left\|\mathrm{v}^{*}-\mathrm{v}\right\| .
\end{aligned}
$$

Also,

$$
\begin{aligned}
& \left|\left({ }^{c} \mathcal{D}_{a^{+}}^{q ; \mathfrak{G}} \mathrm{v}^{*}\right)(\mathfrak{t})-\left({ }^{c} \mathcal{D}_{a^{+}}^{q ; \mathfrak{G}} \mathrm{v}\right)(\mathfrak{t})\right| \\
& \quad \leq \mathcal{I}_{a^{+}}^{p+r+k ; \mathbb{G}}|g(\mathfrak{t})|+\mathcal{I}_{a^{+}}^{p+r+k ; \mathbb{G}}\left|\hat{h}_{\mathrm{v}^{*}}(\mathfrak{t})-\hat{h}_{\mathrm{v}}(\mathfrak{t})\right| \\
& \quad \leq \epsilon \mathcal{I}_{a^{+}}^{p+r+k ; \mathbb{G}} \Phi(\mathfrak{t})+\frac{L(\mathbb{G}(b)-\mathbb{G}(a))^{p+r+k}}{\Gamma(p+r+k+1)}\left\|\mathrm{v}^{*}-\mathrm{v}\right\|, \\
& \left|{ }^{c} \mathcal{D}_{a^{+}}^{p ; \mathbb{G}}\left({ }^{c} \mathcal{D}_{a^{+}}^{q ; \mathbb{G}} \mathrm{v}^{*}\right)(\mathfrak{t})-{ }^{c} \mathcal{D}_{a^{+}}^{p ; \mathbb{G}}\left({ }^{c} \mathcal{D}_{a^{+}}^{q ; \mathbb{G}} \mathrm{v}\right)(\mathfrak{t})\right| \\
& \quad \leq \mathcal{I}_{a^{+}}^{r+k ; \mathbb{G}}|g(\mathfrak{t})|+\mathcal{I}_{a^{+}}^{r+k ; \mathbb{G}}\left|\hat{h}_{\mathrm{v}^{*}}(\mathfrak{t})-\hat{h}_{\mathrm{v}}(\mathfrak{t})\right| \\
& \quad \leq \epsilon \mathcal{I}_{a^{+}}^{r+k ; \mathbb{G}} \Phi(\mathfrak{t})+\frac{L(\mathbb{G}(b)-\mathbb{G}(a))^{r+k}}{\Gamma(r+k+1)}\left\|\mathrm{v}^{*}-\mathrm{v}\right\|,
\end{aligned}
$$

and

$$
\begin{aligned}
& \left|{ }^{c} \mathcal{D}_{a^{+}}^{r ; \mathbb{G}}\left({ }^{c} \mathcal{D}_{a^{+}}^{p ; \mathbb{G}}\left({ }^{c} \mathcal{D}_{a^{+}}^{q ; \mathfrak{G}} \mathrm{v}^{*}\right)\right)(\mathfrak{t})-{ }^{c} \mathcal{D}_{a^{+}}^{r ; \mathbb{G}}\left({ }^{c} \mathcal{D}_{a^{+}}^{p ; \mathbb{G}}\left({ }^{c} \mathcal{D}_{a^{+}}^{q ; \mathbb{G}} \mathrm{v}\right)\right)(\mathfrak{t})\right| \\
& \quad \leq \mathcal{I}_{a^{+}}^{k ; \mathbb{G}}|g(\mathfrak{t})|+\mathcal{I}_{a^{+}}^{k ; \mathbb{G}}\left|\hat{h}_{\mathrm{v}^{*}}(\mathfrak{t})-\hat{h}_{\mathrm{v}}(\mathfrak{t})\right| \\
& \quad \leq \epsilon \mathcal{I}_{a^{+}}^{k ; \mathbb{G}} \Phi(\mathfrak{t})+\frac{L(\mathbb{G}(b)-\mathbb{G}(a))^{r+k}}{\Gamma(k+1)}\left\|\mathrm{v}^{*}-\mathrm{v}\right\| .
\end{aligned}
$$


From (42), (43), (44), and (45) we get

$$
\begin{aligned}
\left\|\mathrm{v}^{*}-\mathrm{v}\right\|= & \sup _{\mathfrak{t} \in[a, b]}\left(\left|\mathrm{v}^{*}(\mathfrak{t})-\mathrm{v}(\mathfrak{t})\right|+\left|\left({ }^{c} \mathcal{D}_{a^{+}}^{q ; \mathfrak{G}} \mathrm{v}^{*}\right)(t)-\left({ }^{c} \mathcal{D}_{a^{+}}^{q ; \mathbb{G}} \mathrm{v}\right)(\mathfrak{t})\right|\right. \\
& +\left|{ }^{c} \mathcal{D}_{a^{+}}^{p ; \mathbb{G}}\left({ }^{c} \mathcal{D}_{a^{+}}^{q ; \mathbb{G}} \mathrm{v}^{*}\right)(\mathfrak{t})-{ }^{c} \mathcal{D}_{a^{+}}^{p ; \mathbb{G}}\left({ }^{c} \mathcal{D}_{a^{+}}^{q ; \mathbb{G}} \mathrm{v}\right)(\mathfrak{t})\right| \\
& \left.+\left|{ }^{c} \mathcal{D}_{a^{+}}^{r ; \mathbb{G}}\left({ }^{c} \mathcal{D}_{a^{+}}^{p ; \mathbb{G}}\left({ }^{c} \mathcal{D}_{a^{+}}^{q ; \mathbb{G}} \mathrm{v}^{*}\right)\right)(\mathfrak{t})-{ }^{c} \mathcal{D}_{a^{+}}^{r, \mathbb{G}}\left({ }^{c} \mathcal{D}_{a^{+}}^{p ; \mathbb{G}}\left({ }^{c} \mathcal{D}_{a^{+}}^{q ; \mathbb{G}} \mathrm{v}\right)\right)(\mathfrak{t})\right|\right) \\
\leq & \epsilon\left[\mathcal{I}_{a^{+}}^{q+p+r+r ; \mathfrak{G}} \Phi(\mathfrak{t})+\mathcal{I}_{a^{+}}^{p+r+k ; \mathbb{G}} \Phi(\mathfrak{t})+\mathcal{I}_{a^{+}}^{r+k ; \mathbb{G}} \Phi(\mathfrak{t})\right. \\
& \left.+\mathcal{I}_{a^{+}}^{k ; \mathbb{G}} \Phi(\mathfrak{t})\right]+L \mathcal{O}\left\|\mathrm{v}^{*}-\mathrm{v}\right\| \\
\leq & \epsilon \lambda_{\Phi} \Phi(\mathfrak{t})+L \mathcal{O}\left\|\mathrm{v}^{*}-\mathrm{v}\right\|,
\end{aligned}
$$

where $\mathcal{O}$ is defined in (12). Accordingly, it gives

$$
\left\|\mathrm{v}^{*}-\mathrm{v}\right\| \leq \frac{\epsilon \lambda_{\Phi} \Phi(\mathfrak{t})}{1-L \mathcal{O}}
$$

If we let $c_{h, \Phi}^{*}=\frac{\lambda_{\Phi}}{1-L \mathcal{O}}$, then the fractional $\mathbb{G}$-snap BVP (4) is stable in the Ulam-HyersRassias sense. Along with this, setting $\epsilon=1$, the fractional $\mathbb{G}$-snap BVP (4) is generalized Ulam-Hyers-Rassias stable.

\section{Inclusion version of (4)}

Here we will derive the existence of solutions to the inclusion version of fractional nonlinear snap system of the $\mathbb{G}$-Caputo sense with initial conditions (4), which takes the form

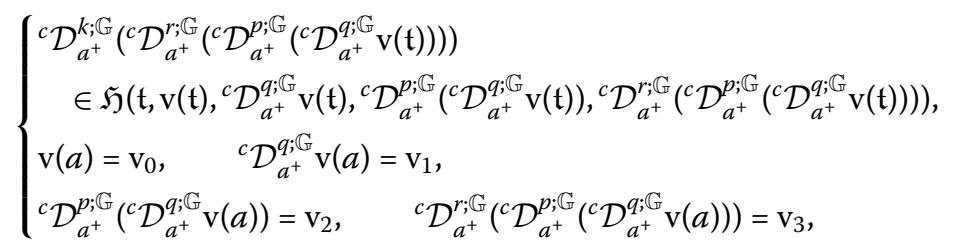

where $\mathfrak{H}$ ia a multifunction on the product space $[a, b] \times \mathbb{R}^{4}$. The function $\mathrm{v} \in \mathcal{C}:=$ $C([a, b], \mathbb{R})$ is called a solution of system $(46)$ if it satisfies the boundary conditions and there is $\wp \in L^{1}([a, b])$ such that $\wp(\mathfrak{t}) \in \widehat{\mathfrak{H}}_{\mathrm{v}}(\mathfrak{t})$ for almost all $\mathfrak{t} \in[a, b]$, where

$$
\widehat{\mathfrak{H}}_{\mathrm{v}}(\mathfrak{t})=\mathfrak{H}\left(\mathfrak{t}, \mathrm{v}(\mathfrak{t}),{ }^{c} \mathcal{D}_{a^{+}}^{q ; \mathfrak{G}} \mathrm{v}(\mathfrak{t}),{ }^{c} \mathcal{D}_{a^{+}}^{p ; \mathbb{G}}\left({ }^{c} \mathcal{D}_{a^{+}}^{q ; \mathbb{G}} \mathrm{v}(\mathfrak{t})\right),{ }^{c} \mathcal{D}_{a^{+}}^{r ; \mathbb{G}}\left({ }^{c} \mathcal{D}_{a^{+}}^{p ; \mathbb{G}}\left({ }^{c} \mathcal{D}_{a^{+}}^{q ; \mathbb{G}} \mathrm{v}(\mathfrak{t})\right)\right)\right)
$$

and

$$
\begin{aligned}
\mathrm{v}(\mathfrak{t})= & \mathrm{v}_{0}+\frac{\mathrm{v}_{1}(\mathbb{G}(\mathfrak{t})-\mathbb{G}(a))^{q}}{\Gamma(q+1)}+\frac{\mathrm{v}_{2}(\mathbb{G}(\mathfrak{t})-\mathbb{G}(a))^{q+p}}{\Gamma(q+p+1)} \\
& +\frac{\mathrm{v}_{3}(\mathbb{G}(\mathfrak{t})-\mathbb{G}(a))^{q+p+r}}{\Gamma(q+p+r+1)} \\
& +\int_{a}^{\mathfrak{t}} \mathbb{G}^{\prime}(\xi) \frac{(\mathbb{G}(\mathfrak{t})-\mathbb{G}(\xi))^{q+p+r+k-1}}{\Gamma(q+p+r+k)} \wp(\xi) \mathrm{d} \xi
\end{aligned}
$$

for all $\mathfrak{t} \in[a, b]$. For each $\mathrm{v} \in \mathcal{C}$, we define the set of selections of the operator $\mathfrak{H}$ as

$$
\mathfrak{S}_{\mathfrak{H}, \mathrm{v}}=\left\{\wp \in L^{1}([a, b]): \wp(\mathfrak{t}) \in \widehat{\mathfrak{H}}_{\mathrm{v}}(\mathfrak{t}), \forall \mathfrak{t} \in[a, b]\right\}
$$


and define the operator $\mathfrak{U}: \mathcal{C} \rightarrow \mathcal{P}(\mathcal{C})$ by

$$
\mathfrak{U}(\mathrm{v})=\left\{\mathfrak{p} \in \mathcal{C}: \text { there exists } \wp \in \mathfrak{S}_{\mathfrak{H}, \mathrm{v}} \text { such thatp }(\mathfrak{t})=\Upsilon(\mathfrak{t}) \forall \mathfrak{t} \in[a, b]\right\}
$$

where

$$
\begin{aligned}
\Upsilon(\mathfrak{t})= & \mathrm{v}_{0}+\frac{\mathrm{v}_{1}(\mathbb{G}(\mathfrak{t})-\mathbb{G}(a))^{q}}{\Gamma(q+1)}+\frac{\mathrm{v}_{2}(\mathbb{G}(\mathfrak{t})-\mathbb{G}(a))^{q+p}}{\Gamma(q+p+1)} \\
& +\frac{\mathrm{v}_{3}(\mathbb{G}(\mathfrak{t})-\mathbb{G}(a))^{q+p+r}}{\Gamma(q+p+r+1)} \\
& +\int_{a}^{\mathfrak{t}} \mathbb{G}^{\prime}(\xi) \frac{(\mathbb{G}(\mathfrak{t})-\mathbb{G}(\xi))^{q+p+r+k-1}}{\Gamma(q+p+r+k)} \wp(\xi) \mathrm{d} \xi .
\end{aligned}
$$

Theorem 5.1 Let $\mathfrak{H}:[a, b] \times \mathcal{C}^{4} \rightarrow \mathcal{P}_{\mathrm{CP}}(\mathcal{C})$ be a multifunction. Suppose that the following conditions are satisfied:

(C5) The multifunction $\mathfrak{H}$ is integrable and bounded, and

$$
\mathfrak{H}\left(\cdot, \mathrm{v}_{1}, \mathrm{v}_{2}, \mathrm{v}_{3}, \mathrm{v}_{4}\right):[a, b] \rightarrow \mathcal{P}_{\mathrm{CP}}(\mathcal{C})
$$

is measurable for $\mathrm{v}_{1}, \mathrm{v}_{2}, \mathrm{v}_{3}, \mathrm{v}_{4} \in \mathcal{C}$;

(C6) There exist $\phi \in C([a, b],[0, \infty))$ and a nondecreasing function $\psi \in \Pi$ such that

$$
\mathcal{H}_{d}\left(\mathfrak{H}\left(\mathfrak{t}, \mathrm{v}_{1}, \mathrm{v}_{2}, \mathrm{v}_{3}, \mathrm{v}_{4}\right), \mathfrak{H}\left(\mathfrak{t}, \mathbf{v}_{1}, \mathbf{v}_{2}, \mathbf{v}_{3}, \mathbf{v}_{4}\right)\right) \leq \frac{\phi(\mathfrak{t}) \lambda^{*}}{\|\phi\|} \psi\left(\sum_{k=1}^{4}\left|\mathrm{v}_{k}-\mathbf{v}_{k}^{\prime}\right|\right)
$$

for all $\mathrm{t} \in[a, b]$ and $\mathrm{v}_{1}, \mathrm{v}_{2}, \mathrm{v}_{3}, \mathrm{v}_{4}, \dot{v}_{1}^{\prime}, \mathbf{v}_{2}, \dot{v}_{3}, \mathbf{v}_{4} \in \mathcal{C}$, where $\mathcal{O}^{*}=\mathcal{O}^{-1}$;

(C7) There is $\chi^{*}: \mathbb{R}^{4} \times \mathbb{R}^{4} \rightarrow \mathbb{R}$ such that

$$
\chi^{*}\left(\left(\mathrm{v}_{1}, \mathrm{v}_{2}, \mathrm{v}_{3}, \mathrm{v}_{4}\right),\left(\mathbf{v}_{1}^{\prime}, \mathbf{v}_{2}, \mathbf{v}_{3}, \mathbf{v}_{4}\right)\right) \geq 0
$$

for all $\mathbf{v}_{k}, \mathbf{v}_{k} \in \mathcal{C}(k=1,2,3,4)$;

(C8) If $\left\{\mathrm{v}_{n}\right\}$ is a sequence in $\mathcal{C}$ with $\mathrm{v}_{n} \rightarrow \mathrm{v}$ and

$$
\begin{aligned}
& \chi^{*}\left(\left(\mathrm{v}_{n}(\mathfrak{t}),{ }^{c} \mathcal{D}_{a^{+}}^{q ; \mathbb{G}} \mathbf{v}_{n}(\mathfrak{t}),{ }^{c} \mathcal{D}_{a^{+}}^{p ; \mathbb{G}}\left({ }^{c} \mathcal{D}_{a^{+}}^{q ; \mathbb{G}} \mathbf{v}_{n}(\mathfrak{t})\right),\right.\right. \\
& \left.{ }^{c} \mathcal{D}_{a^{+}}^{r ; \mathbb{G}}\left({ }^{c} \mathcal{D}_{a^{+}}^{p ; \mathbb{G}}\left({ }^{c} \mathcal{D}_{a^{+}}^{q ; \mathbb{G}} \mathbf{v}_{n}(\mathfrak{t})\right)\right)\right), \\
& \left(\mathrm{v}_{n+1}(\mathfrak{t}),{ }^{c} \mathcal{D}_{a^{+}}^{q ; \mathbb{G}} \mathbf{v}_{n+1}(\mathfrak{t}),{ }^{c} \mathcal{D}_{a^{+}}^{p ; \mathbb{G}}\left({ }^{c} \mathcal{D}_{a^{+}}^{q ; \mathbb{G}} \mathbf{v}_{n+1}(\mathfrak{t})\right),\right. \\
& \left.\left.{ }^{c} \mathcal{D}_{a^{+}}^{r ; \mathbb{G}}\left({ }^{c} \mathcal{D}_{a^{+}}^{p ; \mathbb{G}}\left({ }^{c} \mathcal{D}_{a^{+}}^{q ; \mathbb{G}} \mathbf{v}_{n+1}(\mathfrak{t})\right)\right)\right)\right) \geq 0
\end{aligned}
$$

for all $\mathfrak{t} \in[a, b]$ and natural numbers $n$, then there exists a subsequence $\left\{\mathrm{v}_{n_{j}}\right\}$ of $\left\{\mathrm{v}_{n}\right\}$ such that

$$
\begin{gathered}
\chi^{*}\left(\left(\mathrm{v}_{n_{j}}(\mathfrak{t}),{ }^{c} \mathcal{D}_{a^{+}}^{q ; \mathbb{G}} \mathrm{v}_{n_{j}}(\mathfrak{t}),{ }^{c} \mathcal{D}_{a^{+}}^{p ; \mathbb{G}}\left({ }^{c} \mathcal{D}_{a^{+}}^{q ; \mathbb{G}} \mathrm{v}_{n_{j}}(\mathfrak{t})\right),\right.\right. \\
\left.{ }^{c} \mathcal{D}_{a^{+}}^{r ; \mathbb{G}}\left({ }^{c} \mathcal{D}_{a^{+}}^{p ; \mathbb{G}}\left({ }^{c} \mathcal{D}_{a^{+}}^{q ; \mathbb{G}} \mathrm{V}_{n_{j}}(\mathfrak{t})\right)\right)\right), \\
\left(\mathrm{v}(\mathfrak{t}),{ }^{c} \mathcal{D}_{a^{+}}^{q ; \mathbb{G}} \mathrm{v}(\mathfrak{t}),{ }^{c} \mathcal{D}_{a^{+}}^{p ; \mathbb{G}}\left({ }^{c} \mathcal{D}_{a^{+}}^{q ; \mathbb{G}} \mathrm{v}(\mathfrak{t})\right),\right.
\end{gathered}
$$




$$
\left.\left.{ }^{c} \mathcal{D}_{a^{+}}^{r ; \mathbb{G}}\left({ }^{c} \mathcal{D}_{a^{+}}^{p ; \mathbb{G}}\left({ }^{c} \mathcal{D}_{a^{+}}^{q ; \mathbb{G}} \mathrm{v}(\mathfrak{t})\right)\right)\right)\right) \geq 0
$$

for all $\mathfrak{t} \in[a, b]$ and $j \geq 1$;

(C9) There exist $\mathrm{v}_{0} \in \mathcal{C}$ and $\mathfrak{p} \in \mathfrak{U}\left(\mathrm{v}_{0}\right)$ such that

$$
\begin{gathered}
\chi^{*}\left(\left(\left(\mathrm{v}_{0}(\mathfrak{t}),{ }^{c} \mathcal{D}_{a^{+}}^{q ; \mathbb{G}} \mathrm{v}_{0}(\mathfrak{t}),{ }^{c} \mathcal{D}_{a^{+}}^{p ; \mathbb{G}}\left({ }^{c} \mathcal{D}_{a^{+}}^{q ; \mathbb{G}} \mathrm{V}_{0}(\mathfrak{t})\right),\right.\right.\right. \\
\left.{ }^{c} \mathcal{D}_{a^{+}}^{r ; \mathfrak{G}}\left({ }^{c} \mathcal{D}_{a^{+}}^{p ; \mathbb{G}}\left({ }^{c} \mathcal{D}_{a^{+}}^{q ; \mathfrak{G}} \mathrm{v}_{0}(\mathfrak{t})\right)\right)\right), \\
\left(\mathfrak{p}(\mathfrak{t}),{ }^{c} \mathcal{D}_{a^{+}}^{q \mathbb{G}} \mathfrak{p}(\mathfrak{t}),{ }^{c} \mathcal{D}_{a^{+}}^{p ; \mathbb{G}}\left({ }^{c} \mathcal{D}_{a^{+}}^{q ; \mathbb{G}} \mathfrak{p}(\mathfrak{t})\right),\right. \\
\left.\left.{ }^{c} \mathcal{D}_{a^{+}}^{r ; \mathbb{G}}\left({ }^{c} \mathcal{D}_{a^{+}}^{p ; \mathbb{G}}\left({ }^{c} \mathcal{D}_{a^{+}}^{q ; \mathbb{G}} \mathfrak{p}(\mathfrak{t})\right)\right)\right)\right) \geq 0
\end{gathered}
$$

for $\mathfrak{t} \in[a, b]$, where $\mathfrak{U}: \mathcal{C} \rightarrow P(\mathcal{C})$ is defined by (48);

(C10) For any $\mathrm{v} \in \mathcal{C}$ and $\mathfrak{p} \in \mathfrak{U}(\mathrm{v})$ with

$$
\begin{aligned}
& \chi^{*}\left(\left(\mathrm{v}(\mathfrak{t}),{ }^{c} \mathcal{D}_{a^{+}}^{q ; \mathbb{G}} \mathrm{v}(\mathfrak{t}),{ }^{c} \mathcal{D}_{a^{+}}^{p ; \mathbb{G}}\left({ }^{c} \mathcal{D}_{a^{+}}^{q ; \mathbb{G}} \mathrm{v}(\mathfrak{t})\right),\right.\right. \\
& \left.{ }^{c} \mathcal{D}_{a^{+}}^{r ; \mathbb{G}}\left({ }^{c} \mathcal{D}_{a^{+}}^{p ; \mathbb{G}}\left({ }^{c} \mathcal{D}_{a^{+}}^{q ; \mathbb{G}} \mathrm{v}(\mathfrak{t})\right)\right)\right), \\
& \left(\mathfrak{p}(\mathfrak{t}),{ }^{c} \mathcal{D}_{a^{+}}^{q ; \mathbb{G}} \mathfrak{p}(\mathfrak{t}),{ }^{c} \mathcal{D}_{a^{+}}^{p ; \mathbb{G}}\left({ }^{c} \mathcal{D}_{a^{+}}^{q ; \mathbb{G}} \mathfrak{p}(\mathfrak{t})\right),\right. \\
& \left.\left.{ }^{c} \mathcal{D}_{a^{+}}^{r ; \mathbb{G}}\left({ }^{c} \mathcal{D}_{a^{+}}^{p: \mathbb{G}}\left({ }^{c} \mathcal{D}_{a^{+}}^{q ; \mathbb{G}} \mathfrak{p}(\mathfrak{t})\right)\right)\right)\right) \geq 0,
\end{aligned}
$$

there exists $\mathfrak{p}^{*} \in \mathfrak{U}(\mathrm{v})$ such that

$$
\begin{gathered}
\chi^{*}\left(\left(\mathfrak{p}(\mathfrak{t}),{ }^{c} \mathcal{D}_{a^{+}}^{q ; \mathbb{G}} \mathfrak{p}(\mathfrak{t}),{ }^{c} \mathcal{D}_{a^{+}}^{p ; \mathbb{G}}\left({ }^{c} \mathcal{D}_{a^{+}}^{q ; \mathbb{G}} \mathfrak{p}(\mathfrak{t})\right),\right.\right. \\
\left.{ }^{c} \mathcal{D}_{a^{+}}^{r ; \mathbb{G}}\left({ }^{c} \mathcal{D}_{a^{+}}^{p ; \mathbb{G}}\left({ }^{c} \mathcal{D}_{a^{+}}^{q ; \mathbb{G}} \mathfrak{p}(\mathfrak{t})\right)\right)\right), \\
\left(\mathrm{v}(\mathfrak{t}),{ }^{c} \mathcal{D}_{a^{+}}^{q ; \mathbb{G}} \mathfrak{p}^{*}(\mathfrak{t}),{ }^{c} \mathcal{D}_{a^{+}}^{p ; \mathbb{G}}\left({ }^{c} \mathcal{D}_{a^{+}}^{q ; \mathbb{G}} \mathfrak{p}^{*}(\mathfrak{t})\right),\right. \\
\left.\left.{ }^{c} \mathcal{D}_{a^{+}}^{r ; \mathbb{G}}\left({ }^{c} \mathcal{D}_{a^{+}}^{p ; \mathbb{G}}\left({ }^{c} \mathcal{D}_{a^{+}}^{q ; \mathbb{G}} \mathfrak{p}^{*}(\mathfrak{t})\right)\right)\right)\right) \geq 0
\end{gathered}
$$

for all $\mathfrak{t} \in[a, b]$.

Then the inclusion problem (46) has at least one solution.

Proof Obviously, the fixed point of $\mathfrak{U}: \mathcal{C} \rightarrow \mathcal{P}(\mathcal{C})$ is a solution of BVP (46). Since the multivalued map $\mathfrak{t} \rightarrow \widehat{\mathfrak{H}}_{\mathrm{v}}(\mathfrak{t})$ is closed-valued and measurable for all $\mathrm{v} \in \mathcal{C}, \mathfrak{H}$ has measurable selection, and $\mathfrak{S}_{\mathfrak{H}, \mathrm{v}}$ is nonempty. We have to prove that $\mathfrak{U}(\mathrm{v})$ is closed in $\mathcal{C}$ for $\mathrm{v} \in \mathcal{C}$. Take $\left\{\mathrm{v}_{n}\right\}$ in $\mathfrak{U}(\mathrm{v})$ such that $\mathrm{v}_{n} \rightarrow \mathrm{v}$. For each $n, \wp_{n} \in \mathfrak{S}_{\mathfrak{H}, \mathrm{v}}$ is chosen such that

$$
\begin{aligned}
\mathrm{v}_{n}(\mathfrak{t})= & \mathrm{v}_{0}+\frac{\mathrm{v}_{1}(\mathbb{G}(\mathfrak{t})-\mathbb{G}(a))^{q}}{\Gamma(q+1)}+\frac{\mathrm{v}_{2}(\mathbb{G}(\mathfrak{t})-\mathbb{G}(a))^{q+p}}{\Gamma(q+p+1)} \\
& +\frac{\mathrm{v}_{3}(\mathbb{G}(\mathfrak{t})-\mathbb{G}(a))^{q+p+r}}{\Gamma(q+p+r+1)} \\
& +\int_{a}^{\mathfrak{t}} \mathbb{G}^{\prime}(\xi) \frac{(\mathbb{G}(\mathfrak{t})-\mathbb{G}(\xi))^{q+p+r+k-1}}{\Gamma(q+p+r+k)} \wp_{n}(\xi) \mathrm{d} \xi
\end{aligned}
$$

for all $\mathfrak{t} \in[a, b]$. Since $\mathfrak{H}$ has compact values, we define a subsequence of $\left\{\wp_{n}\right\}$ (again by the same notation) that converges to $\wp \in L^{1}([0,1])$. Hence $\wp \in \mathfrak{S}_{\mathfrak{H}, \mathrm{v}}$ and

$$
\mathrm{v}_{n}(\mathfrak{t}) \rightarrow \mathrm{v}(\mathfrak{t})=\mathrm{v}_{0}+\frac{\mathrm{v}_{1}(\mathbb{G}(\mathfrak{t})-\mathbb{G}(a))^{q}}{\Gamma(q+1)}+\frac{\mathrm{v}_{2}(\mathbb{G}(\mathfrak{t})-\mathbb{G}(a))^{q+p}}{\Gamma(q+p+1)}
$$




$$
\begin{aligned}
& +\frac{\mathrm{v}_{3}(\mathbb{G}(\mathfrak{t})-\mathbb{G}(a))^{q+p+r}}{\Gamma(q+p+r+1)} \\
& +\int_{a}^{\mathfrak{t}} \mathbb{G}^{\prime}(\xi) \frac{(\mathbb{G}(\mathfrak{t})-\mathbb{G}(\xi))^{q+p+r+k-1}}{\Gamma(q+p+r+k)} \wp(\xi) \mathrm{d} \xi
\end{aligned}
$$

for all $\mathfrak{t} \in[a, b]$, which gives that $\mathrm{v} \in \mathfrak{U}(\mathrm{v})$ and $\mathfrak{U}$ is closed valued. As $\mathfrak{H}$ is compact-valued, it is a simple task to affirm the boundedness of $\mathfrak{U}(\mathrm{v})$ for arbitrary $\mathrm{v} \in \mathcal{C}$. We have to prove that $\mathfrak{U}$ is an $\alpha-\psi$-contraction. For such a goal, we define $\alpha(\mathrm{v}, \hat{\mathrm{v}})=1$ whenever

$$
\begin{gathered}
\chi^{*}\left(\left(\mathrm{v}(\mathfrak{t}),{ }^{c} \mathcal{D}_{a^{+}}^{q ; \mathbb{V}} \mathrm{v}(\mathfrak{t}),{ }^{c} \mathcal{D}_{a^{+}}^{p ; \mathbb{G}}\left({ }^{c} \mathcal{D}_{a^{+}}^{q ; \mathbb{G}} \mathrm{v}(\mathfrak{t})\right),\right.\right. \\
\left.{ }^{c} \mathcal{D}_{a^{+}}^{r ; \mathbb{G}}\left({ }^{c} \mathcal{D}_{a^{+}}^{p ; \mathbb{G}}\left({ }^{c} \mathcal{D}_{a^{+}}^{q ; \mathbb{G}} \mathrm{v}(\mathfrak{t})\right)\right)\right), \\
\left(\hat{\mathrm{v}}(\mathfrak{t}),{ }^{c} \mathcal{D}_{a^{+}}^{q ; \mathbb{V}}(\mathfrak{t}),{ }^{c} \mathcal{D}_{a^{+}}^{p ; \mathbb{G}}\left({ }^{c} \mathcal{D}_{a^{+}}^{q ; \mathbb{G}} \dot{\mathrm{v}}(\mathfrak{t})\right),\right. \\
\left.\left.{ }^{c} \mathcal{D}_{a^{+}}^{r ; \mathbb{G}}\left({ }^{c} \mathcal{D}_{a^{+}}^{p ; \mathbb{G}}\left({ }^{c} \mathcal{D}_{a^{+}}^{q ; \mathbb{G}} \dot{\mathrm{v}}(\mathfrak{t})\right)\right)\right)\right) \geq 0,
\end{gathered}
$$

otherwise $\alpha\left(\mathrm{v}, \hat{\mathrm{v}}^{\prime}\right)=0$ for all $\mathrm{v}, \hat{\mathrm{v}} \in \mathcal{C}$. Let $\mathrm{v}, \mathrm{v} \in \mathcal{C}$ and $\hbar_{1}^{*} \in \mathfrak{U}(\hat{\mathrm{v}})$ and choose $\wp_{1} \in \mathfrak{S}_{\mathfrak{H}, \mathrm{v}}$ such that

$$
\begin{aligned}
\hbar_{1}^{*}(\mathfrak{t})= & \mathrm{v}_{0}+\frac{\mathrm{v}_{1}(\mathbb{G}(\mathfrak{t})-\mathbb{G}(a))^{q}}{\Gamma(q+1)}+\frac{\mathrm{v}_{2}(\mathbb{G}(\mathfrak{t})-\mathbb{G}(a))^{q+p}}{\Gamma(q+p+1)} \\
& +\frac{\mathrm{v}_{3}(\mathbb{G}(\mathfrak{t})-\mathbb{G}(a))^{q+p+r}}{\Gamma(q+p+r+1)} \\
& +\int_{a}^{\mathfrak{t}} \mathbb{G}^{\prime}(\xi) \frac{(\mathbb{G}(\mathfrak{t})-\mathbb{G}(\xi))^{q+p+r+k-1}}{\Gamma(q+p+r+k)} \wp_{1}(\xi) \mathrm{d} \xi
\end{aligned}
$$

for all $\mathfrak{t} \in[a, b]$. We estimate

$$
\begin{aligned}
& \mathcal{H}_{d}\left(\widehat{\mathfrak{H}}_{\mathrm{v}}(\mathfrak{t}), \widehat{\mathfrak{H}}_{\dot{\mathrm{v}}}(\mathfrak{t})\right) \\
& \leq \frac{\phi(\mathfrak{t}) \mathcal{O}^{*}}{\|\phi\|} \psi\left(|\mathrm{v}-\hat{\mathrm{v}}|+\left|{ }^{c} \mathcal{D}_{a^{+}}^{q ; \mathbb{G}} \mathrm{v}(\mathfrak{t})-{ }^{c} \mathcal{D}_{a^{+}}^{q ; \mathbb{G}} \mathbf{v}(\mathfrak{t})\right|\right. \\
& +\left|{ }^{c} \mathcal{D}_{a^{+}}^{p ; \mathbb{G}}\left({ }^{c} \mathcal{D}_{a^{+}}^{q ; \mathbb{V}} \mathrm{v}(\mathfrak{t})\right)-{ }^{c} \mathcal{D}_{a^{+}}^{p ; \mathbb{G}}\left({ }^{c} \mathcal{D}_{a^{+}}^{q ; \mathbb{V}}(\mathfrak{t})\right)\right| \\
& \left.+\left|{ }^{c} \mathcal{D}_{a^{+}}^{r ; \mathbb{G}}\left({ }^{c} \mathcal{D}_{a^{+}}^{p ; \mathbb{G}}\left({ }^{c} \mathcal{D}_{a^{+}}^{q ; \mathbb{G}} \mathrm{v}(\mathfrak{t})\right)\right)-{ }^{c} \mathcal{D}_{a^{+}}^{r ; \mathbb{G}}\left({ }^{c} \mathcal{D}_{a^{+}}^{p ; \mathbb{G}}\left({ }^{c} \mathcal{D}_{a^{+}}^{q ; \mathbb{V}}(\mathfrak{t})\right)\right)\right|\right)
\end{aligned}
$$

for all $\mathrm{v}, \mathrm{v} \in \mathcal{C}$ with

$$
\begin{aligned}
& \chi^{*}\left(\left(\mathrm{v}(\mathfrak{t}),{ }^{c} \mathcal{D}_{a^{+}}^{q ; \mathbb{G}} \mathrm{v}(\mathfrak{t}),{ }^{c} \mathcal{D}_{a^{+}}^{p ; \mathbb{G}}\left({ }^{c} \mathcal{D}_{a^{+}}^{q ; \mathbb{G}} \mathrm{v}(\mathfrak{t})\right),\right.\right. \\
& \left.{ }^{c} \mathcal{D}_{a^{+}}^{r ; \mathbb{G}}\left({ }^{c} \mathcal{D}_{a^{+}}^{p ; \mathbb{G}}\left({ }^{c} \mathcal{D}_{a^{+}}^{q ; \mathbb{G}} \mathrm{v}(\mathfrak{t})\right)\right)\right), \\
& \left(\dot{\mathrm{v}}(\mathfrak{t}),{ }^{c} \mathcal{D}_{a^{+}}^{q ; \mathbb{G}} \dot{\mathrm{v}}(\mathfrak{t}),{ }^{c} \mathcal{D}_{a^{+}}^{p ; \mathbb{G}}\left({ }^{c} \mathcal{D}_{a^{+}}^{q ; \mathbb{G}} \dot{\mathrm{v}}(\mathfrak{t})\right),\right. \\
& \left.\left.{ }^{c} \mathcal{D}_{a^{+}}^{r ; \mathbb{G}}\left({ }^{c} \mathcal{D}_{a^{+}}^{p ; \mathbb{G}}\left({ }^{c} \mathcal{D}_{a^{+}}^{q ; \mathbb{V}}(\mathfrak{t})\right)\right)\right)\right) \geq 0
\end{aligned}
$$

for almost all $\mathfrak{t} \in[a, b]$. Thus there exists $\Upsilon \in \widehat{\mathfrak{H}}_{\mathrm{v}}$ such that

$$
\begin{aligned}
\left|\wp_{1}(\mathfrak{t})-\Upsilon\right| \leq & \frac{\phi(\mathfrak{t}) \mathcal{O}^{*}}{\|\phi\|} \psi\left(\left|\mathrm{v}_{1}-\mathrm{v}_{1}\right|+\left|{ }^{c} \mathcal{D}_{a^{+}}^{q ; \mathbb{G}} \mathrm{v}_{1}(\mathfrak{t})-{ }^{c} \mathcal{D}_{a^{+}}^{q ; \mathbb{G}} \mathrm{v}_{1}(\mathfrak{t})\right|\right. \\
& +\left|{ }^{c} \mathcal{D}_{a^{+}}^{p ; \mathbb{G}}\left({ }^{c} \mathcal{D}_{a^{+}}^{q ; \mathbb{G}} \mathrm{v}_{1}(\mathfrak{t})\right)-{ }^{c} \mathcal{D}_{a^{+}}^{p ; \mathbb{G}}\left({ }^{c} \mathcal{D}_{a^{+}}^{q ; \mathbb{G}} \boldsymbol{v}_{1}(\mathfrak{t})\right)\right|
\end{aligned}
$$




$$
\left.+\left|{ }^{c} \mathcal{D}_{a^{+}}^{r ; \mathbb{G}}\left({ }^{c} \mathcal{D}_{a^{+}}^{p ; \mathbb{G}}\left({ }^{c} \mathcal{D}_{a^{+}}^{q ; \mathbb{G}} \mathbf{V}_{1}(\mathfrak{t})\right)\right)-{ }^{c} \mathcal{D}_{a^{+}}^{r ; \mathbb{G}}\left({ }^{c} \mathcal{D}_{a^{+}}^{p ; \mathbb{G}}\left({ }^{c} \mathcal{D}_{a^{+}}^{q ; \mathbb{G}} \mathfrak{V}_{1}(\mathfrak{t})\right)\right)\right|\right)
$$

Now let $\mathfrak{N}^{*}:[0,1] \rightarrow \mathcal{P}(\mathcal{C})$ be a multivalued map defined as

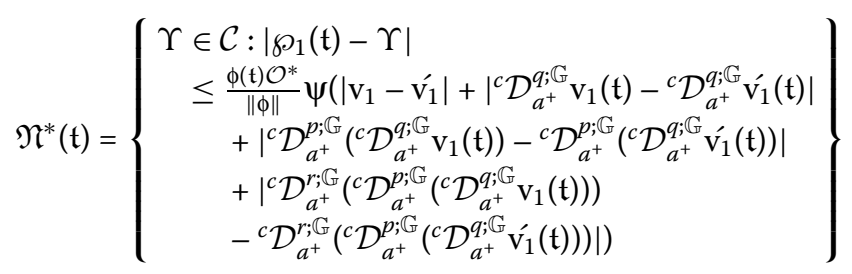

for all $\mathfrak{t} \in[a, b]$. As $\wp_{1}$ and

$$
\begin{aligned}
& \zeta=\frac{\phi(\mathfrak{t}) \mathcal{O}^{*}}{\|\phi\|} \psi\left(\left|\mathrm{v}_{1}-\mathfrak{v}_{1}\right|+\left|{ }^{c} \mathcal{D}_{a^{+}}^{q ; \mathrm{V}_{1}}(\mathfrak{t})-{ }^{c} \mathcal{D}_{a^{+}}^{q ; \mathbf{v}_{1}}(\mathfrak{t})\right|\right. \\
& +\left|{ }^{c} \mathcal{D}_{a^{+}}^{p ; \mathbb{G}}\left({ }^{c} \mathcal{D}_{a^{+}}^{q ; \mathbb{G}} \mathbf{V}_{1}(\mathfrak{t})\right)-{ }^{c} \mathcal{D}_{a^{+}}^{p, \mathbb{G}}\left({ }^{c} \mathcal{D}_{a^{+}}^{q ; \mathbb{G}} \dot{V}_{1}(\mathfrak{t})\right)\right| \\
& \left.+\left|{ }^{c} \mathcal{D}_{a^{+}}^{r ; \mathbb{G}}\left({ }^{c} \mathcal{D}_{a^{+}}^{p ; \mathbb{G}}\left({ }^{c} \mathcal{D}_{a^{+}}^{q ; \mathbb{G}} \mathrm{V}_{1}(\mathfrak{t})\right)\right)-{ }^{c} \mathcal{D}_{a^{+}}^{r ; \mathbb{G}}\left({ }^{c} \mathcal{D}_{a^{+}}^{p ; \mathbb{G}}\left({ }^{c} \mathcal{D}_{a^{+}}^{q ; \mathfrak{G}} \mathbf{V}_{1}(\mathfrak{t})\right)\right)\right|\right)
\end{aligned}
$$

are measurable, so is the multivalued function $\mathfrak{N}^{*}(\cdot) \cap \widehat{\mathfrak{H}}_{\mathrm{v}}(\cdot)$. Now let $\wp_{2} \in \widehat{\mathfrak{H}}_{\mathrm{v}}(\mathfrak{t})$ be such that

$$
\begin{aligned}
& \left|\wp_{1}(\mathfrak{t})-\wp_{2}(\mathfrak{t})\right| \leq \frac{\phi(\mathfrak{t}) \mathcal{O}^{*}}{\|\phi\|} \psi\left(\left|\mathrm{v}_{1}-\mathfrak{v}_{1}\right|+\left|{ }^{c} \mathcal{D}_{a^{+}}^{q ; \mathfrak{G}} \mathrm{v}_{1}(\mathfrak{t})-{ }^{c} \mathcal{D}_{a^{+}}^{q ; \mathbb{G}} \mathbf{v}_{1}(\mathfrak{t})\right|\right. \\
& +\left|{ }^{c} \mathcal{D}_{a^{+}}^{p ; \mathbb{G}}\left({ }^{c} \mathcal{D}_{a^{+}}^{q ; \mathfrak{G}} \mathrm{V}_{1}(\mathfrak{t})\right)-{ }^{c} \mathcal{D}_{a^{+}}^{p ; \mathbb{G}}\left({ }^{c} \mathcal{D}_{a^{+}}^{q ; \mathbb{G}} \mathbf{V}_{1}(\mathfrak{t})\right)\right| \\
& \left.+\left|{ }^{c} \mathcal{D}_{a^{+}}^{r ; \mathbb{G}}\left({ }^{c} \mathcal{D}_{a^{+}}^{p ; \mathbb{G}}\left({ }^{c} \mathcal{D}_{a^{+}}^{q ; \mathbb{G}} \mathrm{V}_{1}(\mathfrak{t})\right)\right)-{ }^{c} \mathcal{D}_{a^{+}}^{r ; \mathbb{G}}\left({ }^{c} \mathcal{D}_{a^{+}}^{p, \mathbb{G}}\left({ }^{c} \mathcal{D}_{a^{+}}^{q ; \mathbb{G}} \dot{V}_{1}(\mathfrak{t})\right)\right)\right|\right)
\end{aligned}
$$

for all $\mathfrak{t} \in[a, b]$. Let us define $\hbar_{2}^{*} \in \mathfrak{U}(\mathfrak{t})$ by

$$
\begin{aligned}
\hbar_{2}^{*}(\mathfrak{t})= & \mathrm{v}_{0}+\frac{\mathrm{v}_{1}(\mathbb{G}(\mathfrak{t})-\mathbb{G}(a))^{q}}{\Gamma(q+1)}+\frac{\mathrm{v}_{2}(\mathbb{G}(\mathfrak{t})-\mathbb{G}(a))^{q+p}}{\Gamma(q+p+1)} \\
& +\frac{\mathrm{v}_{3}(\mathbb{G}(\mathfrak{t})-\mathbb{G}(a))^{q+p+r}}{\Gamma(q+p+r+1)} \\
& +\int_{a}^{\mathfrak{t}} \mathbb{G}^{\prime}(\xi) \frac{(\mathbb{G}(\mathfrak{t})-\mathbb{G}(\xi))^{q+p+r+k-1}}{\Gamma(q+p+r+k)} \wp_{1}(\xi) \mathrm{d} \xi
\end{aligned}
$$

for all $\mathfrak{t} \in[a, b]$. Let $\sup _{\mathfrak{t} \in[a, b]}|\phi(\mathfrak{t})|=\|\phi\|$. Then

$$
\begin{aligned}
\left|\hbar_{1}^{*}(\mathfrak{t})-\hbar_{2}^{*}(\mathfrak{t})\right| & \leq \mathcal{I}_{a^{+}}^{q+p+r+k ; \mathbb{G}}\left|\widehat{\mathfrak{H}}_{\hbar_{1}^{*}}(\mathfrak{t})-\widehat{\mathfrak{H}}_{\hbar_{2}^{*}}(\mathfrak{t})\right| \\
& \leq \frac{(\mathbb{G}(b)-\mathbb{G}(a))^{q+p+r+k}}{\Gamma(q+p+r+k+1)}\|\phi(\mathfrak{t})\| \psi(\|\mathrm{v}-\dot{\mathrm{v}}\|) \frac{\mathcal{O}^{*}}{\|\phi(\mathfrak{t})\|} \\
& =\frac{(\mathbb{G}(b)-\mathbb{G}(a))^{q+p+r+k}}{\Gamma(q+p+r+k+1)} \mathcal{O}^{*} \psi(\|\mathrm{v}-\hat{\mathrm{v}}\|) .
\end{aligned}
$$

Also,

$$
\left|\left({ }^{c} \mathcal{D}_{a^{+}}^{q ; \mathbb{G}} \hbar_{1}^{*}\right)(\mathfrak{t})-\left({ }^{c} \mathcal{D}_{a^{+}}^{q ; \mathfrak{G}} \hbar_{2}^{*}\right)(\mathfrak{t})\right|
$$




$$
\begin{aligned}
& \leq \mathcal{I}_{a^{+}}^{p+r+k ; \mathbb{G}}\left|\widehat{\mathfrak{H}}_{\hbar_{1}^{*}}(\mathfrak{t})-\widehat{\mathfrak{H}}_{\hbar_{2}^{*}}(\mathfrak{t})\right| \\
& \leq \frac{(\mathbb{G}(b)-\mathbb{G}(a))^{p+r+k}}{\Gamma(p+r+k+1)}\|\phi(\mathfrak{t})\| \psi(\|\mathrm{v}-\dot{\mathrm{v}}\|) \frac{\mathcal{O}^{*}}{\|\phi(\mathfrak{t})\|} \\
&=\frac{(\mathbb{G}(b)-\mathbb{G}(a))^{p+r+k}}{\Gamma(p+r+k+1)} \mathcal{O}^{*} \psi(\|\mathrm{v}-\dot{\mathrm{v}}\|), \\
&\left|{ }^{c} \mathcal{D}_{a^{+}}^{p ; \mathbb{G}}\left({ }^{c} \mathcal{D}_{a^{+}}^{q ; \mathbb{G}} \hbar_{1}^{*}\right)(\mathfrak{t})-{ }^{c} \mathcal{D}_{a^{+}}^{p ; \mathbb{G}}\left({ }^{c} \mathcal{D}_{a^{+}}^{q ; \mathfrak{G}} \hbar_{2}^{*}\right)(\mathfrak{t})\right| \\
& \leq \leq \mathcal{I}_{a^{+}}^{r+k ; \mathbb{G}}\left|\widehat{\mathfrak{H}}_{\hbar_{1}^{*}}(\mathfrak{t})-\widehat{\mathfrak{H}}_{\hbar_{2}^{*}}(\mathfrak{t})\right| \\
& \leq \frac{(\mathbb{G}(b)-\mathbb{G}(a))^{r+k}}{\Gamma(r+k+1)}\|\phi(\mathfrak{t})\| \psi(\|\mathrm{v}-\dot{\mathrm{v}}\|) \frac{\mathcal{O}^{*}}{\|\phi(\mathfrak{t})\|} \\
& \quad=\frac{(\mathbb{G}(b)-\mathbb{G}(a))^{r+k}}{\Gamma(r+k+1)} \mathcal{O}^{*} \psi(\|\mathrm{v}-\dot{\mathrm{v}}\|),
\end{aligned}
$$

and

$$
\begin{aligned}
& \left|{ }^{c} \mathcal{D}_{a^{+}}^{r ; \mathbb{G}}\left({ }^{c} \mathcal{D}_{a^{+}}^{p ; \mathbb{G}}\left({ }^{c} \mathcal{D}_{a^{+}}^{q ; \mathfrak{G}} \hbar_{1}^{*}\right)\right)(\mathfrak{t})-{ }^{c} \mathcal{D}_{a^{+}}^{r ; \mathbb{G}}\left({ }^{c} \mathcal{D}_{a^{+}}^{p ; \mathbb{G}}\left({ }^{c} \mathcal{D}_{a^{+}}^{q ; \mathbb{G}} \hbar_{2}^{*}\right)\right)(\mathfrak{t})\right| \\
& \quad \leq \mathcal{I}_{a^{+}}^{k ; \mathbb{G}}\left|\widehat{\mathfrak{H}}_{\hbar_{1}^{*}}(\mathfrak{t})-\widehat{\mathfrak{H}}_{\hbar_{2}^{*}}(\mathfrak{t})\right| \\
& \quad \leq \frac{(\mathbb{G}(b)-\mathbb{G}(a))^{k}}{\Gamma(k+1)}\|\phi(\mathfrak{t})\| \psi(\|\mathrm{v}-\dot{\mathrm{v}}\|) \frac{\mathcal{O}^{*}}{\|\phi(\mathfrak{t})\|} \\
& \quad=\frac{(\mathbb{G}(b)-\mathbb{G}(a))^{k}}{\Gamma(k+1)} \mathcal{O}^{*} \psi(\|\mathrm{v}-\dot{\mathrm{v}}\|)
\end{aligned}
$$

for all $\mathfrak{t} \in[a, b]$. Hence

$$
\begin{aligned}
\left\|\hbar_{1}^{*}-\hbar_{2}^{*}\right\|= & \sup _{\mathfrak{t} \in[a, b]}\left|\hbar_{1}^{*}(\mathfrak{t})-\hbar_{2}^{*}(\mathfrak{t})\right|+\sup _{\mathfrak{t} \in[a, b]}\left|{ }^{c} \mathcal{D}_{a^{+}}^{q ; \mathfrak{G}} \hbar_{1}^{*}(\mathfrak{t})-\hbar_{2}^{*}(\mathfrak{t})\right| \\
& +\sup _{\mathfrak{t} \in[a, b]}\left|{ }^{c} \mathcal{D}_{a^{+}}^{p ; \mathbb{G}}\left({ }^{c} \mathcal{D}_{a^{+}}^{q ; \mathbb{G}} \hbar_{1}^{*}(\mathfrak{t})\right)-{ }^{c} \mathcal{D}_{a^{+}}^{p ; \mathbb{G}}\left({ }^{c} \mathcal{D}_{a^{+}}^{q ; \mathbb{G}} \hbar_{2}^{*}(\mathfrak{t})\right)\right| \\
& +\sup _{\mathfrak{t} \in[a, b]} \mid{ }^{c} \mathcal{D}_{a^{+}}^{r ; \mathbb{G}}\left({ }^{c} \mathcal{D}_{a^{+}}^{p ; \mathbb{G}}\left({ }^{c} \mathcal{D}_{a^{+}}^{q ; \mathbb{G}} \hbar_{1}^{*}(\mathfrak{t})\right)\right) \\
& -{ }^{c} \mathcal{D}_{a^{+}}^{r ; \mathbb{G}}\left({ }^{c} \mathcal{D}_{a^{+}}^{p ; \mathbb{G}}\left({ }^{c} \mathcal{D}_{a^{+}}^{q ; \mathbb{G}} \hbar_{2}^{*}(\mathfrak{t})\right)\right) \mid \\
\leq & {\left[\frac{(\mathbb{G}(b)-\mathbb{G}(a)))^{q+p+r+k}}{\Gamma(q+p+r+k+1)}+\frac{(\mathbb{G}(b)-\mathbb{G}(a))^{p+r+k}}{\Gamma(p+r+k+1)}\right.} \\
& \left.+\frac{(\mathbb{G}(b)-\mathbb{G}(a))^{r+k}}{\Gamma(r+k+1)}+\frac{(\mathbb{G}(b)-\mathbb{G}(a))^{k}}{\Gamma(k+1)}\right] \mathcal{O}^{*} \psi(\|\mathrm{v}-\hat{v}\|) \\
= & \psi(\|\mathrm{v}-\hat{v}\|),
\end{aligned}
$$

and thus

$$
\alpha(\mathrm{v}, \hat{\mathrm{v}}) \mathcal{H}_{d}(\mathfrak{U}(\mathrm{v}), \mathfrak{U}(\hat{\mathrm{v}})) \leq \psi(\|\mathrm{v}-\dot{\mathrm{v}}\|)
$$

for all $\mathrm{v}, \mathrm{v} \in \mathcal{C}$, which implies that $\mathfrak{U}$ is an $\alpha-\psi$-contraction. Now, let $\mathrm{v} \in \mathcal{C}$ and $\mathbf{v} \in \mathfrak{U}(\mathrm{v})$ be two functions such that $\alpha(\mathrm{v}, \hat{\mathrm{v}}) \geq 1$. In this case,

$$
\chi^{*}\left(\left(\mathrm{v}(\mathfrak{t}),{ }^{c} \mathcal{D}_{a^{+}}^{q ; \mathbb{G}} \mathrm{v}(\mathfrak{t}),{ }^{c} \mathcal{D}_{a^{+}}^{p ; \mathbb{G}}\left({ }^{c} \mathcal{D}_{a^{+}}^{q ; \mathfrak{G}} \mathrm{v}(\mathfrak{t})\right),{ }^{c} \mathcal{D}_{a^{+}}^{r ; \mathbb{G}}\left({ }^{c} \mathcal{D}_{a^{+}}^{p ; \mathbb{G}}\left({ }^{c} \mathcal{D}_{a^{+}}^{q ; \mathfrak{G}} \mathrm{v}(\mathfrak{t})\right)\right)\right),\right.
$$




$$
\left.\left(\dot{\mathrm{v}}(\mathfrak{t}),{ }^{c} \mathcal{D}_{a^{+}}^{q ; \mathfrak{G}}(\mathfrak{t}),{ }^{c} \mathcal{D}_{a^{+}}^{p ; \mathbb{G}}\left({ }^{c} \mathcal{D}_{a^{+}}^{q ; \mathfrak{G}} \dot{\mathrm{V}}(\mathfrak{t})\right),{ }^{c} \mathcal{D}_{a^{+}}^{r ; \mathbb{G}}\left({ }^{c} \mathcal{D}_{a^{+}}^{p ; \mathbb{G}}\left({ }^{c} \mathcal{D}_{a^{+}}^{q ; \mathfrak{G}}(\mathfrak{t})\right)\right)\right)\right) \geq 0
$$

so there exists $\Upsilon \in \mathfrak{U}(\hat{v})$ such that

$$
\begin{gathered}
\chi^{*}\left(\left(\hat{\mathrm{v}}(\mathfrak{t}),{ }^{c} \mathcal{D}_{a^{+}}^{q ; \mathbb{G}} \mathbf{v}(\mathfrak{t}),{ }^{c} \mathcal{D}_{a^{+}}^{p ; \mathbb{G}}\left({ }^{c} \mathcal{D}_{a^{+}}^{q ; \mathbb{G}} \dot{\mathrm{v}}(\mathfrak{t})\right),\right.\right. \\
\left.{ }^{c} \mathcal{D}_{a^{+}}^{r ; \mathbb{G}}\left({ }^{c} \mathcal{D}_{a^{+}}^{p ; \mathbb{G}}\left({ }^{c} \mathcal{D}_{a^{+}}^{q ; \mathbb{G}} \dot{\mathrm{v}}(\mathfrak{t})\right)\right)\right), \\
\left(\Upsilon(\mathfrak{t}),{ }^{c} \mathcal{D}_{a^{+}}^{q ; \mathbb{G}} \Upsilon(\mathfrak{t}),{ }^{c} \mathcal{D}_{a^{+}}^{p ; \mathbb{G}}\left({ }^{c} \mathcal{D}_{a^{+}}^{q ; \mathbb{G}} \Upsilon(\mathfrak{t})\right),\right. \\
\left.\left.{ }^{c} \mathcal{D}_{a^{+}}^{r \cdot \mathbb{G}}\left({ }^{c} \mathcal{D}_{a^{+}}^{p ; \mathbb{G}}\left({ }^{c} \mathcal{D}_{a^{+}}^{q ; \mathbb{G}} \Upsilon(\mathfrak{t})\right)\right)\right)\right) \geq 0 .
\end{gathered}
$$

From this it follows that $\alpha\left(v^{\prime}, \Upsilon\right) \geq 1$, which means that the operator $\mathfrak{U}$ is an $\alpha$-admissible. Now suppose that $\mathrm{v}_{0} \in \mathcal{C}$ and $\mathfrak{v} \in \mathfrak{U}\left(\mathrm{v}_{0}\right)$ are such that

$$
\begin{gathered}
\chi^{*}\left(\left(\mathrm{v}_{0}(\mathfrak{t}),{ }^{c} \mathcal{D}_{a^{+}}^{q ; \mathbb{G}} \mathrm{v}_{0}(\mathfrak{t}),{ }^{c} \mathcal{D}_{a^{+}}^{p ; \mathbb{G}}\left({ }^{c} \mathcal{D}_{a^{+}}^{q ; \mathbb{G}} \mathrm{v}_{0}(\mathfrak{t})\right),\right.\right. \\
\left.{ }^{c} \mathcal{D}_{a^{+}}^{r ; \mathbb{G}}\left({ }^{c} \mathcal{D}_{a^{+}}^{p ; \mathbb{G}}\left({ }^{c} \mathcal{D}_{a^{+}}^{q ; \mathbb{G}} \mathrm{v}_{0}(\mathfrak{t})\right)\right)\right), \\
\left(\hat{\mathrm{v}}(\mathfrak{t}),{ }^{c} \mathcal{D}_{a^{+}}^{q ; \mathbb{V}} \hat{\mathrm{v}}(\mathfrak{t}),{ }^{c} \mathcal{D}_{a^{+}}^{p ; \mathbb{G}}\left({ }^{c} \mathcal{D}_{a^{+}}^{q ; \mathbb{V}} \hat{\mathrm{v}}(\mathfrak{t})\right),\right. \\
\left.\left.{ }^{c} \mathcal{D}_{a^{+}}^{r, \mathbb{G}}\left({ }^{c} \mathcal{D}_{a^{+}}^{p ; \mathbb{G}}\left({ }^{c} \mathcal{D}_{a^{+}}^{q ; \mathbb{V}} \dot{\mathrm{v}}(\mathfrak{t})\right)\right)\right)\right) \geq 0
\end{gathered}
$$

for all $\mathfrak{t} \in[a, b]$. Subsequently, we have $\alpha\left(\mathrm{v}_{0}, \mathrm{v}\right) \geq 1$. Consider $\left\{\mathrm{v}_{n}\right\} \subseteq \mathcal{C}$ such that $\mathrm{v}_{n} \rightarrow \mathrm{v}$ and $\alpha\left(\mathrm{v}_{n}, \mathrm{v}_{n+1}\right) \geq 1$. Then we get

$$
\begin{aligned}
& \chi^{*}\left(\left(\mathrm{v}_{n}(\mathfrak{t}),{ }^{c} \mathcal{D}_{a^{+}}^{q ; \mathbb{G}} \mathrm{v}_{n}(\mathfrak{t}),{ }^{c} \mathcal{D}_{a^{+}}^{p ; \mathbb{G}}\left({ }^{c} \mathcal{D}_{a^{+}}^{q ; \mathbb{G}} \mathrm{v}_{n}(\mathfrak{t})\right),\right.\right. \\
& \left.{ }^{c} \mathcal{D}_{a^{+}}^{r ; \mathbb{G}}\left({ }^{c} \mathcal{D}_{a^{+}}^{p ; \mathbb{G}}\left({ }^{c} \mathcal{D}_{a^{+}}^{q ; \mathbb{G}} \mathrm{v}_{n}(\mathfrak{t})\right)\right)\right), \\
& \left(\mathrm{v}_{n+1}(\mathfrak{t}),{ }^{c} \mathcal{D}_{a^{+}}^{q ; \mathbb{G}} \mathbf{v}_{n+1}(\mathfrak{t}),{ }^{c} \mathcal{D}_{a^{+}}^{p ; \mathbb{G}}\left({ }^{c} \mathcal{D}_{a^{+}}^{q ; \mathbb{G}} \mathbf{v}_{n+1}(\mathfrak{t})\right),\right. \\
& \left.\left.{ }^{c} \mathcal{D}_{a^{+}}^{r ; \mathbb{G}}\left({ }^{c} \mathcal{D}_{a^{+}}^{p ; \mathbb{G}}\left({ }^{c} \mathcal{D}_{a^{+}}^{q ; \mathbb{G}} \mathbf{v}_{n+1}(\mathfrak{t})\right)\right)\right)\right) \geq 0 .
\end{aligned}
$$

By hypothesis (C8) there is a subsequence $\left\{\mathrm{v}_{n_{j}}\right\}$ of $\left\{\mathrm{v}_{n}\right\}$ such that

$$
\begin{aligned}
& \chi^{*}\left(\left(\mathrm{v}_{n_{j}}(\mathfrak{t}),{ }^{c} \mathcal{D}_{a^{+}}^{q ; \mathfrak{G}} \mathrm{v}_{n_{j}}(\mathfrak{t}),{ }^{c} \mathcal{D}_{a^{+}}^{p ; \mathbb{G}}\left({ }^{c} \mathcal{D}_{a^{+}}^{q ; \mathfrak{G}} \mathrm{v}_{n_{j}}(\mathfrak{t})\right),\right.\right. \\
& \left.{ }^{c} \mathcal{D}_{a^{+}}^{r ; \mathbb{G}}\left({ }^{c} \mathcal{D}_{a^{+}}^{p ; \mathbb{G}}\left({ }^{c} \mathcal{D}_{a^{+}}^{q ; \mathfrak{G}} \mathbf{V}_{n_{j}}(\mathfrak{t})\right)\right)\right), \\
& \left(\mathrm{v}(\mathfrak{t}),{ }^{c} \mathcal{D}_{a^{+}}^{q ; \mathbb{G}} \mathrm{v}(\mathfrak{t}),{ }^{c} \mathcal{D}_{a^{+}}^{p ; \mathbb{G}}\left({ }^{c} \mathcal{D}_{a^{+}}^{q ; \mathbb{G}} \mathrm{v}(\mathfrak{t})\right),\right. \\
& \left.\left.{ }^{c} \mathcal{D}_{a^{+}}^{r ; \mathbb{G}}\left({ }^{c} \mathcal{D}_{a^{+}}^{p ; \mathbb{G}}\left({ }^{c} \mathcal{D}_{a^{+}}^{q ; \mathbb{G}} \mathrm{v}(\mathfrak{t})\right)\right)\right)\right) \geq 0 .
\end{aligned}
$$

Thus $\alpha\left(\mathrm{v}_{n_{j}}, \mathrm{v}\right) \geq 1(\forall j)$, that is, $\mathcal{C}$ has the property $C_{\alpha}$. Theorem 2.12 guarantees that $\mathfrak{N}$ has a fixed point, which is the solution of the inclusion BVP (46).

Theorem 5.2 Consider a multifunction $\mathfrak{H}:[a, b] \times \mathcal{C} \times \mathcal{C} \rightarrow \mathcal{P}(\mathcal{C})$. Assume that:

(C11) $\psi: \mathbb{R}_{\geq 0} \rightarrow \mathbb{R}_{\geq 0}$ is u.s.c nondecreasing map with $\liminf _{\mathrm{v} \rightarrow \infty}(\mathrm{v}-\psi(\mathrm{v}))>0$ and $\psi(\mathrm{v})<\mathrm{v}$ for all $\mathrm{v}>0$;

(C12) The operator $\mathfrak{H}:[a, b] \times \mathcal{C} \times \mathcal{C} \rightarrow \mathcal{P}_{\mathrm{CP}}(\mathcal{C})$ is integrable and bounded, and $\mathfrak{H}\left(\cdot, \mathrm{v}_{1}^{\prime}, \mathrm{v}_{2}^{\prime}, \mathrm{v}_{3}^{\prime}, \mathrm{v}_{4}^{\prime}\right):[a, b] \rightarrow \mathcal{P}_{\mathrm{CP}}(\mathcal{C})$ is measurable for all $\mathrm{v}_{1}, \mathrm{v}_{2}, \mathrm{v}_{3}, \mathrm{v}_{4} \in \mathcal{C} ;$ 
(C13) There is $\phi \in C([a, b],[0, \infty))$ such that

$$
\mathcal{H}_{d}\left(\mathfrak{H}\left(\mathfrak{t}, \mathrm{v}_{1}, \mathrm{v}_{2}, \mathrm{v}_{3}, \mathrm{v}_{4}\right), \mathfrak{H}\left(\mathfrak{t}, \mathbf{v}_{1}^{\prime}, \dot{v}_{2}, \mathbf{v}_{3}^{\prime}, \mathbf{v}_{4}^{\prime}\right)\right) \leq \phi(\mathfrak{t}) \mathcal{O}^{*} \psi\left(\sum_{k=1}^{4}\left|\mathrm{v}_{k}-\dot{v}_{k}\right|\right)
$$

for all $\mathbf{v}_{k}, \dot{\mathbf{v}}_{k} \in \mathcal{C}(k=1,2,3,4)$, where $\mathcal{O}^{*}=\mathcal{O}^{-1}$;

(xv) $\mathfrak{U}$ has the (AEP)-property.

Then the inclusion BVP (46) has a solution.

Proof We have to prove that $\mathfrak{U}: \mathcal{C} \rightarrow \mathcal{P}(\mathcal{C})$ includes end points. Firstly, we must prove that $\mathfrak{U}(\mathrm{v})$ is closed for every $\mathrm{v} \in \mathcal{C}$. Since the mapping

$$
\mathfrak{t} \rightarrow \mathfrak{H}\left(\mathfrak{t}, \mathrm{v}(\mathfrak{t}),{ }^{c} \mathcal{D}_{a^{+}}^{q ; \mathbb{G}} \mathrm{v}(\mathfrak{t}),{ }^{c} \mathcal{D}_{a^{+}}^{p ; \mathbb{G}}\left({ }^{c} \mathcal{D}_{a^{+}}^{q ; \mathfrak{G}} \mathrm{v}(\mathfrak{t})\right),{ }^{c} \mathcal{D}_{a^{+}}^{r ; \mathbb{G}}\left({ }^{c} \mathcal{D}_{a^{+}}^{p ; \mathbb{G}}\left({ }^{c} \mathcal{D}_{a^{+}}^{q ; \mathfrak{G}} \mathrm{v}(\mathfrak{t})\right)\right)\right)
$$

is closed-valued and measurable for $\mathrm{v} \in \mathcal{C}$, it has a measurable selection, and $\mathfrak{S}_{\mathfrak{H}, \mathfrak{v}}^{*} \neq \emptyset$. By applying the same deduction as in the proof of Theorem 5.1, we may simply verify that $\mathfrak{U}(\mathrm{v})$ is closed. Also, $\mathfrak{U}(\mathrm{v})$ is bounded because of the compactness of $\mathfrak{H}$. Finally, it is simple to prove that

$$
\mathcal{H}_{d}(\mathfrak{U}(\mathrm{v}), \mathfrak{U}(\Upsilon)) \leq \psi(\|\mathrm{v}-\Upsilon\|)
$$

Suppose that $\mathrm{v}, \Upsilon \in \mathcal{C}$ and $\hbar_{1}^{*} \in \mathfrak{U}(\Upsilon)$. Choose $\wp_{1} \in \mathfrak{S}_{\mathfrak{H}, \Upsilon}$ such that

$$
\begin{aligned}
\hbar_{1}^{*}(\mathfrak{t})= & \mathrm{v}_{0}+\frac{\mathrm{v}_{1}(\mathbb{G}(\mathfrak{t})-\mathbb{G}(a))^{q}}{\Gamma(q+1)}+\frac{\mathrm{v}_{2}(\mathbb{G}(\mathfrak{t})-\mathbb{G}(a))^{q+p}}{\Gamma(q+p+1)} \\
& +\frac{\mathrm{v}_{3}(\mathbb{G}(\mathfrak{t})-\mathbb{G}(a))^{q+p+r}}{\Gamma(q+p+r+1)} \\
& +\int_{a}^{\mathfrak{t}} \mathbb{G}^{\prime}(\xi) \frac{(\mathbb{G}(\mathfrak{t})-\mathbb{G}(\xi))^{q+p+r+k-1}}{\Gamma(q+p+r+k)} \wp_{1}(\xi) \mathrm{d} \xi
\end{aligned}
$$

for all $\mathfrak{t} \in[a, b]$. As

$$
\begin{aligned}
\mathcal{H}_{d}\left(\widehat{\mathfrak{H}}_{\mathrm{v}}(\mathfrak{t}), \widehat{\mathfrak{H}}_{\Upsilon}(\mathfrak{t})\right) \leq & \phi(\mathfrak{t}) \mathcal{O}^{*} \psi\left(|\mathrm{v}-\Upsilon|+\left|{ }^{c} \mathcal{D}_{a^{+}}^{q ; \mathfrak{G}} \mathrm{v}(\mathfrak{t})-{ }^{c} \mathcal{D}_{a^{+}}^{q ; \mathfrak{G}} \Upsilon(\mathfrak{t})\right|\right. \\
& +\left|{ }^{c} \mathcal{D}_{a^{+}}^{p ; \mathbb{G}}\left({ }^{c} \mathcal{D}_{a^{+}}^{q ; \mathfrak{G}} \mathrm{v}(\mathfrak{t})\right)-{ }^{c} \mathcal{D}_{a^{+}}^{p ; \mathbb{G}}\left({ }^{c} \mathcal{D}_{a^{+}}^{q ; \mathbb{G}} \Upsilon(\mathfrak{t})\right)\right| \\
& +\mid{ }^{c} \mathcal{D}_{a^{+}}^{r, \mathbb{G}}\left({ }^{c} \mathcal{D}_{a^{+}}^{p ; \mathbb{G}}\left({ }^{c} \mathcal{D}_{a^{+}}^{q ; \mathfrak{G}} \mathrm{v}(\mathfrak{t})\right)\right) \\
& \left.-{ }^{c} \mathcal{D}_{a^{+}}^{r, \mathbb{G}}\left({ }^{c} \mathcal{D}_{a^{+}}^{p ; \mathbb{G}}\left({ }^{c} \mathcal{D}_{a^{+}}^{q ; \mathbb{G}} \Upsilon(\mathfrak{t})\right)\right) \mid\right)
\end{aligned}
$$

for all $\mathfrak{t} \in[a, b]$, there exists $\phi^{*} \in \widehat{\mathfrak{H}}_{\mathrm{v}}(\mathfrak{t})$ such that

$$
\begin{aligned}
\left|\wp_{1}(\mathfrak{t})-\phi^{*}\right| \leq & \phi(\mathfrak{t}) \mathcal{O}^{*} \psi\left(|\mathrm{v}(\mathfrak{t})-\Upsilon(\mathfrak{t})|+\left|{ }^{\mathcal{C}} \mathfrak{D}_{0}^{1} \mathrm{v}(\mathfrak{t})-{ }^{\mathcal{C}} \mathfrak{D}_{0}^{1} \Upsilon(\mathfrak{t})\right|\right. \\
& +\left|{ }^{c} \mathcal{D}_{a^{+}}^{p ; \mathbb{G}}\left({ }^{c} \mathcal{D}_{a^{+}}^{q ; \mathbb{G}} \mathrm{v}(\mathfrak{t})\right)-{ }^{c} \mathcal{D}_{a^{+}}^{p ; \mathbb{G}}\left({ }^{c} \mathcal{D}_{a^{+}}^{q ; \mathbb{G}} \Upsilon(\mathfrak{t})\right)\right| \\
& +\mid{ }^{c} \mathcal{D}_{a^{+}}^{r ; \mathbb{G}}\left({ }^{c} \mathcal{D}_{a^{+}}^{p ; \mathbb{G}}\left({ }^{c} \mathcal{D}_{a^{+}}^{q ; \mathbb{G}} \mathrm{v}(\mathfrak{t})\right)\right) \\
& \left.-{ }^{c} \mathcal{D}_{a^{+}}^{r ; \mathbb{G}}\left({ }^{c} \mathcal{D}^{p ; \mathbb{G}}\left({ }^{c} \mathcal{D}_{a^{+}}^{q ; \mathbb{G}} \Upsilon(\mathfrak{t})\right)\right) \mid\right)
\end{aligned}
$$


for all $\mathfrak{t} \in[a, b]$. Consider the multivalued map $\mathfrak{O}^{*}:[a, b] \rightarrow \mathcal{P}(\mathcal{C})$ defined by

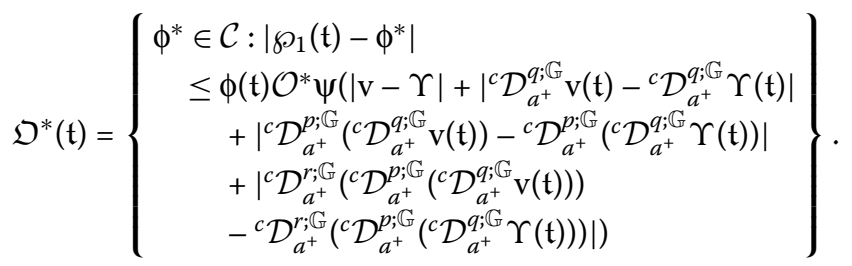

By the measurability of $\wp_{1}$ and

$$
\begin{aligned}
\phi^{*}= & \phi(\mathfrak{t}) \mathcal{O}^{*} \psi\left(|\mathrm{v}-\Upsilon|+\left|{ }^{c} \mathcal{D}_{a^{+}}^{q ; \mathbb{G}} \mathrm{v}(\mathfrak{t})-{ }^{c} \mathcal{D}_{a^{+}}^{q ; \mathbb{G}} \Upsilon(\mathfrak{t})\right|\right. \\
& +\left|{ }^{c} \mathcal{D}_{a^{+}}^{p ; \mathbb{G}}\left({ }^{c} \mathcal{D}_{a^{+}}^{q ; \mathbb{G}} \mathrm{v}(\mathfrak{t})\right)-{ }^{c} \mathcal{D}_{a^{+}}^{p ; \mathbb{G}}\left({ }^{c} \mathcal{D}_{a^{+}}^{q ; \mathbb{G}} \Upsilon(\mathfrak{t})\right)\right| \\
& \left.+\left|{ }^{c} \mathcal{D}_{a^{+}}^{r \mathbb{G}}\left({ }^{c} \mathcal{D}_{a^{+}}^{p ; \mathbb{G}}\left({ }^{c} \mathcal{D}_{a^{+}}^{q ; \mathbb{G}} \mathrm{v}(\mathfrak{t})\right)\right)-{ }^{c} \mathcal{D}_{a^{+}}^{r, \mathbb{G}}\left({ }^{c} \mathcal{D}_{a^{+}}^{p ; \mathbb{G}}\left({ }^{c} \mathcal{D}_{a^{+}}^{q ; \mathbb{G}} \Upsilon(\mathfrak{t})\right)\right)\right|\right)
\end{aligned}
$$

it is obvious that that multifunction $\mathfrak{O}^{*}(\cdot) \cap \widehat{\mathfrak{H}}_{\mathrm{v}}(\cdot)$ is also measurable. Now we take $\wp_{2} \in$ $\widehat{\mathfrak{H}}_{\mathrm{v}}(\mathfrak{t})$ such that

$$
\begin{aligned}
\left|\wp_{1}(\mathfrak{t})-\wp_{2}(\mathfrak{t})\right| \leq & \phi(\mathfrak{t}) \mathcal{O}^{*} \psi\left(|\mathrm{v}-\Upsilon|+\left|{ }^{c} \mathcal{D}_{a^{+}}^{q ; \mathbb{G}} \mathrm{v}(\mathfrak{t})-{ }^{c} \mathcal{D}_{a^{+}}^{q ; \mathfrak{G}} \Upsilon(\mathfrak{t})\right|\right. \\
& +\left|{ }^{c} \mathcal{D}_{a^{+}}^{p ; \mathbb{G}}\left({ }^{c} \mathcal{D}_{a^{+}}^{q ; \mathfrak{G}} \mathrm{v}(\mathfrak{t})\right)-{ }^{c} \mathcal{D}_{a^{+}}^{p ; \mathbb{G}}\left({ }^{c} \mathcal{D}_{a^{+}}^{q ; \mathbb{G}} \Upsilon(\mathfrak{t})\right)\right| \\
& \left.+\left|{ }^{c} \mathcal{D}_{a^{+}}^{r ; \mathbb{G}}\left({ }^{c} \mathcal{D}_{a^{+}}^{p ; \mathbb{G}}\left({ }^{c} \mathcal{D}_{a^{+}}^{q ; \mathbb{G}} \mathrm{v}(\mathfrak{t})\right)\right)-{ }^{c} \mathcal{D}_{a^{+}}^{r ; \mathbb{G}}\left({ }^{c} \mathcal{D}_{a^{+}}^{p ; \mathbb{G}}\left({ }^{c} \mathcal{D}_{a^{+}}^{q ; \mathbb{G}} \Upsilon(\mathfrak{t})\right)\right)\right|\right)
\end{aligned}
$$

for all $\mathfrak{t} \in[a, b]$. Choose $\hbar_{2}^{*} \in \mathfrak{U}(\mathrm{v})$ such that

$$
\begin{aligned}
\hbar_{2}^{*}(\mathfrak{t})= & \mathrm{v}_{0}+\frac{\mathrm{v}_{1}(\mathbb{G}(\mathfrak{t})-\mathbb{G}(a))^{q}}{\Gamma(q+1)}+\frac{\mathrm{v}_{2}(\mathbb{G}(\mathfrak{t})-\mathbb{G}(a))^{q+p}}{\Gamma(q+p+1)} \\
& +\frac{\mathrm{v}_{3}(\mathbb{G}(\mathfrak{t})-\mathbb{G}(a))^{q+p+r}}{\Gamma(q+p+r+1)} \\
& +\int_{a}^{\mathfrak{t}} \mathbb{G}^{\prime}(\xi) \frac{(\mathbb{G}(\mathfrak{t})-\mathbb{G}(\xi))^{q+p+r+k-1}}{\Gamma(q+p+r+k)} \wp_{2}(\xi) \mathrm{d} \xi
\end{aligned}
$$

for all $\mathfrak{t} \in[a, b]$. By the same argument as in Theorem 5.1 we get

$$
\begin{aligned}
\left\|\hbar_{1}^{*}-\hbar_{2}^{*}\right\|= & \sup _{\mathfrak{t} \in[a, b]}\left|\hbar_{1}^{*}(\mathfrak{t})-\hbar_{2}^{*}(\mathfrak{t})\right|+\sup _{\mathfrak{t} \in[a, b]}\left|{ }^{c} \mathcal{D}_{a^{+}}^{q ; \mathfrak{G}} \hbar_{1}^{*}(\mathfrak{t})-\hbar_{2}^{*}(\mathfrak{t})\right| \\
& +\sup _{\mathfrak{t} \in[a, b]}\left|{ }^{c} \mathcal{D}_{a^{+}}^{p ; \mathbb{G}}\left({ }^{c} \mathcal{D}_{a^{+}}^{q ; \mathbb{G}} \hbar_{1}^{*}(\mathfrak{t})\right)-{ }^{c} \mathcal{D}_{a^{+}}^{p ; \mathbb{G}}\left({ }^{c} \mathcal{D}_{a^{+}}^{q ; \mathbb{G}} \hbar_{2}^{*}(\mathfrak{t})\right)\right| \\
& +\sup _{\mathfrak{t} \in[a, b]} \mid{ }^{c} \mathcal{D}_{a^{+}}^{r ; \mathbb{G}}\left({ }^{c} \mathcal{D}_{a^{+}}^{p ; \mathbb{G}}\left({ }^{c} \mathcal{D}_{a^{+}}^{q ; \mathbb{G}} \hbar_{1}^{*}(\mathfrak{t})\right)\right) \\
& -{ }^{c} \mathcal{D}_{a^{+}}^{r ; \mathbb{G}}\left({ }^{c} \mathcal{D}_{a^{+}}^{p: \mathbb{G}}\left({ }^{c} \mathcal{D}_{a^{+}}^{q ; \mathbb{G}} \hbar_{2}^{*}(\mathfrak{t})\right)\right) \mid \\
\leq & {\left[\frac{(\mathbb{G}(b)-\mathbb{G}(a))^{q+p+r+k}}{\Gamma(q+p+r+k+1)}+\frac{(\mathbb{G}(b)-\mathbb{G}(a))^{p+r+k}}{\Gamma(p+r+k+1)}\right.} \\
& \left.+\frac{(\mathbb{G}(b)-\mathbb{G}(a))^{r+k}}{\Gamma(r+k+1)}+\frac{(\mathbb{G}(b)-\mathbb{G}(a))^{k}}{\Gamma(k+1)}\right] \mathcal{O}^{*} \psi(\|\mathrm{v}-\dot{\mathrm{v}}\|) \\
= & \psi(\|\mathrm{v}-\hat{\mathrm{v}}\|) .
\end{aligned}
$$


Hence

$$
\mathcal{H}_{d}(\mathfrak{U}(\mathrm{v}), \mathfrak{U}(\Upsilon)) \leq \psi(\|\mathrm{v}-\Upsilon\|)
$$

for all $\mathrm{v}, \Upsilon \in \mathcal{C}$. By using hypothesis (xv) we can easily find that $\mathfrak{U}$ has the (AEP)-property. By Theorem 2.13 there exists $\mathrm{v}^{*} \in \mathcal{C}$ such that $\mathfrak{U}\left(\mathrm{v}^{*}\right)=\left\{\mathrm{v}^{*}\right\}$. This implies that $\mathrm{v}^{*}$ satisfies the given problem (46), and the proof is completed.

\section{Numerical applications}

Here we give some examples of fractional $\mathbb{G}$-snap systems based on numerical simulations to analyze their solutions. In these examples, we consider different cases of the function $\mathbb{G}$ to cover the Caputo, Caputo-Hadamard, and Katugampola versions. For numerical computations, one can use Algorithms 1, 2 and 3.

Example 6.1 Based on system (4), we consider the nonlinear fractional $\psi$-snap BVP

$$
\left\{\begin{array}{l}
{ }^{c} \mathcal{D}_{1.1+}^{0.34 ; \mathfrak{G}} \mathrm{v}(\mathfrak{t})=\mathrm{u}(\mathfrak{t}), \quad 1.1 \leq \mathfrak{t} \leq 2.6, \mathrm{v}(1.1)=2.25, \\
{ }^{c} \mathcal{D}_{1.1^{+}}^{0.86 ; \mathfrak{G}} \mathrm{u}(\mathfrak{t})=\mathrm{w}(\mathfrak{t}), \quad \mathrm{u}(1.1)=-1.69, \\
{ }^{c} \mathcal{D}_{1.1^{+}}^{0.54 ; \mathfrak{G}} \mathrm{w}(\mathfrak{t})=\mathrm{x}(\mathfrak{t}), \quad \mathrm{w}(1.1)=3.12, \\
{ }^{c} \mathcal{D}_{1.1^{+}}^{0.25 ; \mathfrak{G}} \mathrm{x}(\mathfrak{t})=h(\mathfrak{t}, \mathrm{v}, \mathrm{u}, \mathrm{w}, \mathrm{x}), \quad \mathrm{x}(1.1)=-4.71,
\end{array}\right.
$$

where

$$
\begin{aligned}
h(\mathrm{t}, \mathrm{v}, \mathrm{u}, \mathrm{w}, \mathrm{x})= & \frac{\sqrt{\mathfrak{t}}}{12(1+\sqrt{\mathfrak{t}})}+\frac{|\mathrm{v}(\mathfrak{t})|}{30(1+\exp (\mid \mathrm{v}(\mathfrak{t})) \mid)}+\frac{1}{15} \tan ^{-1}(\mathrm{u}(\mathfrak{t})) \\
& +\frac{\mathfrak{t}}{40} \frac{\sin ^{2}(\mathrm{w}(\mathfrak{t}))}{5+\sin ^{2}(\mathrm{w}(\mathfrak{t}))}+\frac{3 \mathfrak{t}}{20} \frac{\left|\sin ^{-1}(\mathrm{x}(\mathfrak{t}))\right|}{8+\left|\sin ^{-1}(\mathrm{x}(\mathfrak{t}))\right|}
\end{aligned}
$$

for $\mathfrak{t} \in[1.1,2.6]$. It is clear that $a=1.1, b=2.6, q=0.34 \in(0,1], \mathrm{v}(0)=\mathrm{v}_{0}=2.25, p=0.86 \in$ $(0,1], \mathrm{u}(0)=\mathrm{v}_{1}=-1.69, r=0.54 \in(0,1], \mathrm{w}(0)=\mathrm{v}_{2}=3.12, k=0.25 \in(0,1], \mathrm{x}(0)=\mathrm{v}_{3}=$ -4.71 , and

$$
\begin{aligned}
h\left(\mathfrak{t}_{1} \mathrm{v}_{1}, \mathrm{v}_{2}, \mathrm{v}_{3}, \mathrm{v}_{3}\right)= & \frac{\sqrt{\mathfrak{t}}}{12(1+\sqrt{\mathfrak{t}})}+\frac{\left|\mathrm{v}_{1}\right|}{30\left(1+\exp \left(\mid \mathrm{v}_{1}\right) \mid\right)}+\frac{1}{15} \tan ^{-1}\left(\mathrm{v}_{2}\right) \\
& +\frac{\mathfrak{t}}{40} \frac{\sin ^{2}\left(\mathrm{v}_{3}\right)}{5+\sin ^{2}\left(\mathrm{v}_{3}\right)}+\frac{3 \mathfrak{t}}{20} \frac{\left|\sin ^{-1}\left(\mathrm{v}_{4}\right)\right|}{8+\left|\sin ^{-1}\left(\mathrm{v}_{4}\right)\right|}
\end{aligned}
$$

Thus we can rewrite the above system as

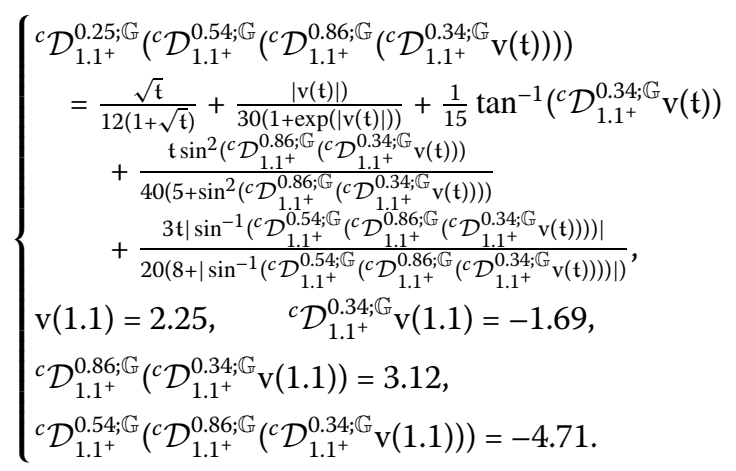


Now we have

$$
\begin{aligned}
& \left|h\left(t, v_{1}(\mathfrak{t}), v_{2}(\mathfrak{t}), \mathrm{v}_{3}(\mathfrak{t}), \mathrm{v}_{4}(\mathfrak{t})\right)-h\left(\mathfrak{t}, \mathrm{v}_{1}^{*}(\mathfrak{t}), \mathrm{v}_{2}^{*}(\mathfrak{t}), \mathrm{v}_{3}^{*}(\mathfrak{t}), \mathrm{v}_{4}^{*}(\mathfrak{t})\right)\right| \\
& =\mid \frac{\left|\mathrm{v}_{1}(\mathfrak{t})\right|}{30\left(1+\exp \left(\mid \mathrm{v}_{1}(\mathfrak{t})\right) \mid\right)}+\frac{1}{15} \tan ^{-1}\left(\mathrm{v}_{2}(\mathfrak{t})\right) \\
& +\frac{\mathfrak{t} \sin ^{2}\left(\mathrm{v}_{3}(\mathfrak{t})\right)}{40\left(5+\sin ^{2}\left(\mathrm{v}_{3}(\mathfrak{t})\right)\right)}+\frac{3 \mathfrak{t}\left|\sin ^{-1}\left(\mathrm{v}_{4}(\mathfrak{t})\right)\right|}{20\left(8+\left|\sin ^{-1}\left(\mathrm{v}_{4}(\mathfrak{t})\right)\right|\right)} \\
& -\left(\frac{\left|\mathrm{v}_{1}^{*}(\mathfrak{t})\right|}{30\left(1+\exp \left(\mid \mathrm{v}_{1}^{*}(\mathfrak{t})\right) \mid\right)}+\frac{1}{15} \tan ^{-1}\left(\mathrm{v}_{2}^{*}(\mathfrak{t})\right)\right. \\
& \left.+\frac{\mathfrak{t} \sin ^{2}\left(\mathrm{v}_{3}^{*}(\mathfrak{t})\right)}{40\left(5+\sin ^{2}\left(\mathrm{v}_{3}^{*}(\mathfrak{t})\right)\right)}+\frac{3 \mathfrak{t}\left|\sin ^{-1}\left(\mathrm{v}_{4}^{*}(\mathfrak{t})\right)\right|}{20\left(8+\left|\sin ^{-1}\left(\mathrm{v}_{4}^{*}(\mathfrak{t})\right)\right|\right)}\right) \mid \\
& \leq \frac{1}{30}\left|\frac{\left|\mathrm{v}_{1}(\mathfrak{t})\right|}{1+\exp \left(\mid \mathrm{v}_{1}(\mathfrak{t})\right) \mid}-\frac{\left|\mathrm{v}_{1}^{*}(\mathfrak{t})\right|}{1+\exp \left(\mid \mathrm{v}_{1}^{*}(\mathfrak{t})\right) \mid}\right| \\
& +\frac{1}{15}\left|\tan ^{-1}\left(\mathrm{v}_{2}(\mathfrak{t})\right)-\tan ^{-1}\left(\mathrm{v}_{2}^{*}(\mathfrak{t})\right)\right| \\
& +\frac{|\mathfrak{t}|}{40}\left|\frac{\sin ^{2}\left(\mathrm{v}_{3}(\mathfrak{t})\right)}{5+\sin ^{2}\left(\mathrm{v}_{3}(\mathfrak{t})\right)}-\frac{\sin ^{2}\left(\mathrm{v}_{3}^{*}(\mathfrak{t})\right)}{5+\sin ^{2}\left(\mathrm{v}_{3}^{*}(\mathfrak{t})\right)}\right| \\
& +\frac{3|\mathfrak{t}|}{20}\left|\frac{\left|\sin ^{-1}\left(\mathrm{v}_{4}(\mathfrak{t})\right)\right|}{8+\left|\sin ^{-1}\left(\mathrm{v}_{4}(\mathfrak{t})\right)\right|}-\frac{\left|\sin ^{-1}\left(\mathrm{v}_{4}^{*}(\mathfrak{t})\right)\right|}{8+\left|\sin ^{-1}\left(\mathrm{v}_{4}^{*}(\mathfrak{t})\right)\right|}\right| \\
& \leq \frac{1}{30}\left|\mathrm{v}_{1}(\mathfrak{t})-\mathrm{v}_{1}^{*}(\mathfrak{t})\right|+\frac{1}{15}\left|\mathrm{v}_{2}(\mathfrak{t})-\mathrm{v}_{2}^{*}(\mathfrak{t})\right| \\
& +\frac{|\mathfrak{t}|}{40}\left|\mathrm{v}_{3}(\mathfrak{t})-\mathrm{v}_{3}^{*}(\mathfrak{t})\right|+\frac{3|\mathfrak{t}|}{20}\left|\mathrm{v}_{4}(\mathfrak{t})-\mathrm{v}_{4}^{*}(\mathfrak{t})\right| \\
& \leq \frac{1}{30} \sum_{j=1}^{4}\left|\mathrm{v}_{j}(\mathfrak{t})-\mathrm{v}_{j}^{*}(\mathfrak{t})\right| \text {. }
\end{aligned}
$$

So we can choose $L=\frac{1}{30}$. Additionally,

$$
h_{0}^{*}=\sup _{\mathfrak{t} \in[1.1,2.6]}|h(t, 0,0,0,0)|=\frac{\sqrt{2.6}}{2(1+\sqrt{2.6})}=0.308608 \text {. }
$$

Now we consider four cases for $\mathbb{G}$ :

$$
\mathbb{G}_{1}(\mathfrak{t})=2^{\mathfrak{t}}, \quad \mathbb{G}_{2}(\mathfrak{t})=\mathfrak{t}, \quad \mathbb{G}_{3}(\mathfrak{t})=\ln \mathfrak{t}, \quad \mathbb{G}_{4}(\mathfrak{t})=\sqrt{\mathfrak{t}}
$$

Note that $\mathbb{G}_{2}, \mathbb{G}_{3}$, and $\mathbb{G}_{4}$ give the Caputo, Caputo-Hadamard, and Katugampola (for $\rho=0.5$ ) derivatives. By using equation (12) in the first case $\mathbb{G}_{1}(\mathfrak{t})=2^{\mathfrak{t}}$, we have

$$
\begin{aligned}
\mathcal{O}=\mathcal{O}_{1}:= & \frac{\left(\mathbb{G}_{1}(b)-\mathbb{G}_{1}(a)\right)^{q+p+r+k}}{\Gamma(q+p+r+k+1)}+\frac{\left(\mathbb{G}_{1}(b)-\mathbb{G}_{1}(a)\right)^{p+r+k}}{\Gamma(p+r+k+1)} \\
& +\frac{\left(\mathbb{G}_{1}(b)-\mathbb{G}_{1}(a)\right)^{r+k}}{\Gamma(r+k+1)}+\frac{\left(\mathbb{G}_{1}(b)-\mathbb{G}_{1}(a)\right)^{k}}{\Gamma(k+1)} \\
= & \frac{\left(\mathbb{G}_{1}(2.6)-\mathbb{G}_{1}(1.1)\right)^{1.99}}{\Gamma(2.99)}+\frac{\left(\mathbb{G}_{1}(2.6)-\mathbb{G}_{1}(1.1)\right)^{1.65}}{\Gamma(2.65)}
\end{aligned}
$$


Table 1 Numerical values of $\mathcal{O}_{1}$ and $\Lambda_{1}$ for $\in[1.1,2.6]$ in Example 6.1 when $\mathbb{G}_{1}=2^{\mathfrak{t}}$

\begin{tabular}{lllll}
\hline$t$ & $\mathcal{O}_{1}$ & $L_{1}<1$ & $\mathcal{O}_{1}$ & $\ell_{1} \geq$ \\
\hline 1.10 & 0.000000 & 0.000000 & 11.770000 & 11.770000 \\
1.20 & 0.441466 & 0.014716 & 16.049142 & 16.427116 \\
1.30 & 0.823549 & 0.027452 & 19.031261 & 19.829775 \\
1.40 & 1.316793 & 0.043893 & 22.196803 & 23.640848 \\
1.50 & 1.949409 & 0.064980 & 25.691224 & 28.120080 \\
1.60 & 2.747700 & 0.091590 & 29.597402 & 33.515007 \\
1.70 & 3.740314 & 0.124677 & 33.984940 & 40.144312 \\
1.80 & 4.959615 & 0.165320 & 38.922357 & 48.465235 \\
1.90 & 6.442580 & 0.214753 & 44.481788 & 59.178840 \\
2.00 & 8.231606 & 0.274387 & 50.741485 & 73.430081 \\
2.10 & 10.375358 & 0.345845 & 57.787565 & 93.234045 \\
2.20 & 12.929718 & 0.430991 & 65.715482 & 122.503611 \\
2.30 & 15.958843 & 0.531961 & 74.631461 & 169.978522 \\
2.40 & 19.536380 & 0.651213 & 84.653973 & 259.995318 \\
2.50 & 23.746839 & 0.791561 & 95.915326 & 495.319774 \\
\hline
\end{tabular}

$$
+\frac{\left(\mathbb{G}_{1}(2.6)-\mathbb{G}_{1}(1.1)\right)^{0.79}}{\Gamma(1.79)}+\frac{\left(\mathbb{G}_{1}(2.6)-\mathbb{G}_{1}(1.1)\right)^{0.25}}{\Gamma(1.25)}
$$

$=23.746838$.

Thus $L \mathcal{O}_{1}=0.791561<1$, and $(\mathrm{C} 1)$ holds. Also, using equation (14), we obtain

$$
\begin{aligned}
\Lambda=\Lambda_{1}:= & \left|\mathrm{v}_{0}\right|+\left|\mathrm{v}_{1}\right|\left(1+\frac{\left(\mathbb{G}_{1}(b)-\mathbb{G}_{1}(a)\right)^{q}}{\Gamma(q+1)}\right) \\
& +\left|\mathrm{v}_{2}\right|\left(1+\frac{\left(\mathbb{G}_{1}(b)-\mathbb{G}_{1}(a)\right)^{p}}{\Gamma(p+1)}+\frac{\left(\mathbb{G}_{1}(b)-\mathbb{G}_{1}(a)\right)^{q+p}}{\Gamma(q+p+1)}\right) \\
& +\left|\mathrm{v}_{3}\right|\left(1+\frac{\left(\mathbb{G}_{1}(b)-\mathbb{G}_{1}(a)\right)^{r}}{\Gamma(r+1)}+\frac{\left(\mathbb{G}_{1}(b)-\mathbb{G}_{1}(a)\right)^{r+p}}{\Gamma(r+p+1)}\right. \\
& \left.+\frac{\left(\mathbb{G}_{1}(b)-\mathbb{G}_{1}(a)\right)^{q+p+r}}{\Gamma(q+p+r+1)}\right) \\
= & |2.25|+|1.69|\left(1+\frac{\left(\mathbb{G}_{1}(2.6)-\mathbb{G}_{1}(1.1)\right)^{0.34}}{\Gamma(1.34)}\right) \\
& +|3.12|\left(1+\frac{\left(\mathbb{G}_{1}(2.6)-\mathbb{G}_{1}(1.1)\right)^{0.86}}{\Gamma(1.86)}+\frac{\left(\mathbb{G}_{1}(2.6)-\mathbb{G}_{1}(1.1)\right)^{1.2}}{\Gamma(2.2)}\right) \\
& +|4.71|\left(1+\frac{\left(\mathbb{G}_{1}(2.6)-\mathbb{G}_{1}(1.1)\right)^{0.54}}{\Gamma(1.54)}+\frac{\left(\mathbb{G}_{1}(2.6)-\mathbb{G}_{1}(1.1)\right)^{1.4}}{\Gamma(2.4)}\right. \\
& \left.+\frac{\left(\mathbb{G}_{1}(2.6)-\mathbb{G}_{1}(1.1)\right)^{1.74}}{\Gamma(2.74)}\right)=95.915326 .
\end{aligned}
$$

Hence

$$
\ell_{1} \geq \frac{\Lambda_{1}+h_{0}^{*} \mathcal{O}_{1}}{1-L \mathcal{O}_{1}}=\frac{95.915326+0.308608 \times 23.746838}{1-0.791561}=493.529331
$$

Table 1 shows the numerical results of $\mathcal{O}_{1}, \Lambda_{1}$, and $\ell_{1}$ for $\mathfrak{t} \in[1.1,2.6]$. These values are also shown in Fig. 1. 


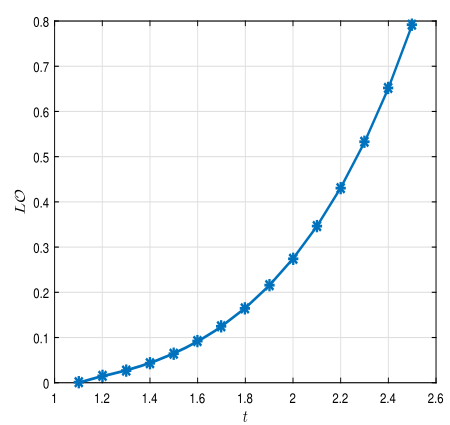

(a) $L \mathcal{O}_{1}$

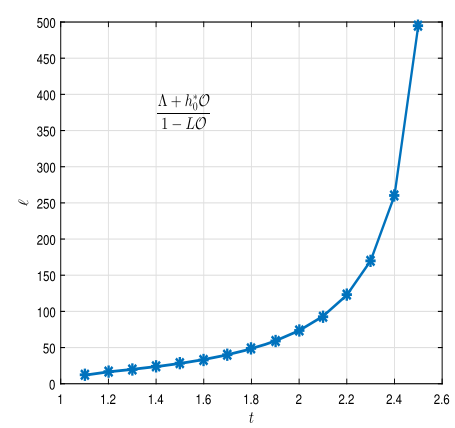

(b) $\ell_{1}$

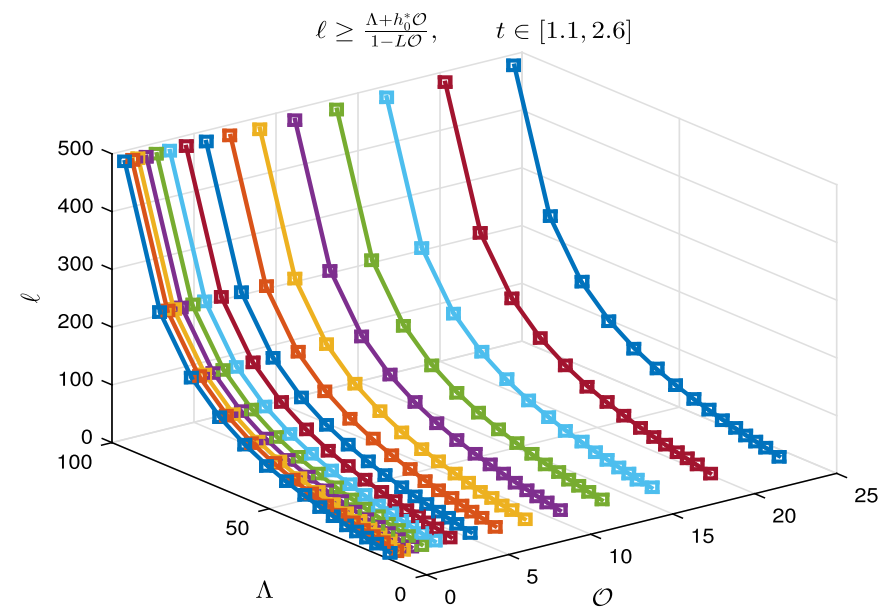

(c) $\ell_{1}$ with $\mathcal{O}_{1}$ and $\Lambda_{1}$

Figure 1 Graphical representation of $L \mathcal{O}_{1}$ and $\ell_{1}$ for $\mathfrak{t} \in[0,2]$ in Example 6.1

In the second case $\mathbb{G}_{2}(\mathfrak{t})=\mathfrak{t}($ Caputo type), we have

$$
\begin{aligned}
\mathcal{O}=\mathcal{O}_{2}:= & \frac{\left(\mathbb{G}_{2}(b)-\mathbb{G}_{2}(a)\right)^{q+p+r+k}}{\Gamma(q+p+r+k+1)}+\frac{\left(\mathbb{G}_{2}(b)-\mathbb{G}_{2}(a)\right)^{p+r+k}}{\Gamma(p+r+k+1)} \\
& +\frac{\left(\mathbb{G}_{2}(b)-\mathbb{G}_{2}(a)\right)^{r+k}}{\Gamma(r+k+1)}+\frac{\left(\mathbb{G}_{2}(b)-\mathbb{G}_{2}(a)\right)^{k}}{\Gamma(k+1)} \\
= & \frac{\left(\mathbb{G}_{2}(2.6)-\mathbb{G}_{2}(1.1)\right)^{1.99}}{\Gamma(2.99)}+\frac{\left(\mathbb{G}_{2}(2.6)-\mathbb{G}_{2}(1.1)\right)^{1.65}}{\Gamma(2.65)} \\
& +\frac{\left(\mathbb{G}_{2}(2.6)-\mathbb{G}_{2}(1.1)\right)^{0.79}}{\Gamma(1.79)}+\frac{\left(\mathbb{G}_{2}(2.6)-\mathbb{G}_{2}(1.1)\right)^{0.25}}{\Gamma(1.25)} \\
= & 5.306821 .
\end{aligned}
$$

Thus $L \mathcal{O}_{2}=0.176894<1$, and (C1) holds. Also, using equation (14), we obtain

$$
\begin{aligned}
\Lambda=\Lambda_{2}:= & \left|\mathrm{v}_{0}\right|+\left|\mathrm{v}_{1}\right|\left(1+\frac{\left(\mathbb{G}_{1}(b)-\mathbb{G}_{1}(a)\right)^{q}}{\Gamma(q+1)}\right) \\
& +\left|\mathrm{v}_{2}\right|\left(1+\frac{\left(\mathbb{G}_{1}(b)-\mathbb{G}_{1}(a)\right)^{p}}{\Gamma(p+1)}+\frac{\left(\mathbb{G}_{1}(b)-\mathbb{G}_{1}(a)\right)^{q+p}}{\Gamma(q+p+1)}\right)
\end{aligned}
$$




$$
\begin{aligned}
+ & \left|\mathrm{v}_{3}\right|\left(1+\frac{\left(\mathbb{G}_{1}(b)-\mathbb{G}_{1}(a)\right)^{r}}{\Gamma(r+1)}+\frac{\left(\mathbb{G}_{1}(b)-\mathbb{G}_{1}(a)\right)^{r+p}}{\Gamma(r+p+1)}\right. \\
+ & \left.\frac{\left(\mathbb{G}_{1}(b)-\mathbb{G}_{1}(a)\right)^{q+p+r}}{\Gamma(q+p+r+1)}\right) \\
= & |2.25|+|1.69|\left(1+\frac{\left(\mathbb{G}_{1}(2.6)-\mathbb{G}_{1}(1.1)\right)^{0.34}}{\Gamma(1.34)}\right) \\
+ & |3.12|\left(1+\frac{\left(\mathbb{G}_{1}(2.6)-\mathbb{G}_{1}(1.1)\right)^{0.86}}{\Gamma(1.86)}\right. \\
+ & \left.\frac{\left(\mathbb{G}_{1}(2.6)-\mathbb{G}_{1}(1.1)\right)^{1.2}}{\Gamma(2.2)}\right) \\
+ & |4.71|\left(1+\frac{\left(\mathbb{G}_{1}(2.6)-\mathbb{G}_{1}(1.1)\right)^{0.54}}{\Gamma(1.54)}\right. \\
+ & \frac{\left(\mathbb{G}_{1}(2.6)-\mathbb{G}_{1}(1.1)\right)^{1.4}}{\Gamma(2.4)} \\
+ & \left.\frac{\left(\mathbb{G}_{1}(2.6)-\mathbb{G}_{1}(1.1)\right)^{1.74}}{\Gamma(2.74)}\right)=40.261437 .
\end{aligned}
$$

Hence

$$
\ell_{2} \geq \frac{\Lambda_{2}+h_{0}^{*} \mathcal{O}_{2}}{1-L \mathcal{O}_{2}}=\frac{40.261437+0.308608 \times 5.306821}{1-0.176894}=50.802414
$$

In the third case $\mathbb{G}_{3}(\mathfrak{t})=\ln \mathfrak{t}($ Caputo-Hadamard type), we have

$$
\begin{aligned}
\mathcal{O}=\mathcal{O}_{3}:= & \frac{\left(\mathbb{G}_{3}(b)-\mathbb{G}_{3}(a)\right)^{q+p+r+k}}{\Gamma(q+p+r+k+1)}+\frac{\left(\mathbb{G}_{3}(b)-\mathbb{G}_{3}(a)\right)^{p+r+k}}{\Gamma(p+r+k+1)} \\
& +\frac{\left(\mathbb{G}_{3}(b)-\mathbb{G}_{3}(a)\right)^{r+k}}{\Gamma(r+k+1)}+\frac{\left(\mathbb{G}_{3}(b)-\mathbb{G}_{3}(a)\right)^{k}}{\Gamma(k+1)} \\
= & \frac{\left(\mathbb{G}_{3}(2.6)-\mathbb{G}_{3}(1.1)\right)^{1.99}}{\Gamma(2.99)}+\frac{\left(\mathbb{G}_{3}(2.6)-\mathbb{G}_{3}(1.1)\right)^{1.65}}{\Gamma(2.65)} \\
& +\frac{\left(\mathbb{G}_{3}(2.6)-\mathbb{G}_{3}(1.1)\right)^{0.79}}{\Gamma(1.79)}+\frac{\left(\mathbb{G}_{3}(2.6)-\mathbb{G}_{3}(1.1)\right)^{0.25}}{\Gamma(1.25)} \\
= & 2.4709 .
\end{aligned}
$$

Thus $L \mathcal{O}_{3}=0.082363<1$, and (C1) holds. Also, using equation (14), we obtain

$$
\begin{aligned}
\Lambda=\Lambda_{3}:= & \left|\mathrm{v}_{0}\right|+\left|\mathrm{v}_{1}\right|\left(1+\frac{\left(\mathbb{G}_{3}(b)-\mathbb{G}_{3}(a)\right)^{q}}{\Gamma(q+1)}\right) \\
& +\left|\mathrm{v}_{2}\right|\left(1+\frac{\left(\mathbb{G}_{3}(b)-\mathbb{G}_{3}(a)\right)^{p}}{\Gamma(p+1)}+\frac{\left(\mathbb{G}_{3}(b)-\mathbb{G}_{3}(a)\right)^{q+p}}{\Gamma(q+p+1)}\right) \\
& +\left|\mathrm{v}_{3}\right|\left(1+\frac{\left(\mathbb{G}_{3}(b)-\mathbb{G}_{3}(a)\right)^{r}}{\Gamma(r+1)}+\frac{\left(\mathbb{G}_{3}(b)-\mathbb{G}_{3}(a)\right)^{r+p}}{\Gamma(r+p+1)}\right. \\
& \left.+\frac{\left(\mathbb{G}_{3}(b)-\mathbb{G}_{3}(a)\right)^{q+p+r}}{\Gamma(q+p+r+1)}\right) \\
= & |2.25|+|1.69|\left(1+\frac{\left(\mathbb{G}_{3}(2.6)-\mathbb{G}_{3}(1.1)\right)^{0.34}}{\Gamma(1.34)}\right)
\end{aligned}
$$




$$
\begin{aligned}
& +|3.12|\left(1+\frac{\left(\mathbb{G}_{3}(2.6)-\mathbb{G}_{3}(1.1)\right)^{0.86}}{\Gamma(1.86)}\right. \\
& \left.+\frac{\left(\mathbb{G}_{3}(2.6)-\mathbb{G}_{3}(1.1)\right)^{1.2}}{\Gamma(2.2)}\right) \\
& +|4.71|\left(1+\frac{\left(\mathbb{G}_{3}(2.6)-\mathbb{G}_{3}(1.1)\right)^{0.54}}{\Gamma(1.54)}\right. \\
& +\frac{\left(\mathbb{G}_{3}(2.6)-\mathbb{G}_{3}(1.1)\right)^{1.4}}{\Gamma(2.4)} \\
& \left.+\frac{\left(\mathbb{G}_{3}(2.6)-\mathbb{G}_{3}(1.1)\right)^{1.74}}{\Gamma(2.74)}\right)=28.290416 .
\end{aligned}
$$

Hence

$$
\ell_{3} \geq \frac{\Lambda_{3}+h_{0}^{*} \mathcal{O}_{3}}{1-L \mathcal{O}_{3}}=\frac{28.290416+0.308608 \times 5.306821}{1-0.082363}=31.660634
$$

In the fourth case $\mathbb{G}_{4}(\mathfrak{t})=\sqrt{\mathfrak{t}}($ Katugampola type for $\rho=0.5)$, we have

$$
\begin{aligned}
\mathcal{O}=\mathcal{O}_{4}:= & \frac{\left(\mathbb{G}_{4}(b)-\mathbb{G}_{4}(a)\right)^{q+p+r+k}}{\Gamma(q+p+r+k+1)}+\frac{\left(\mathbb{G}_{4}(b)-\mathbb{G}_{4}(a)\right)^{p+r+k}}{\Gamma(p+r+k+1)} \\
& +\frac{\left(\mathbb{G}_{4}(b)-\mathbb{G}_{4}(a)\right)^{r+k}}{\Gamma(r+k+1)}+\frac{\left(\mathbb{G}_{4}(b)-\mathbb{G}_{4}(a)\right)^{k}}{\Gamma(k+1)} \\
= & \frac{\left(\mathbb{G}_{4}(2.6)-\mathbb{G}_{4}(1.1)\right)^{1.99}}{\Gamma(2.99)}+\frac{\left(\mathbb{G}_{4}(2.6)-\mathbb{G}_{4}(1.1)\right)^{1.65}}{\Gamma(2.65)} \\
& +\frac{\left(\mathbb{G}_{4}(2.6)-\mathbb{G}_{4}(1.1)\right)^{0.79}}{\Gamma(1.79)}+\frac{\left(\mathbb{G}_{4}(2.6)-\mathbb{G}_{4}(1.1)\right)^{0.25}}{\Gamma(1.25)} \\
= & 1.43141 .
\end{aligned}
$$

Thus $L \mathcal{O}_{4}=0.047713<1$, and (C1) holds. Also, using equation (14), we obtain

$$
\begin{aligned}
\Lambda=\Lambda_{4}:= & \left|\mathrm{v}_{0}\right|+\left|\mathrm{v}_{1}\right|\left(1+\frac{\left(\mathbb{G}_{4}(b)-\mathbb{G}_{4}(a)\right)^{q}}{\Gamma(q+1)}\right) \\
& +\left|\mathrm{v}_{2}\right|\left(1+\frac{\left(\mathbb{G}_{4}(b)-\mathbb{G}_{4}(a)\right)^{p}}{\Gamma(p+1)}+\frac{\left(\mathbb{G}_{4}(b)-\mathbb{G}_{4}(a)\right)^{q+p}}{\Gamma(q+p+1)}\right) \\
& +\left|\mathrm{v}_{3}\right|\left(1+\frac{\left(\mathbb{G}_{4}(b)-\mathbb{G}_{4}(a)\right)^{r}}{\Gamma(r+1)}+\frac{\left(\mathbb{G}_{4}(b)-\mathbb{G}_{4}(a)\right)^{r+p}}{\Gamma(r+p+1)}\right. \\
& \left.+\frac{\left(\mathbb{G}_{4}(b)-\mathbb{G}_{4}(a)\right)^{q+p+r}}{\Gamma(q+p+r+1)}\right) \\
= & |2.25|+|1.69|\left(1+\frac{\left(\mathbb{G}_{4}(2.6)-\mathbb{G}_{4}(1.1)\right)^{0.34}}{\Gamma(1.34)}\right) \\
& +|3.12|\left(1+\frac{\left(\mathbb{G}_{4}(2.6)-\mathbb{G}_{4}(1.1)\right)^{0.86}}{\Gamma(1.86)}+\frac{\left(\mathbb{G}_{4}(2.6)-\mathbb{G}_{4}(1.1)\right)^{1.2}}{\Gamma(2.2)}\right) \\
& +|4.71|\left(1+\frac{\left(\mathbb{G}_{4}(2.6)-\mathbb{G}_{4}(1.1)\right)^{0.54}}{\Gamma(1.54)}+\frac{\left(\mathbb{G}_{4}(2.6)-\mathbb{G}_{4}(1.1)\right)^{1.4}}{\Gamma(2.4)}\right. \\
& \left.+\frac{\left(\mathbb{G}_{4}(2.6)-\mathbb{G}_{4}(1.1)\right)^{1.74}}{\Gamma(2.74)}\right)=22.866749 .
\end{aligned}
$$


Table 2 Numerical values of $\mathcal{O}_{j}$ and $\Lambda_{j}, j=2,3,4$, for $\mathfrak{t} \in[1.1,2.6]$ in Example 6.1 when $\mathbb{G}_{2}=\mathfrak{t}$, $\mathbb{G}_{3}=\ln \mathfrak{t}$, and $\mathbb{G}_{4}=\sqrt{\mathfrak{t}}$

\begin{tabular}{|c|c|c|c|c|}
\hline$t$ & $\mathcal{O}_{1}$ & $L \mathcal{O}_{1}<1$ & $\mathcal{O}_{1}$ & $\ell_{1} \geq$ \\
\hline \multicolumn{5}{|c|}{$\overline{\mathbb{G}_{2}(\mathfrak{t})=\mathfrak{t}}$} \\
\hline 1.10 & 0.0000 & 0.0000 & 11.7700 & 11.7700 \\
\hline 1.20 & 0.3282 & 0.0109 & 15.0025 & 15.2709 \\
\hline 1.30 & 0.5418 & 0.0181 & 16.9019 & 17.3831 \\
\hline 1.40 & 0.7766 & 0.0259 & 18.6975 & 19.4404 \\
\hline 1.50 & 1.0389 & 0.0346 & 20.4797 & 21.5464 \\
\hline 1.60 & 1.3307 & 0.0444 & 22.2789 & 23.7427 \\
\hline 1.70 & 1.6525 & 0.0551 & 24.1088 & 26.0539 \\
\hline 1.80 & 2.0046 & 0.0668 & 25.9761 & 28.4991 \\
\hline 1.90 & 2.3869 & 0.0796 & 27.8846 & 31.0952 \\
\hline 2.00 & 2.7993 & 0.0933 & 29.8361 & 33.8594 \\
\hline 2.10 & 3.2416 & 0.1081 & 31.8319 & 36.8096 \\
\hline 2.20 & 3.7137 & 0.1238 & 33.8722 & 39.9656 \\
\hline 2.30 & 4.2154 & 0.1405 & 35.9573 & 43.3493 \\
\hline 2.40 & 4.7465 & 0.1582 & 38.0871 & 46.9858 \\
\hline 2.50 & 5.3068 & 0.1769 & 40.2614 & 50.9037 \\
\hline \multicolumn{5}{|c|}{$\mathbb{G}_{3}(\mathfrak{t})=\ln \mathfrak{t}$} \\
\hline 1.10 & 0.0000 & 0.0000 & 11.7700 & 11.7700 \\
\hline 1.20 & 0.3010 & 0.0100 & 14.7349 & 14.9780 \\
\hline 1.30 & 0.4698 & 0.0157 & 16.2959 & 16.7025 \\
\hline 1.40 & 0.6354 & 0.0212 & 17.6460 & 18.2281 \\
\hline 1.50 & 0.8019 & 0.0267 & 18.8784 & 19.6511 \\
\hline 1.60 & 0.9698 & 0.0323 & 20.0278 & 21.0062 \\
\hline 1.70 & 1.1385 & 0.0380 & 21.1123 & 22.3103 \\
\hline 1.80 & 1.3077 & 0.0436 & 22.1426 & 23.5737 \\
\hline 1.90 & 1.4767 & 0.0492 & 23.1263 & 24.8029 \\
\hline 2.00 & 1.6452 & 0.0548 & 24.0688 & 26.0026 \\
\hline 2.10 & 1.8129 & 0.0604 & 24.9745 & 27.1763 \\
\hline 2.20 & 1.9795 & 0.0660 & 25.8469 & 28.3269 \\
\hline 2.30 & 2.1448 & 0.0715 & 26.6887 & 29.4566 \\
\hline 2.40 & 2.3086 & 0.0770 & 27.5025 & 30.5673 \\
\hline 2.50 & 2.4709 & 0.0824 & 28.2904 & 31.6606 \\
\hline \multicolumn{5}{|c|}{$\mathbb{G}_{4}(\mathfrak{t})=\sqrt{\mathfrak{t}}$} \\
\hline 1.10 & 0.0000 & 0.0000 & 11.7700 & 11.7700 \\
\hline 1.20 & 0.2130 & 0.0071 & 13.8243 & 13.9894 \\
\hline 1.30 & 0.3101 & 0.0103 & 14.8256 & 15.0771 \\
\hline 1.40 & 0.4003 & 0.0133 & 15.6800 & 16.0172 \\
\hline 1.50 & 0.4890 & 0.0163 & 16.4605 & 16.8867 \\
\hline 1.60 & 0.5779 & 0.0193 & 17.1943 & 17.7139 \\
\hline 1.70 & 0.6678 & 0.0223 & 17.8948 & 18.5129 \\
\hline 1.80 & 0.7589 & 0.0253 & 18.5698 & 19.2920 \\
\hline 1.90 & 0.8512 & 0.0284 & 19.2245 & 20.0562 \\
\hline 2.00 & 0.9449 & 0.0315 & 19.8622 & 20.8092 \\
\hline 2.10 & 1.0398 & 0.0347 & 20.4856 & 21.5535 \\
\hline 2.20 & 1.1360 & 0.0379 & 21.0964 & 22.2910 \\
\hline 2.30 & 1.2333 & 0.0411 & 21.6961 & 23.0232 \\
\hline 2.40 & 1.3318 & 0.0444 & 22.2859 & 23.7513 \\
\hline 2.50 & 1.4314 & 0.0477 & 22.8668 & 24.4764 \\
\hline
\end{tabular}

Hence

$$
\ell_{4} \geq \frac{\Lambda_{4}+h_{0}^{*} \mathcal{O}_{4}}{1-L \mathcal{O}_{4}}=\frac{22.866749+0.308608 \times 1.43141}{1-0.047713}=24.476352
$$

Table 2 shows the numerical values of $\mathcal{O}_{j}, \Lambda_{j}$, and $\ell_{j}, j=2,3,4$, for $\mathfrak{t} \in[1.1,2.6]$. These values are also shown in Fig. 2. Figure 3 shows a 3D-graph of the numerical values of $\ell_{j}$ based on $\mathcal{O}_{j}$ and $\Lambda_{j}, j=2,3,4$, for $\mathfrak{t} \in[1.1,2.6]$. 


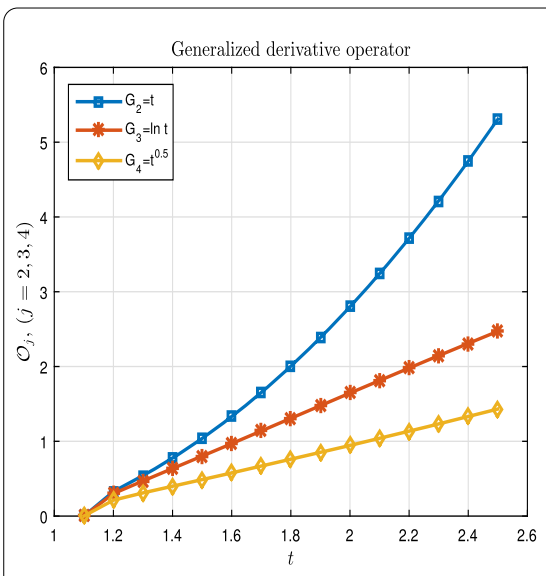

(a) $\mathcal{O}_{j}(j=2,3,4)$

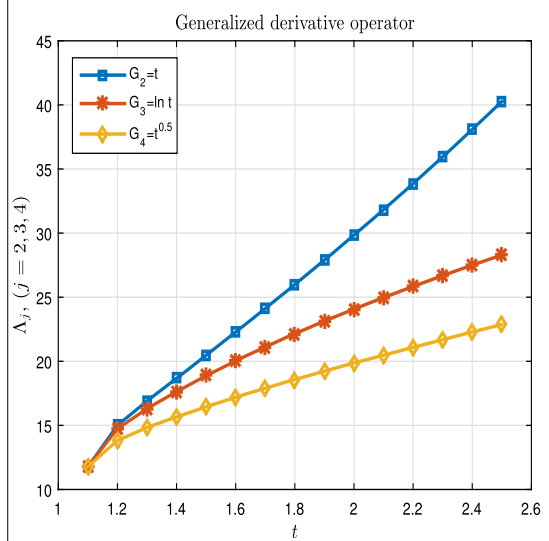

(c) $\Lambda_{j}(j=2,3,4)$

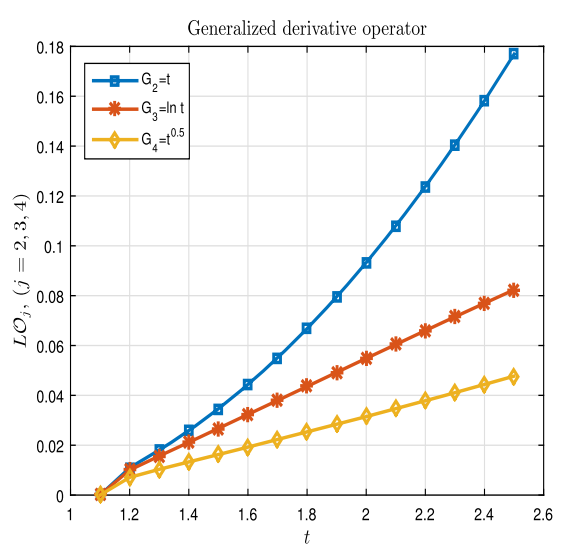

(b) $L \mathcal{O}_{j}(j=2,3,4)$

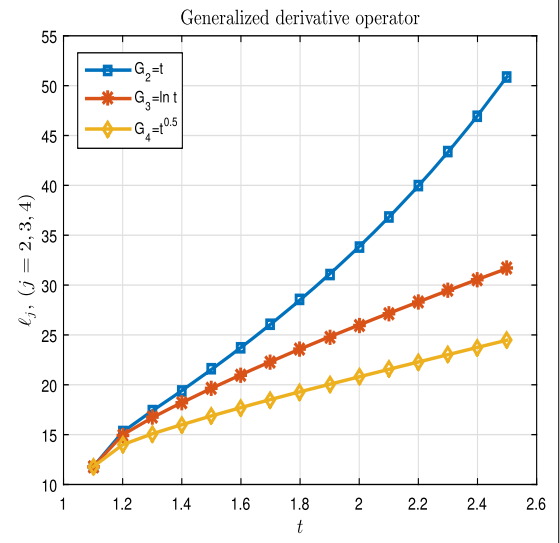

(d) $\ell_{j}(j=2,3,4)$

Figure 2 Graphical representation of $\mathcal{O}_{j}, \Lambda_{j}$, and $\ell_{j}$ for $\mathfrak{t} \in[1.1,2.6]$ and $j=2,3,4$ in Example 6.1 where $\mathbb{G}_{2}(t)=\mathfrak{t}, \mathbb{G}_{3}(\mathfrak{t})=\ln \mathfrak{t}$, and $\mathbb{G}_{4}(\mathfrak{t})=\sqrt{\mathfrak{t}}$

In all four cases for the function $\mathbb{G}$, we saw that all requirements of Theorem 3.2 are fulfilled. Therefore this guarantees that for all four different cases in terms of the function $\mathbb{G}$, the fractional $\mathbb{G}$-snap system (56) admits a unique solution on the interval $[1.1,2.6]$.

In the next example, we examine the correctness of the results caused by Theorem 3.3. In that example, we consider the case $\mathbb{G}(\mathfrak{t})=\mathfrak{t}$ (Caputo type) for three different orders $q_{1}$, $q_{2}$, and $q_{3}$ and show the obtained results computationally and graphically.

Example 6.2 Based on the given system (4) for $\mathbb{G}(\mathfrak{t})=\mathfrak{t}$ (Caputo type), we consider the nonlinear fractional $\mathbb{G}$-snap BVP

$$
\left\{\begin{array}{l}
{ }^{C} \mathcal{D}_{0.02^{+}}^{q ; \mathbb{G}} \mathrm{v}(\mathfrak{t})=\mathrm{u}(\mathfrak{t}), \quad 0.02 \leq \mathfrak{t} \leq 0.99, \mathrm{v}(0.02)=-1.07 \\
{ }^{c} \mathcal{D}_{0.02^{+}}^{0.077 ;} \mathrm{u}(\mathfrak{t})=\mathrm{w}(\mathfrak{t}), \quad \mathrm{u}(0.02)=4.46 \\
{ }^{c} \mathcal{D}_{0.02^{+}}^{0.27 ; \mathbb{G}} \mathrm{w}(\mathfrak{t})=\mathrm{x}(\mathfrak{t}), \quad \mathrm{w}(0.02)=-3.8 \\
{ }^{c} \mathcal{D}_{0.02^{+}}^{0.03 ;} \mathrm{G}(\mathfrak{t})=h(\mathfrak{t}, \mathrm{v}, \mathrm{u}, \mathrm{w}, \mathrm{x}), \quad \mathrm{x}(1.1)=-2.15,
\end{array}\right.
$$




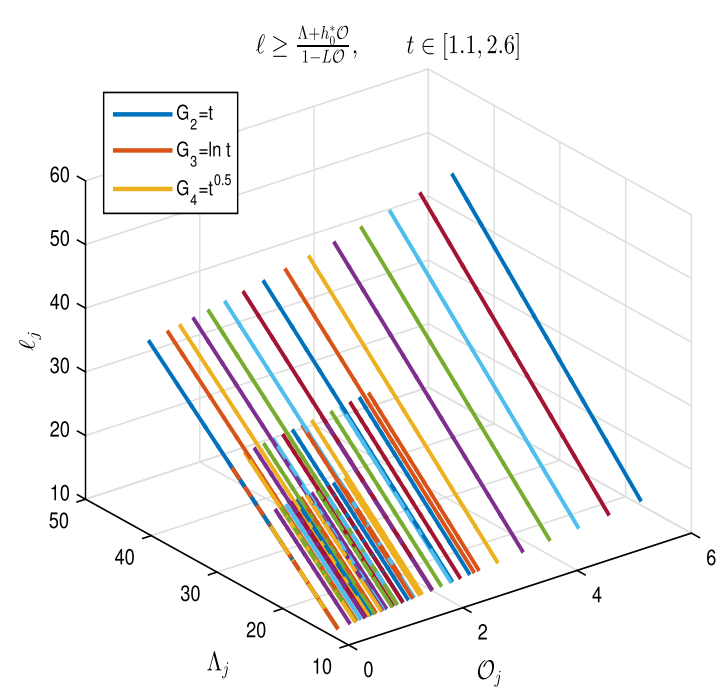

Figure $33 \mathrm{D}$-graph of $\ell \geq \frac{\Lambda+h_{0}^{*} \mathcal{O}}{1-L \mathcal{O}}$ for $\mathfrak{t} \in[1.1,2.6]$ in Example 6.1

where

$$
\begin{aligned}
h(\mathfrak{t}, \mathrm{v}, \mathrm{u}, \mathrm{w}, \mathrm{x})= & \frac{\sin (\mathrm{v}(\mathfrak{t}))}{10(25+\sin (\mathrm{v}(\mathfrak{t})))}+\frac{\tan ^{-1}(\mathrm{u}(\mathfrak{t}))}{15\left(32+\mathfrak{t}^{2}\right)} \\
& +\frac{\mathfrak{t}(\mathrm{w}(\mathfrak{t}))^{2}}{14\left(17+(\mathrm{w}(\mathfrak{t}))^{2}\right)}+\frac{3 \mathfrak{t}\left|\sin ^{-1}(\mathrm{x}(\mathfrak{t}))\right|}{\left(10+3 \mathfrak{t}^{2}\right)\left(13+\left|\sin ^{-1}(\mathrm{x}(\mathfrak{t}))\right|\right)}
\end{aligned}
$$

for $\mathfrak{t} \in[0.02,0.99]$. Clearly, $a=0.02, b=0.99, \mathrm{v}(0)=\mathrm{v}_{0}=-1.07, p=0.37 \in(0,1], \mathrm{u}(0)=\mathrm{v}_{1}=$ 4.46, $r=0.27 \in(0,1], \mathrm{w}(0)=\mathrm{v}_{2}=-3.8, k=0.8 \in(0,1], \mathrm{x}(0)=\mathrm{v}_{3}=-2.15$, and

$$
\begin{aligned}
h\left(\mathfrak{t}, \mathrm{v}_{1}, \mathrm{v}_{2}, \mathrm{v}_{3}, \mathrm{v}_{3}\right)= & \frac{\sin \left(\mathrm{v}_{1}(\mathfrak{t})\right)}{10\left(25+\sin \left(\mathrm{v}_{1}(\mathfrak{t})\right)\right)}+\frac{\tan ^{-1}\left(\mathrm{v}_{2}(\mathfrak{t})\right)}{15\left(32+\mathfrak{t}^{2}\right)} \\
& +\frac{\mathfrak{t}\left(\mathrm{v}_{3}(\mathfrak{t})\right)^{2}}{14\left(17+\left(\mathrm{v}_{3}(\mathfrak{t})\right)^{2}\right)}+\frac{3 \mathfrak{t}\left|\sin ^{-1}\left(\mathrm{v}_{4}(\mathfrak{t})\right)\right|}{\left(10+3 \mathfrak{t}^{2}\right)\left(13+\left|\sin ^{-1}\left(\mathrm{v}_{4}(\mathfrak{t})\right)\right|\right)}
\end{aligned}
$$

for $\mathfrak{t} \in[0.02,0.99]$. Thus we can rewrite the above system as

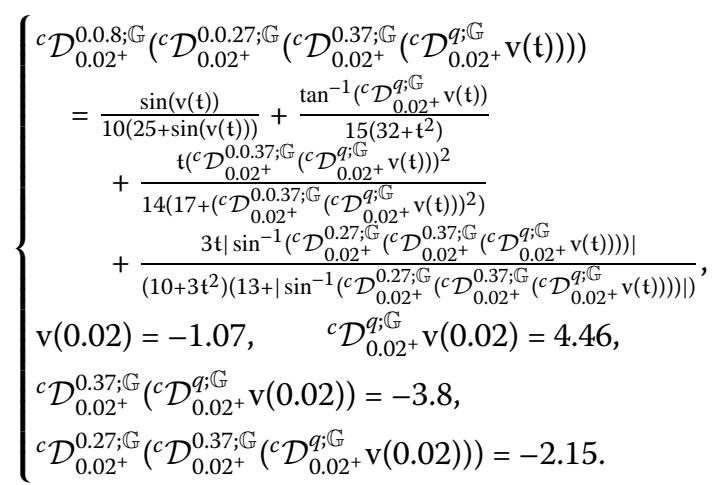

Now we have

$$
\left|h\left(\mathrm{t}, \mathrm{v}_{1}, \mathrm{v}_{2}, \mathrm{v}_{3}, \mathrm{v}_{3}\right)\right|
$$




$$
\begin{aligned}
= & \mid \frac{\sin \left(\mathrm{v}_{1}(\mathfrak{t})\right)}{10\left(25+\sin \left(\mathrm{v}_{1}(\mathfrak{t})\right)\right)}+\frac{\tan ^{-1}\left(\mathrm{v}_{2}(\mathfrak{t})\right)}{15\left(32+\mathfrak{t}^{2}\right)} \\
& +\frac{\mathfrak{t}\left(\mathrm{v}_{3}(\mathfrak{t})\right)^{2}}{14\left(17+\left(\mathrm{v}_{3}(\mathfrak{t})\right)^{2}\right)}+\frac{3 \mathfrak{t}\left|\sin ^{-1}\left(\mathrm{v}_{4}(\mathfrak{t})\right)\right|}{\left(10+3 \mathfrak{t}^{2}\right)\left(13+\left|\sin ^{-1}\left(\mathrm{v}_{4}(\mathfrak{t})\right)\right|\right)} \mid \\
\leq & \frac{1}{10}\left|\frac{\sin \left(\mathrm{v}_{1}(\mathfrak{t})\right)}{25+\sin \left(\mathrm{v}_{1}(\mathfrak{t})\right)}\right|+\frac{1}{15}\left|\frac{\tan ^{-1}\left(\mathrm{v}_{2}(\mathfrak{t})\right)}{32+\mathfrak{t}^{2}}\right| \\
& +\frac{|\mathfrak{t}|}{14}\left|\frac{\left(\mathrm{v}_{3}(\mathfrak{t})\right)^{2}}{17+\left(\mathrm{v}_{3}(\mathfrak{t})\right)^{2}}\right|+\left|\frac{3 \mathfrak{t}}{10+3 \mathfrak{t}^{2}}\right|\left|\frac{\left|\sin ^{-1}\left(\mathrm{v}_{4}(\mathfrak{t})\right)\right|}{13+\left|\sin ^{-1}\left(\mathrm{v}_{4}(\mathfrak{t})\right)\right|}\right| \\
\leq & \frac{\mathfrak{t}}{10}\left(\frac{1}{15}\left|\mathrm{v}_{1}(\mathfrak{t})\right|+\frac{1}{15}\left|\mathrm{v}_{2}(\mathfrak{t})\right|+\frac{1}{15}\left|\mathrm{v}_{3}(\mathfrak{t})\right|+\frac{1}{15}\left|\mathrm{v}_{4}(\mathfrak{t})\right|\right) \\
= & \frac{1}{10} \mathfrak{t} \sum_{j=1}^{4} \frac{1}{15}\left|\mathrm{v}_{j}(\mathfrak{t})\right| .
\end{aligned}
$$

So we can choose $\varrho(\mathfrak{t})=\frac{1}{10} \mathfrak{t}$ and $f(\mathrm{v})=\frac{1}{15} \mathrm{v}$. Thus for $j=1,2,3,4$,

$$
\left|h\left(\mathfrak{t}, \mathrm{v}_{1}(\mathfrak{t}), \mathbf{v}_{2}(\mathfrak{t}), \mathbf{v}_{3}(\mathfrak{t}), \mathrm{v}_{4}(\mathfrak{t})\right)\right| \leq \varrho(\mathfrak{t}) f\left(\sum_{j=1}^{4}\left|\mathrm{v}_{j}(\mathfrak{t})\right|\right),
$$

and (C2) holds. In addition,

$$
\varrho_{0}^{*}=\sup _{\mathfrak{t} \in[0.02,0.99]}|\varrho(\mathfrak{t})|=0.099
$$

Now we consider three cases for $q \in\left\{q_{1}=0.28, q_{2}=0.53, q_{3}=0.89\right\}$. By equation (12), in the first case $q=q_{1}=0.28$, we have

$$
\begin{aligned}
\mathcal{O}=\mathcal{O}_{1}:= & \frac{(\mathbb{G}(b)-\mathbb{G}(a))^{q_{1}+p+r+k}}{\Gamma\left(q_{1}+p+r+k+1\right)}+\frac{(\mathbb{G}(b)-\mathbb{G}(a))^{p+r+k}}{\Gamma(p+r+k+1)} \\
& +\frac{(\mathbb{G}(b)-\mathbb{G}(a))^{r+k}}{\Gamma(r+k+1)}+\frac{(\mathbb{G}(b)-\mathbb{G}(a))^{k}}{\Gamma(k+1)} \\
= & \frac{(\mathbb{G}(0.99)-\mathbb{G}(0.02))^{1.72}}{\Gamma(2.72)}+\frac{(\mathbb{G}(0.99)-\mathbb{G}(0.02))^{1.44}}{\Gamma(2.44)} \\
& +\frac{(\mathbb{G}(0.99)-\mathbb{G}(0.02))^{1.07}}{\Gamma(2.07)}+\frac{(\mathbb{G}(0.99)-\mathbb{G}(0.02))^{0.8}}{\Gamma(1.8)} \\
= & 4.120828 .
\end{aligned}
$$

Also, by equation (14) we obtain

$$
\begin{aligned}
\Lambda=\Lambda_{1}:= & \left|\mathrm{v}_{0}\right|+\left|\mathrm{v}_{1}\right|\left(1+\frac{(\mathbb{G}(b)-\mathbb{G}(a))^{q_{1}}}{\Gamma\left(q_{1}+1\right)}\right) \\
& +\left|\mathrm{v}_{2}\right|\left(1+\frac{(\mathbb{G}(b)-\mathbb{G}(a))^{p}}{\Gamma(p+1)}+\frac{(\mathbb{G}(b)-\mathbb{G}(a))^{q_{1}+p}}{\Gamma\left(q_{1}+p+1\right)}\right) \\
& +\left|\mathrm{v}_{3}\right|\left(1+\frac{(\mathbb{G}(b)-\mathbb{G}(a))^{r}}{\Gamma(r+1)}+\frac{(\mathbb{G}(b)-\mathbb{G}(a))^{r+p}}{\Gamma(r+p+1)}\right. \\
& \left.+\frac{(\mathbb{G}(b)-\mathbb{G}(a))^{q+p+r}}{\Gamma\left(q_{1}+p+r+1\right)}\right)
\end{aligned}
$$




$$
\begin{aligned}
= & |-1.07|+|4.46|\left(1+\frac{(\mathbb{G}(0.99)-\mathbb{G}(0.2))^{0.28}}{\Gamma(1.28)}\right) \\
& +|-3.8|\left(1+\frac{(\mathbb{G}(0.99)-\mathbb{G}(0.02))^{0.37}}{\Gamma(1.37)}\right. \\
& \left.+\frac{(\mathbb{G}(0.99)-\mathbb{G}(0.02))^{0.65}}{\Gamma(1.65)}\right) \\
& +|-2.15|\left(1+\frac{(\mathbb{G}(0.99)-\mathbb{G}(0.02))^{0.27}}{\Gamma(1.27)}\right. \\
& +\frac{(\mathbb{G}(0.99)-\mathbb{G}(0.02))^{0.55}}{\Gamma(1.55)} \\
& \left.+\frac{(\mathbb{G}(0.99)-\mathbb{G}(0.02))^{0.92}}{\Gamma(1.92)}\right)=31.920297 .
\end{aligned}
$$

We consider $B=100$. Then, substituting (69), (70), and (71) into inequality (24), we obtain

$$
\begin{aligned}
\Lambda_{1}+\mathcal{O}_{1} \varrho_{0}^{*} f(B) & =31.920297+4.120828 \times 0.099 \times f(100) \\
& =34.640043<100=B .
\end{aligned}
$$

Hence (C3) holds for $q=q_{1}=0.28$.

In the second case for $q=q_{2}=0.53$, we get

$$
\begin{aligned}
\mathcal{O}=\mathcal{O}_{2}:= & \frac{(\mathbb{G}(b)-\mathbb{G}(a))^{q_{2}+p+r+k}}{\Gamma\left(q_{2}+p+r+k+1\right)}+\frac{(\mathbb{G}(b)-\mathbb{G}(a))^{p+r+k}}{\Gamma(p+r+k+1)} \\
& +\frac{(\mathbb{G}(b)-\mathbb{G}(a))^{r+k}}{\Gamma(r+k+1)}+\frac{(\mathbb{G}(b)-\mathbb{G}(a))^{k}}{\Gamma(k+1)} \\
= & \frac{(\mathbb{G}(0.99)-\mathbb{G}(0.02))^{1.97}}{\Gamma(2.97)}+\frac{(\mathbb{G}(0.99)-\mathbb{G}(0.02))^{1.44}}{\Gamma(2.44)} \\
& +\frac{(\mathbb{G}(0.99)-\mathbb{G}(0.02))^{1.07}}{\Gamma(2.07)}+\frac{(\mathbb{G}(0.99)-\mathbb{G}(0.02))^{0.8}}{\Gamma(1.8)} \\
= & 4.037502 .
\end{aligned}
$$

Also, by equation (14) we obtain

$$
\begin{aligned}
\Lambda=\Lambda_{2}:= & \left|\mathrm{v}_{0}\right|+\left|\mathrm{v}_{1}\right|\left(1+\frac{(\mathbb{G}(b)-\mathbb{G}(a))^{q_{2}}}{\Gamma\left(q_{2}+1\right)}\right) \\
& +\left|\mathrm{v}_{2}\right|\left(1+\frac{(\mathbb{G}(b)-\mathbb{G}(a))^{p}}{\Gamma(p+1)}+\frac{(\mathbb{G}(b)-\mathbb{G}(a))^{q_{2}+p}}{\Gamma\left(q_{2}+p+1\right)}\right) \\
& +\left|\mathrm{v}_{3}\right|\left(1+\frac{(\mathbb{G}(b)-\mathbb{G}(a))^{r}}{\Gamma(r+1)}+\frac{(\mathbb{G}(b)-\mathbb{G}(a))^{r+p}}{\Gamma(r+p+1)}\right. \\
& \left.+\frac{(\mathbb{G}(b)-\mathbb{G}(a))^{q_{2}+p+r}}{\Gamma\left(q_{2}+p+r+1\right)}\right) \\
= & |-1.07|+|4.46|\left(1+\frac{(\mathbb{G}(0.99)-\mathbb{G}(0.2))^{0.53}}{\Gamma(1.53)}\right) \\
& +|-3.8|\left(1+\frac{(\mathbb{G}(0.99)-\mathbb{G}(0.02))^{0.37}}{\Gamma(1.37)}\right.
\end{aligned}
$$




$$
\begin{aligned}
& \left.+\frac{(\mathbb{G}(0.99)-\mathbb{G}(0.02))^{0.9}}{\Gamma(1.9)}\right) \\
& +|-2.15|\left(1+\frac{(\mathbb{G}(0.99)-\mathbb{G}(0.02))^{0.27}}{\Gamma(1.27)}\right. \\
& +\frac{(\mathbb{G}(0.99)-\mathbb{G}(0.02))^{0.8}}{\Gamma(1.8)} \\
& \left.+\frac{(\mathbb{G}(0.99)-\mathbb{G}(0.02))^{1.33}}{\Gamma(2.33)}\right)=31.486714 .
\end{aligned}
$$

We consider $K=100$. Then, substituting (69), (72), and (73) into inequality (24), we obtain

$$
\begin{aligned}
\Lambda_{2}+\mathcal{O}_{2} \varrho_{0}^{*} f(B) & =31.486714+4.037502 \times 0.099 \times f(100) \\
& =34.151466<100=B .
\end{aligned}
$$

Hence (C3) holds for $q=q_{2}=0.53$.

In the third case for $q=q_{3}=0.89$, we get

$$
\begin{aligned}
\mathcal{O}=\mathcal{O}_{3}:= & \frac{(\mathbb{G}(b)-\mathbb{G}(a))^{q_{3}+p+r+k}}{\Gamma\left(q_{3}+p+r+k+1\right)}+\frac{(\mathbb{G}(b)-\mathbb{G}(a))^{p+r+k}}{\Gamma(p+r+k+1)} \\
& +\frac{(\mathbb{G}(b)-\mathbb{G}(a))^{r+k}}{\Gamma(r+k+1)}+\frac{(\mathbb{G}(b)-\mathbb{G}(a))^{k}}{\Gamma(k+1)} \\
= & \frac{(\mathbb{G}(0.99)-\mathbb{G}(0.02))^{2.33}}{\Gamma(3.33)}+\frac{(\mathbb{G}(0.99)-\mathbb{G}(0.02))^{1.44}}{\Gamma(2.44)} \\
& +\frac{(\mathbb{G}(0.99)-\mathbb{G}(0.02))^{1.07}}{\Gamma(2.07)}+\frac{(\mathbb{G}(0.99)-\mathbb{G}(0.02))^{0.8}}{\Gamma(1.8)} \\
= & 3.866648 .
\end{aligned}
$$

Also, using equation (14), we obtain

$$
\begin{aligned}
\Lambda=\Lambda_{3}:= & \left|\mathrm{v}_{0}\right|+\left|\mathrm{v}_{1}\right|\left(1+\frac{(\mathbb{G}(b)-\mathbb{G}(a))^{q_{3}}}{\Gamma\left(q_{3}+1\right)}\right) \\
& +\left|\mathrm{v}_{2}\right|\left(1+\frac{(\mathbb{G}(b)-\mathbb{G}(a))^{p}}{\Gamma(p+1)}+\frac{(\mathbb{G}(b)-\mathbb{G}(a))^{q_{3}+p}}{\Gamma\left(q_{3}+p+1\right)}\right) \\
& +\left|\mathrm{v}_{3}\right|\left(1+\frac{(\mathbb{G}(b)-\mathbb{G}(a))^{r}}{\Gamma(r+1)}+\frac{(\mathbb{G}(b)-\mathbb{G}(a))^{r+p}}{\Gamma(r+p+1)}\right. \\
& \left.+\frac{(\mathbb{G}(b)-\mathbb{G}(a))^{q_{3}+p+r}}{\Gamma\left(q_{3}+p+r+1\right)}\right) \\
= & |-1.07|+|4.46|\left(1+\frac{(\mathbb{G}(0.99)-\mathbb{G}(0.2))^{0.89}}{\Gamma(1.89)}\right) \\
& +|-3.8|\left(1+\frac{(\mathbb{G}(0.99)-\mathbb{G}(0.02))^{0.37}}{\Gamma(1.37)}\right. \\
& \left.+\frac{(\mathbb{G}(0.99)-\mathbb{G}(0.02))^{1.26}}{\Gamma(2.26)}\right) \\
& +|-2.15|\left(1+\frac{(\mathbb{G}(0.99)-\mathbb{G}(0.02))^{0.27}}{\Gamma(1.27)}+\frac{(\mathbb{G}(0.99)-\mathbb{G}(0.02))^{0.8}}{\Gamma(1.8)}\right.
\end{aligned}
$$


Table 3 Numerical results of $\mathcal{O}_{i}$ and $\Lambda_{i}, i=1,2,3$, for $\mathfrak{t} \in[0.02,0.99]$ in Example 6.2 when $q_{1}=0.28$, $q_{2}=0.53$, and $q_{3}=0.89$

\begin{tabular}{llll}
\hline & \multicolumn{2}{l}{$q_{1}=0.28$} & \\
\cline { 2 - 4 } $\mathfrak{t}$ & $\mathcal{O}_{1}$ & $\Lambda_{1}$ & $\frac{B}{\Lambda_{1}+\mathcal{O}_{1} \varrho_{0}^{*} f(B)}>1$ \\
\hline 0.02 & 0.0000 & 11.4800 & 8.7108 \\
0.07 & 0.1417 & 17.1867 & 5.7870 \\
0.12 & 0.2863 & 18.9408 & 5.2275 \\
0.17 & 0.4400 & 20.2643 & 4.8651 \\
0.22 & 0.6024 & 21.3756 & 4.5928 \\
0.27 & 0.7730 & 22.3552 & 4.3734 \\
0.32 & 0.9514 & 23.2432 & 4.1892 \\
0.37 & 1.1372 & 24.0628 & 4.0301 \\
0.42 & 1.3301 & 24.8289 & 3.8900 \\
0.47 & 1.5298 & 25.5518 & 3.7649 \\
0.52 & 1.7361 & 26.2387 & 3.6517 \\
0.57 & 1.9487 & 26.8952 & 3.5485 \\
0.62 & 2.1674 & 27.5254 & 3.4535 \\
0.67 & 2.3921 & 28.1328 & 3.3657 \\
0.72 & 2.6226 & 28.7200 & 3.2840 \\
0.77 & 2.8588 & 29.2892 & 3.2076 \\
0.82 & 3.1006 & 29.8422 & 3.1359 \\
0.87 & 3.3478 & 30.3806 & 3.0684 \\
0.92 & 3.6003 & 30.9057 & 3.0046 \\
0.97 & 3.8580 & 31.4186 & 2.9442 \\
\hline
\end{tabular}

$$
\left.+\frac{(\mathbb{G}(0.99)-\mathbb{G}(0.02))^{1.53}}{\Gamma(2.53)}\right)=30.099324
$$

We consider $B=100$. Then, substituting (69), (74), and (75) into inequality (24), we obtain

$$
\begin{aligned}
\Lambda_{3}+\mathcal{O}_{3} \varrho_{0}^{*} f(B) & =30.099324+3.866648 \times 0.099 \times f(100) \\
& =32.651312<100=B .
\end{aligned}
$$

Hence (C3) holds for $q=q_{3}=0.89$. Tables 3,4 , and 5 show the numerical values of $\mathcal{O}_{j}, \Lambda_{j}$, and $\frac{B}{\Lambda_{j}+\mathcal{O}_{j} \varrho_{0}^{*} f(B)}$ for $\mathfrak{t} \in[0.02,0.99]$ and $q_{j} \in\{0.28,0.53,0.89\}, j=1,2,3$.

These results are also plotted in Fig. 4. In all three cases for the order $q_{i}$, we see that all requirements of Theorem 3.3 are fulfilled. Therefore this guarantees that for all three different cases by terms of the order $q$, the fractional $\mathbb{G}$-snap system (67) admits at least one solution on the interval $[0.02,0.99]$.

Example 6.3 Based on system (46), we consider the nonlinear fractional inclusion system

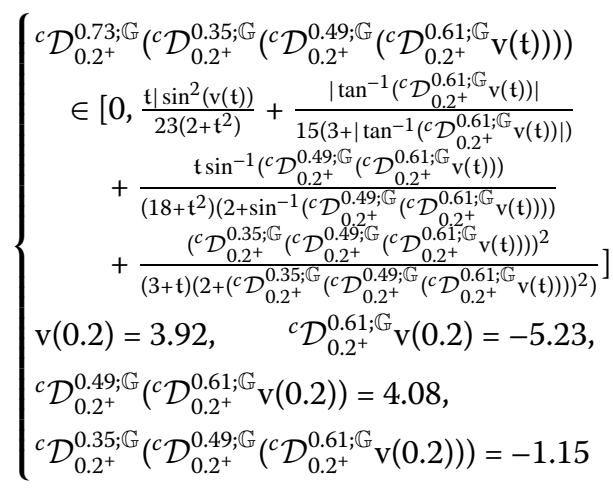


Table 4 Numerical results of $\mathcal{O}_{i}$ and $\Lambda_{i}, i=1,2,3$, for $\mathfrak{t} \in[0.02,0.99]$ in Example 6.2 when $q_{1}=0.28$, $q_{2}=0.53$, and $q_{3}=0.89$

\begin{tabular}{llll}
\hline & \multicolumn{2}{l}{$q_{1}=0.28$} & \\
\cline { 2 - 4 } $\mathfrak{t}$ & $\mathcal{O}_{1}$ & $\Lambda_{1}$ & $\frac{B}{\Lambda_{1}+\mathcal{O}_{1} \varrho_{0}^{*} f(B)}>1$ \\
\hline 0.02 & 0.000000 & 11.480000 & 8.710801 \\
0.07 & 0.138112 & 15.656301 & 6.350232 \\
0.12 & 0.276248 & 17.244645 & 5.738232 \\
0.17 & 0.422128 & 18.506034 & 5.323499 \\
0.22 & 0.576067 & 19.603570 & 5.004060 \\
0.27 & 0.737980 & 20.598497 & 4.742581 \\
0.32 & 0.907712 & 21.521621 & 4.520650 \\
0.37 & 1.085108 & 22.390979 & 4.327665 \\
0.42 & 1.270020 & 23.218193 & 4.156897 \\
0.47 & 1.462316 & 24.011259 & 4.003782 \\
0.52 & 1.661876 & 24.775955 & 3.865064 \\
0.57 & 1.868592 & 25.516610 & 3.738334 \\
0.62 & 2.082367 & 26.236568 & 3.621754 \\
0.67 & 2.303113 & 26.938475 & 3.513885 \\
0.72 & 2.530749 & 27.624462 & 3.413580 \\
0.77 & 2.765202 & 28.296281 & 3.319908 \\
0.82 & 3.006403 & 28.955388 & 3.232102 \\
0.87 & 3.254289 & 29.603011 & 3.149523 \\
0.92 & 3.508804 & 30.240193 & 3.071630 \\
0.97 & 3.769892 & 30.867835 & 2.997965 \\
\hline
\end{tabular}

Table 5 Numerical results of $\mathcal{O}_{i}$ and $\Lambda_{i}, i=1,2,3$, for $\mathfrak{t} \in[0.02,0.99]$ in Example 6.2 when $q_{1}=0.28$, $q_{2}=0.53$, and $q_{3}=0.89$

\begin{tabular}{|c|c|c|c|}
\hline \multirow[b]{2}{*}{$\mathfrak{t}$} & \multicolumn{3}{|l|}{$q_{1}=0.28$} \\
\hline & $\overline{\mathcal{O}_{1}}$ & $\Lambda_{1}$ & $\frac{B}{\Lambda_{1}+\mathcal{O}_{1} \varrho_{0}^{*} f(B)}>1$ \\
\hline 0.02 & 0.000000 & 11.480000 & 8.710801 \\
\hline 0.07 & 0.136126 & 14.719326 & 6.752573 \\
\hline 0.12 & 0.269336 & 15.959688 & 6.196766 \\
\hline 0.17 & 0.408139 & 16.987999 & 5.794625 \\
\hline 0.22 & 0.553358 & 17.917221 & 5.469730 \\
\hline 0.27 & 0.705303 & 18.788358 & 5.193764 \\
\hline 0.32 & 0.864149 & 19.621462 & 4.952505 \\
\hline 0.37 & 1.030023 & 20.427978 & 4.737587 \\
\hline 0.42 & 1.203031 & 21.215104 & 4.543574 \\
\hline 0.47 & 1.383268 & 21.987675 & 4.366692 \\
\hline 0.52 & 1.570824 & 22.749106 & 4.204180 \\
\hline 0.57 & 1.765784 & 23.501897 & 4.053948 \\
\hline 0.62 & 1.968230 & 24.247935 & 3.914359 \\
\hline 0.67 & 2.178243 & 24.988679 & 3.784106 \\
\hline 0.72 & 2.395902 & 25.725280 & 3.662122 \\
\hline 0.77 & 2.621284 & 26.458658 & 3.547520 \\
\hline 0.82 & 2.854465 & 27.189562 & 3.439557 \\
\hline 0.87 & 3.095519 & 27.918608 & 3.337600 \\
\hline 0.92 & 3.344520 & 28.646309 & 3.241103 \\
\hline 0.97 & 3.601540 & 29.373093 & 3.149595 \\
\hline 1.02 & 3.866649 & 30.099324 & 3.062664 \\
\hline
\end{tabular}

for $\mathfrak{t} \in[0.2,0.85]$. It is clear that $a=0.2, b=0.85, q=0.61 \in(0,1], \mathrm{v}(0.2)=\mathrm{v}_{0}=3.92, p=$ $0.49 \in(0,1], \mathrm{u}(0.2)=\mathrm{v}_{1}=-5.23, r=0.35 \in(0,1], \mathrm{w}(0.2)=\mathrm{v}_{2}=4.08, k=0.73 \in(0,1], \mathrm{x}(0)=$ $\mathrm{v}_{3}=-1.15$, and

$$
\left.\widehat{\mathfrak{H}}_{\mathrm{v}}(\mathfrak{t})=\mathfrak{H}\left(\mathfrak{t}, \mathrm{v}_{1}, \mathrm{v}_{2}, \mathrm{v}_{3}, \mathrm{v}_{4}\right)\right)
$$




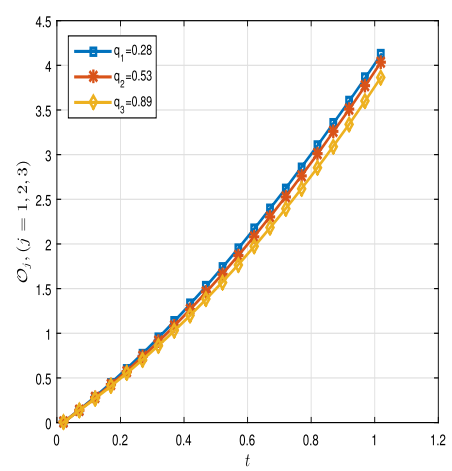

(a) $\mathcal{O}_{j}(j=1,2,3)$

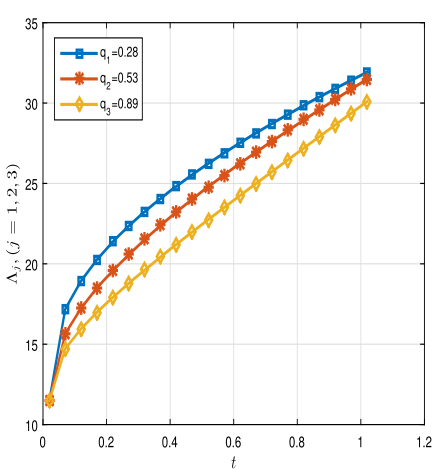

(b) $\Lambda_{j}(j=1,2,3)$

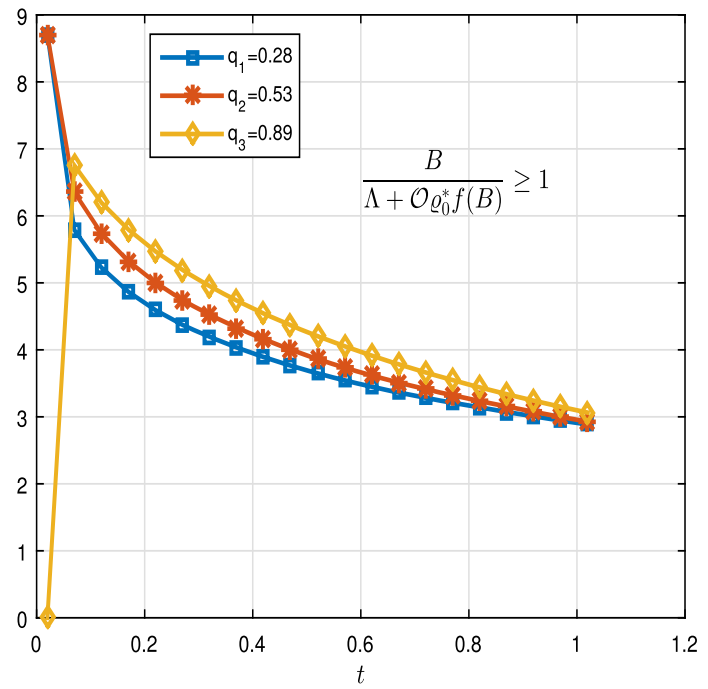

(c) $\frac{K}{\Lambda_{j}+\Delta_{j} \phi_{0}^{*} \varphi(K)}(j=1,2,3)$

Figure 4 Graphical representation of $\Delta_{j}, \Lambda_{j}$, and $\frac{K}{\Lambda_{j}+\Delta_{j} \phi_{0}^{*} \varphi(K)}$ for $\mathfrak{t} \in[0.05,0.95], j=1,2,3$, in Example 6.2 where $q_{1}=0.28, q_{2}=0.53$, and $q_{3}=0.89$

$$
\begin{aligned}
= & {\left[0, \frac{\mathfrak{t} \mid \sin ^{2}\left(\mathrm{v}_{1}(\mathfrak{t})\right)}{23\left(2+\mathfrak{t}^{2}\right)}+\frac{\left|\tan ^{-1}\left(\left(\mathrm{v}_{2}(\mathfrak{t})\right)\right)\right|}{15\left(3+\left|\tan ^{-1}\left(\left(\mathrm{v}_{2}(\mathfrak{t})\right)\right)\right|\right)}\right.} \\
& \left.+\frac{\mathfrak{t} \sin ^{-1}\left(\left(\mathrm{v}_{3}(\mathfrak{t})\right)\right)}{\left(18+\mathfrak{t}^{2}\right)\left(2+\sin ^{-1}\left(\left(\mathrm{v}_{3}(\mathfrak{t})\right)\right)\right)}+\frac{\left(\left(\mathrm{v}_{4}(\mathfrak{t})\right)\right)^{2}}{(3+\mathfrak{t})\left(2+\left(\left(\mathrm{v}_{4}(\mathfrak{t})\right)\right)^{2}\right)}\right]
\end{aligned}
$$

For, $\mathrm{v}_{j}, \mathbf{v}_{j} \in \mathcal{C}(j=1,2,3,4)$, we have

$$
\begin{aligned}
\mathcal{H}_{d}\left(\mathfrak{H}\left(\mathrm{t}, \mathrm{v}_{1}, \mathrm{v}_{2}, \mathrm{v}_{3}, \mathrm{v}_{4}\right), \mathfrak{H}\left(\mathrm{t}, \mathrm{v}_{1}^{\prime}, \mathrm{v}_{2}^{\prime}, \mathrm{v}_{3}^{\prime}, \mathrm{v}_{4}^{\prime}\right)\right) \\
\leq \frac{\mathfrak{t}}{4}\left(\frac{1}{2}\left|\sin \left(\mathrm{v}_{1}(\mathfrak{t})\right)-\sin \left(\mathrm{v}_{1}^{\prime} \mathrm{t}\right)\right|+\frac{1}{2}\left|\tan ^{-1}\left(\mathrm{v}_{2}(\mathfrak{t})\right)-\tan ^{-1}\left(\mathrm{v}_{2}(\mathfrak{t})\right)\right|\right. \\
\left.\quad+\frac{1}{2}\left|-\sin ^{-1}\left(\mathrm{v}_{3}(\mathfrak{t})\right) \sin ^{-1}\left(\mathrm{v}_{3}^{\prime}(\mathfrak{t})\right)\right|+\frac{1}{2}\left|\mathrm{v}_{4}(\mathfrak{t})-\mathrm{v}_{4}(\mathfrak{t})\right|\right)
\end{aligned}
$$




$$
\leq \phi(\mathfrak{t}) \mathcal{O}^{*} \psi\left(\sum_{j=1}^{4}\left|\mathrm{v}_{j}-\mathfrak{v}_{j}\right|\right) .
$$

Now we consider four cases for $\mathbb{G}$ :

$$
\mathbb{G}_{1}(\mathfrak{t})=2^{\mathfrak{t}}, \quad \mathbb{G}_{2}(\mathfrak{t})=\mathfrak{t}, \quad \mathbb{G}_{3}(\mathfrak{t})=\ln \mathfrak{t}, \quad \mathbb{G}_{4}(\mathfrak{t})=\sqrt{\mathfrak{t}} .
$$

Note that $\mathbb{G}_{2}, \mathbb{G}_{3}$, and $\mathbb{G}_{4}$ give the Caputo, Caputo-Hadamard, and Katugampola (for $\rho=0.5)$ derivatives in this example. By equation (12) we have

$$
\begin{aligned}
\mathcal{O}^{*}=\mathcal{O}^{-1}:= & {\left[\frac{(\mathbb{G}(b)-\mathbb{G}(a))^{q+p+r+k}}{\Gamma(q+p+r+k+1)}+\frac{(\mathbb{G}(b)-\mathbb{G}(a))^{p+r+k}}{\Gamma(p+r+k+1)}\right.} \\
& \left.+\frac{(\mathbb{G}(b)-\mathbb{G}(a))^{r+k}}{\Gamma(r+k+1)}+\frac{(\mathbb{G}(b)-\mathbb{G}(a))^{k}}{\Gamma(k+1)}\right]^{-1} \\
= & {\left[\frac{(\mathbb{G}(0.85)-\mathbb{G}(0.2))^{2.18}}{\Gamma(3.18)}+\frac{(\mathbb{G}(0.85)-\mathbb{G}(0.2))^{1.57}}{\Gamma(2.57)}\right.} \\
& \left.+\frac{(\mathbb{G}(0.85)-\mathbb{G}(0.2))^{1.08}}{\Gamma(2.08)}+\frac{(\mathbb{G}(0.85)-\mathbb{G}(0.2))^{0.73}}{\Gamma(1.73)}\right]^{-1} .
\end{aligned}
$$

Therefore

$$
\mathcal{O}^{*}=0.458030,0.461510,0.150228,0.685475
$$

for $\mathbb{G}_{j}(\mathfrak{t})(j=1,2,3,4)$, respectively. Choose the nonnegative function $\phi \in C([a, b],[0, \infty))$ defined by $\phi(\mathfrak{t})=\frac{\mathfrak{t}}{4}$ for $\mathfrak{t} \in[a, b]$. Then $\|\phi\|=0.2125$. Also, we consider the nonnegative nondecreasing u.s.c map $\psi: \mathbb{R}_{\geq 0} \rightarrow \mathbb{R}_{\geq 0}$ defined by $\psi(\mathfrak{t})=\frac{\mathfrak{t}}{2}$ for almost all $\mathfrak{t}>0$. Note that $\lim _{\mathfrak{t} \rightarrow \infty} \inf (\mathfrak{t}-\psi(\mathfrak{t}))>0$ with $\psi(\mathfrak{t})<\mathfrak{t}(\forall \mathfrak{t}>0)$. Finally, consider $\mathfrak{U}: \mathcal{C} \rightarrow \mathcal{P}(\mathcal{C})$ by

$$
\mathfrak{U}(\mathrm{v}):=\left\{\mathfrak{p} \in \mathcal{C}: \text { there exists } \wp \in \mathfrak{S}_{\mathfrak{H}, \mathrm{v}} \text { s.t.p }(\mathfrak{t})=\Upsilon(\mathfrak{t}) \forall \mathfrak{t} \in[a, b]\right\}
$$

where we have

$$
\begin{aligned}
\Upsilon(\mathfrak{t})= & \mathrm{v}_{0}+\frac{\mathrm{v}_{1}(\mathbb{G}(\mathfrak{t})-\mathbb{G}(a))^{q}}{\Gamma(q+1)}+\frac{\mathrm{v}_{2}(\mathbb{G}(\mathfrak{t})-\mathbb{G}(a))^{q+p}}{\Gamma(q+p+1)} \\
& +\frac{\mathrm{v}_{3}(\mathbb{G}(\mathfrak{t})-\mathbb{G}(a))^{q+p+r}}{\Gamma(q+p+r+1)} \\
& +\int_{a}^{\mathfrak{t}} \mathbb{G}^{\prime}(\xi) \frac{(\mathbb{G}(\mathfrak{t})-\mathbb{G}(\xi))^{q+p+r+k-1}}{\Gamma(q+p+r+k)} \wp(\xi) \mathrm{d} \xi \\
= & 3.92+\frac{(-5.23)(\mathbb{G}(\mathfrak{t})-\mathbb{G}(0.2))^{0.61}}{\Gamma(1.61)}+\frac{4.08(\mathbb{G}(\mathfrak{t})-\mathbb{G}(0.2))^{1.1}}{\Gamma(2.1)} \\
& +\frac{(-1.15)(\mathbb{G}(\mathfrak{t})-\mathbb{G}(0.2))^{1.45}}{\Gamma(2.45)} \\
& +\int_{0.2}^{\mathfrak{t}} \mathbb{G}^{\prime}(\xi) \frac{(\mathbb{G}(\mathfrak{t})-\mathbb{G}(\xi))^{1.18}}{\Gamma(2.18)} \wp(\xi) \mathrm{d} \xi .
\end{aligned}
$$

Considering $\wp=\frac{t}{10}$, we can see the results of $\Upsilon(\mathfrak{t})$ in Table 6 . These results are plotted in Fig. 5. Since the operator $\mathfrak{U}$ has the (AEP)-property, by Theorem 5.2 system (76) has at 
Table 6 Numerical results of $\mathcal{O}_{j}^{*}$ and $\Upsilon_{j}, j=1,2,3,4$, for $\mathfrak{t} \in[0.2,0.85]$ in Example 6.3 when $\mathbb{G}_{1}(\mathfrak{t})=2^{\mathfrak{t}}$, $\mathbb{G}_{2}(\mathfrak{t})=\mathfrak{t}, \mathbb{G}_{3}(\mathfrak{t})=\ln \mathfrak{t}, \mathbb{G}_{4}(\mathfrak{t})=\sqrt{\mathfrak{t}}$

\begin{tabular}{|c|c|c|c|c|c|c|c|c|}
\hline & \multicolumn{2}{|l|}{$\mathbb{G}_{1}(\mathfrak{t})$} & \multicolumn{2}{|l|}{$\mathbb{G}_{2}(\mathfrak{t})$} & \multicolumn{2}{|l|}{$\mathbb{G}_{3}(\mathfrak{t})$} & \multicolumn{2}{|l|}{$\mathbb{G}_{4}(\mathfrak{t})$} \\
\hline & $\overline{\mathcal{O}^{*}(\mathfrak{t})}$ & $\Upsilon(\mathfrak{t})$ & $\overline{\mathcal{O}^{*}(\mathfrak{t})}$ & $\Upsilon(\mathfrak{t})$ & $\overline{\mathcal{O}^{*}(\mathfrak{t})}$ & $\Upsilon(\mathfrak{t})$ & $\overline{\mathcal{O}^{*}(\mathfrak{t})}$ & $\Upsilon(\mathfrak{t})$ \\
\hline 0.30 & 4.4298 & 0.0000 & 3.6823 & 2.7630 & 0.8289 & 2.7643 & 3.6643 & 2.7630 \\
\hline 0.40 & 2.1565 & 0.0002 & 1.8284 & 2.3073 & 0.4244 & 2.3117 & 1.9820 & 2.3072 \\
\hline 0.50 & 1.3460 & 0.0007 & 1.1746 & 1.9969 & 0.2912 & 2.0055 & 1.3782 & 1.9967 \\
\hline 0.60 & 0.9321 & 0.0016 & 0.8424 & 1.7650 & 0.2254 & 1.7782 & 1.0636 & 1.7643 \\
\hline 0.70 & 0.6836 & 0.0031 & 0.6432 & 1.5843 & 0.1863 & 1.6023 & 0.8694 & 1.5828 \\
\hline 0.80 & 0.5200 & 0.0055 & 0.5116 & 1.4406 & 0.1602 & 1.4632 & 0.7371 & 1.4378 \\
\hline 0.81 & 0.5067 & 0.0058 & 0.5009 & 1.4279 & 0.1581 & 1.4509 & 0.7261 & 1.4249 \\
\hline 0.82 & 0.4939 & 0.0061 & 0.4905 & 1.4154 & 0.1560 & 1.4389 & 0.7155 & 1.4123 \\
\hline 0.83 & 0.4815 & 0.0065 & 0.4805 & 1.4033 & 0.1540 & 1.4272 & 0.7052 & 1.4000 \\
\hline 0.84 & 0.4696 & 0.0068 & 0.4709 & 1.3913 & 0.1521 & 1.4157 & 0.6952 & 1.3879 \\
\hline 0.85 & 0.4580 & 0.0072 & 0.4615 & 1.3797 & 0.1502 & 1.4045 & 0.6855 & 1.3761 \\
\hline
\end{tabular}

Figure 5 Graphical representation of $\mathcal{O}_{j}$ and $\Upsilon_{j}$ for $\mathfrak{t} \in[0.2,0.85], j=1,2,3,4$, in Example 6.3 where $\mathbb{G}_{1}(\mathfrak{t})=2^{\mathfrak{t}}$, $\mathbb{G}_{2}(\mathfrak{t})=\mathfrak{t}, \mathbb{G}_{3}(\mathfrak{t})=\ln \mathfrak{t}, \mathbb{G}_{4}(\mathfrak{t})=\sqrt{\mathfrak{t}}$

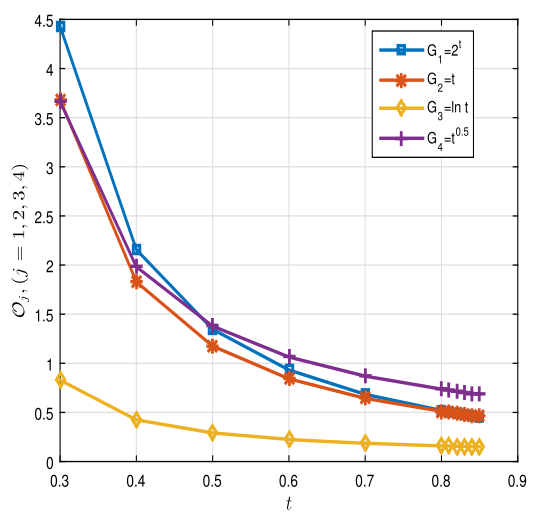

(a) $\mathcal{O}_{j}(j=1,2,3)$

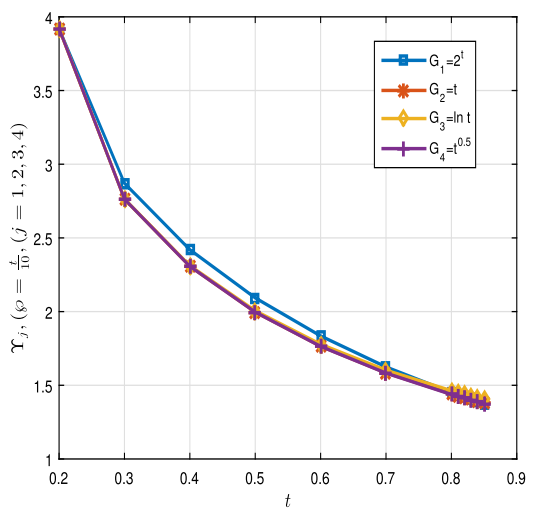

(b) $\Upsilon_{j}$, where $\wp=\frac{1}{10} \mathfrak{t}(j=1,2,3,4)$

least one solution.

\section{Conclusion}

In this paper, we defined a new fractional mathematical model of a BVP consisting of the snap equation in the framework of the generalized sequential $\mathbb{G}$-operators and turned 


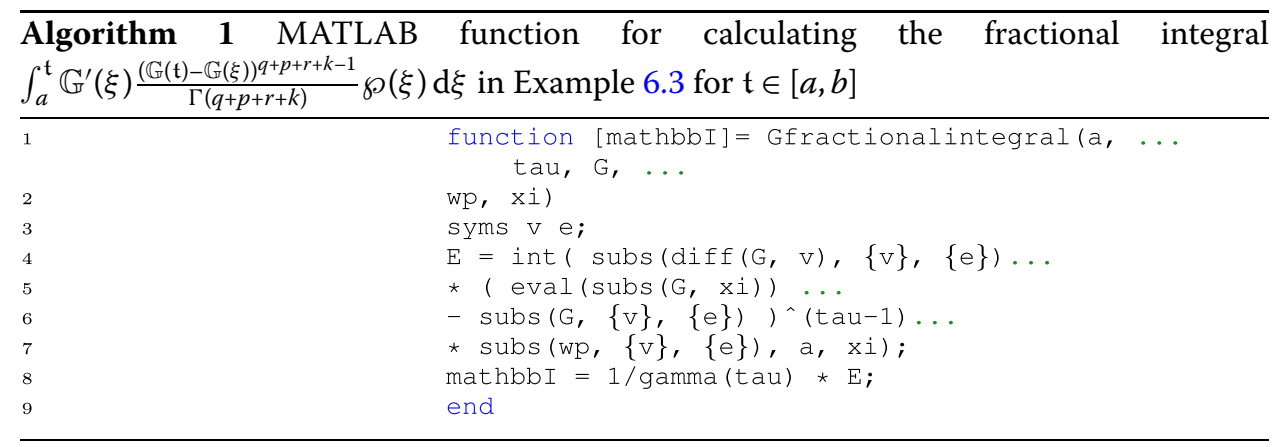

to the investigation of the qualitative behaviors of its solutions including the existence, uniqueness, stability, and inclusion version. To obtain an existence criterion, we used the Leray-Schauder theorem, and to obtain a uniqueness criterion, we utilized the Banach theorem. We studied different kinds of stability criteria based on the standard definitions of these notions. With the help of some special contractions, we established some theorems regarding the inclusion structure of the $\mathbb{G}$-snap problem. In the final step, we designed three examples, and considering different cases of the function $\mathbb{G}$ and order $q$, we obtained numerical results of these two suggested fractional $\mathbb{G}$-snap systems in Caputo, Caputo-Hadamard, and Katugampola versions. Note that in this paper, by assuming $\mathbb{G}(\mathfrak{t})=\mathfrak{t}$ and $q=p=r=k=1$ we derived the standard 4th-order ODE of snap equation. Therefore we will be able to review other properties of this extended fractional $\mathbb{G}$-snap BVP by designing new generalized models based on nonsingular operators in the future works. 


\section{Supporting information}

\section{Algorithm 2 MATLAB lines for calculating values of $\mathcal{O}, L \mathcal{O}, \Lambda$, and $\ell$ in Example 6.1}

for $\mathfrak{t} \in[1.1,2.6]$ and $\mathbb{G}(\mathfrak{t}):=\left\{2^{\mathfrak{t}}, \mathfrak{t}, \ln \mathfrak{t}, \sqrt{\mathfrak{t}}\right\}$

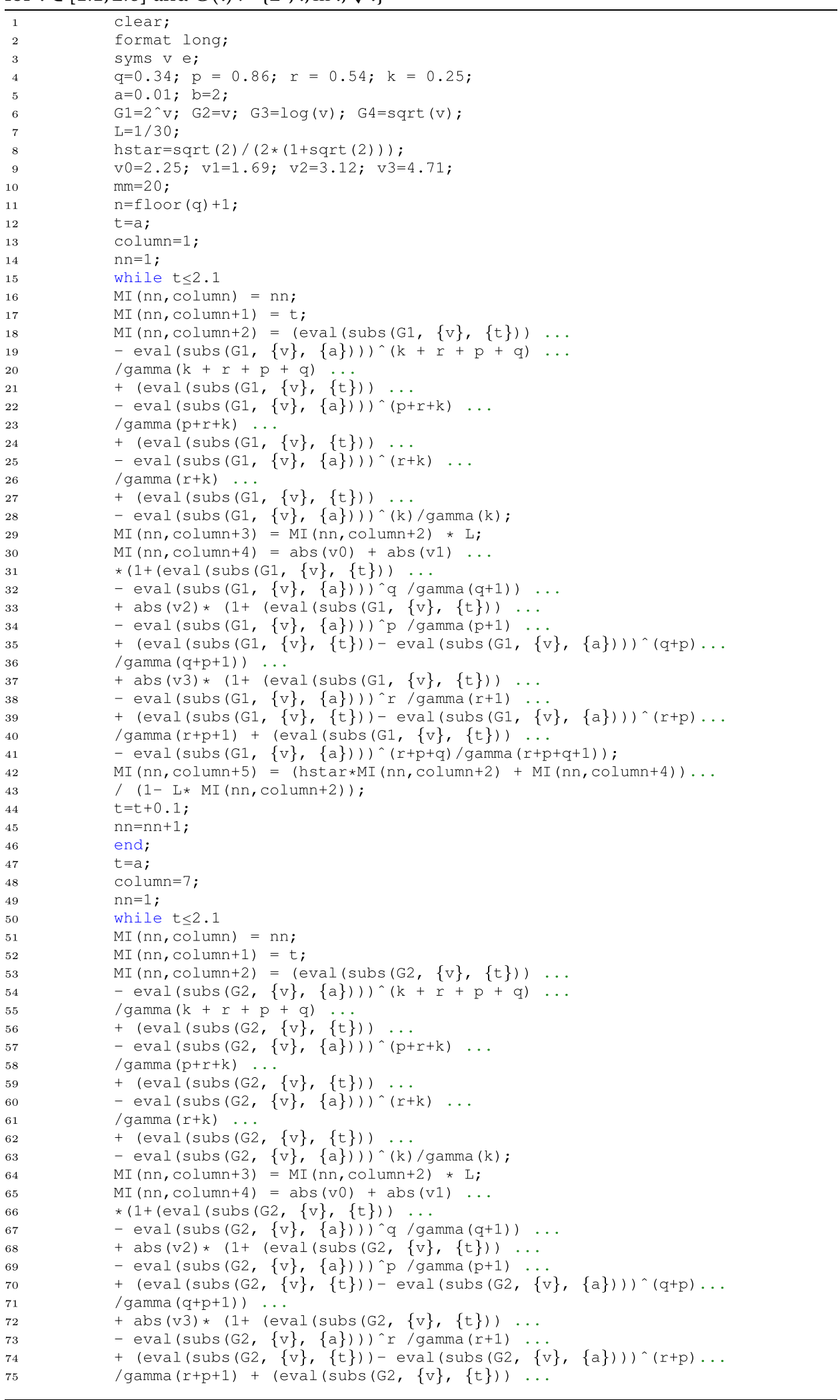




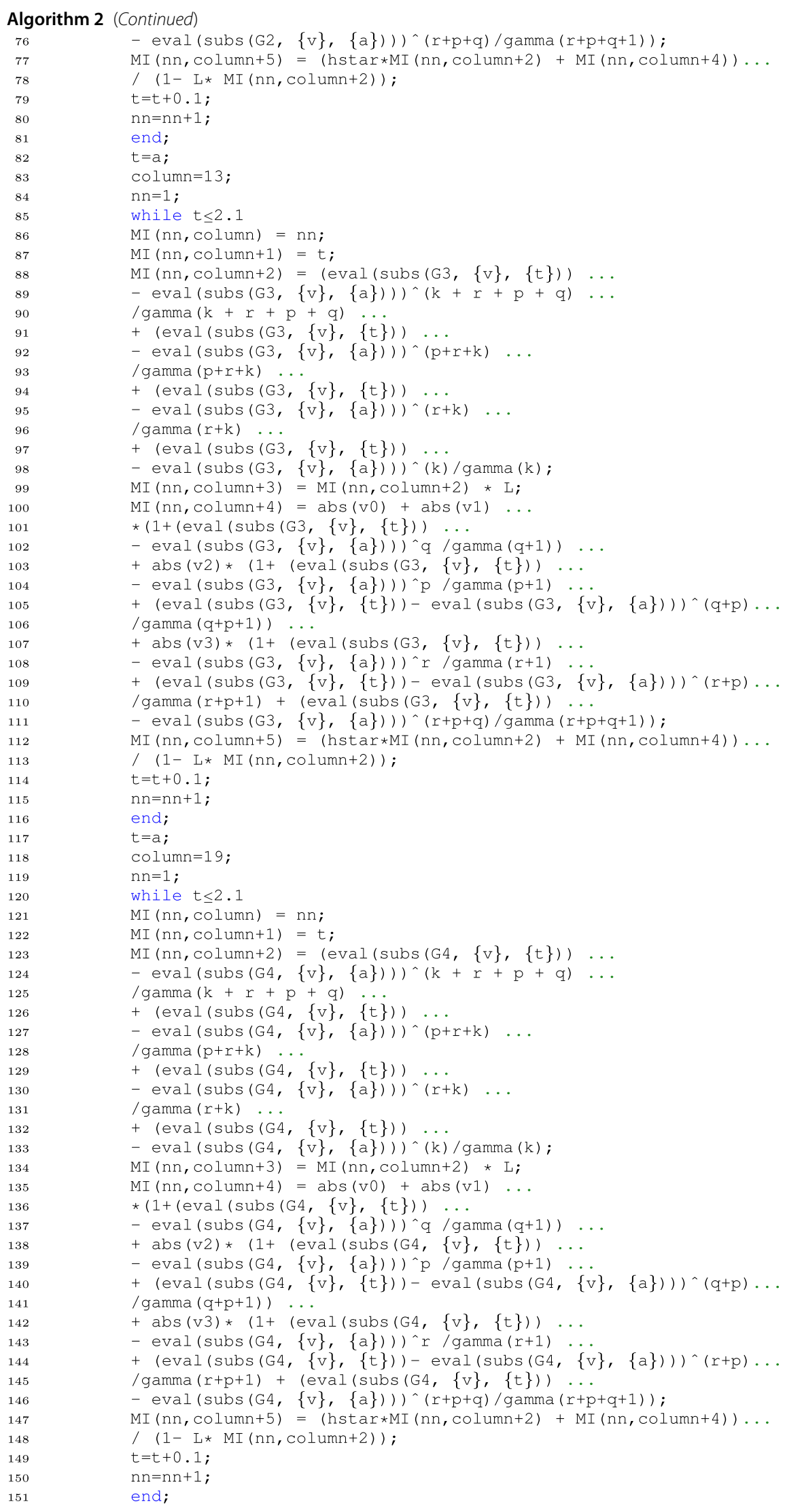




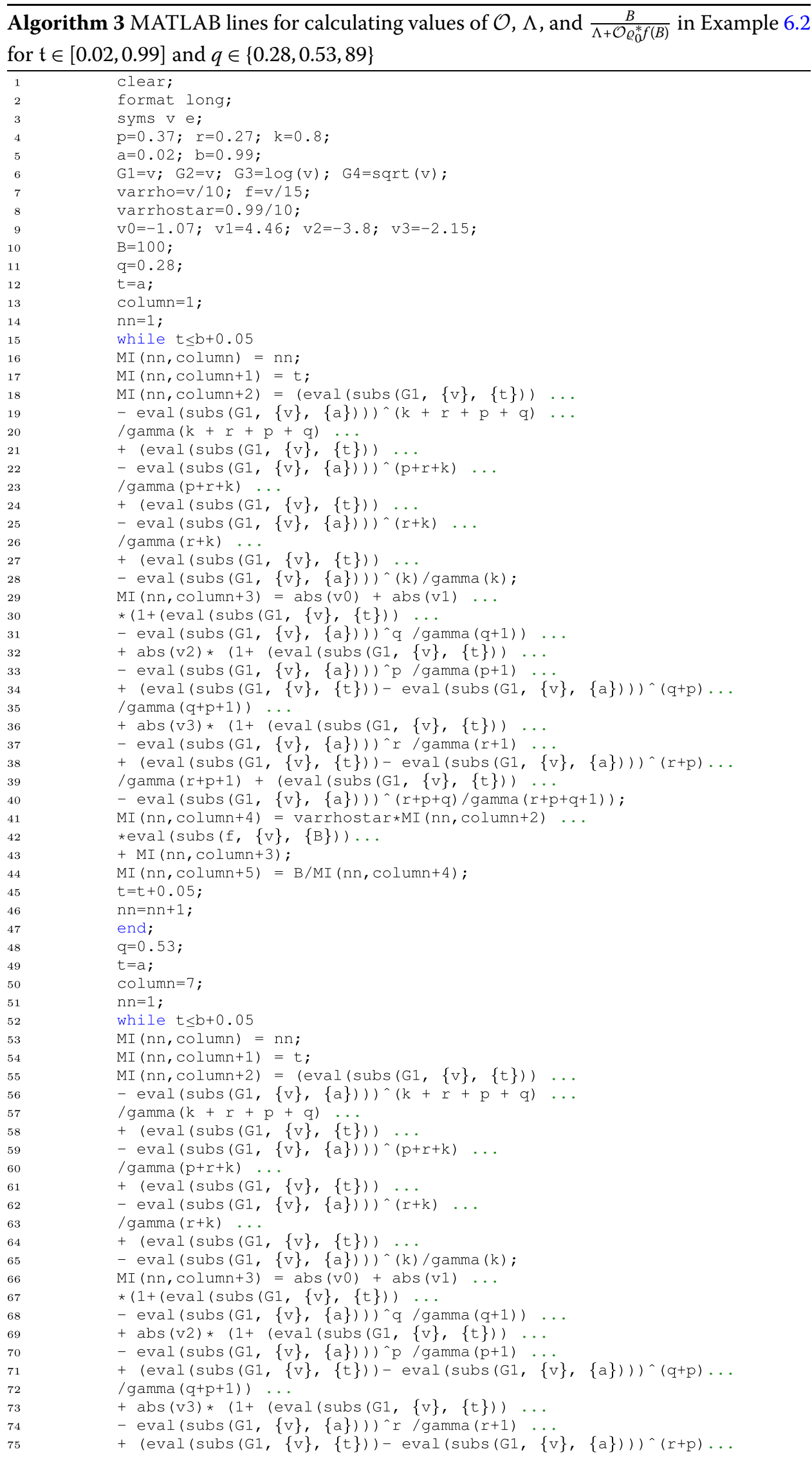




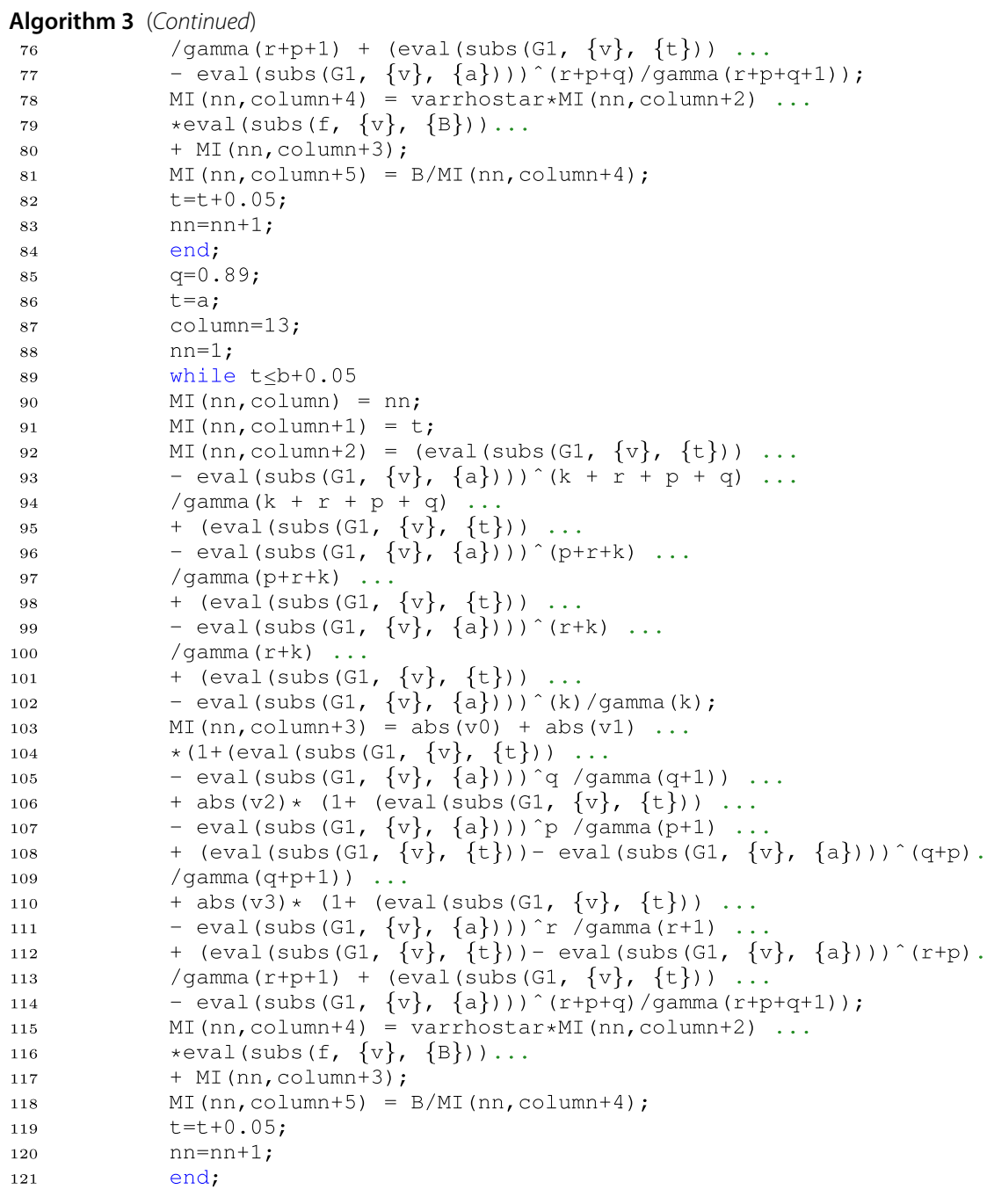


Algorithm 4 MATLAB lines for calculating values of $\mathcal{O}^{*}$ and $\Upsilon$ in Example 6.3 for $\mathfrak{t} \epsilon$ $[0.2,0.85]$ and $\mathbb{G}(\mathfrak{t}):=\left\{2^{\mathfrak{t}}, \mathfrak{t}, \ln \mathfrak{t}, \sqrt{\mathfrak{t}}\right\}$

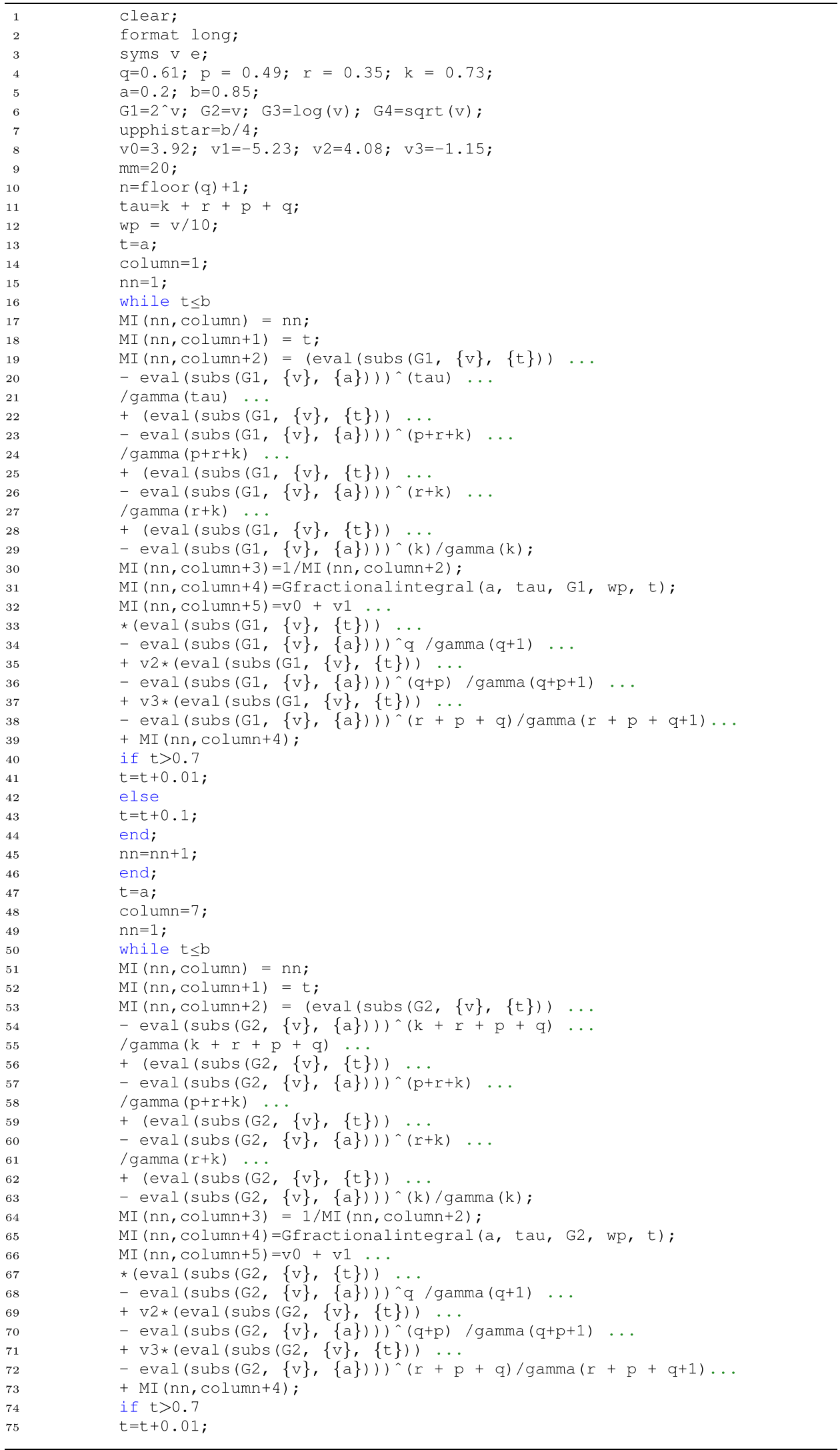




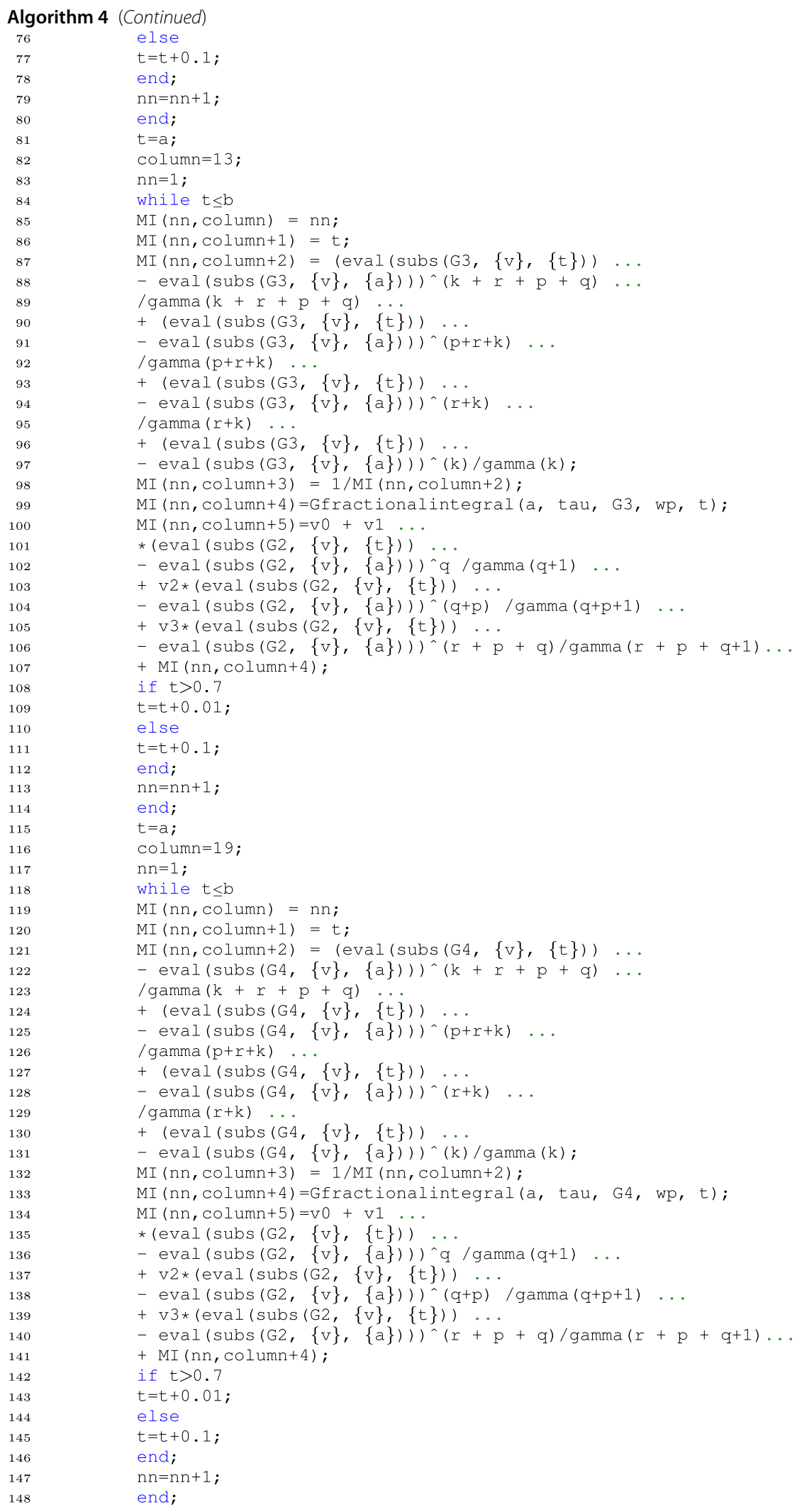




\section{Acknowledgements}

The second and fifth authors were supported by Azarbaijan Shahid Madani University. The third author was supported by Bu-Ali Sina University. The authors express their gratitude to unknown referees for their helpful suggestions, which improved the final version of this paper.

\section{Funding}

Not applicable.

Availability of data and materials

Data sharing not applicable to this paper as no datasets were generated or analyzed during the current study.

\section{Declarations}

\section{Ethics approval and consent to participate}

Not applicable.

\section{Consent for publication}

Not applicable.

\section{Competing interests}

The authors declare that they have no competing interests

\section{Authors' contributions}

The authors declare that the study was realized in collaboration with equal responsibility. All authors read and approved the final manuscript.

\section{Author details}

'Department of Mathematics, Bu-Ali Sina University, 65178, Hamedan, Iran. ${ }^{2}$ Department of Mathematics, Al-Azhar University-Gaza, Gaza Strip, State of Palestine. ${ }^{3}$ Department of Mathematics, Azarbaijan Shahid Madani University, Tabriz, Iran. ${ }^{4}$ Department of Medical Research, China Medical University Hospital, China Medical University, Taichung, Taiwan.

\section{Publisher's Note}

Springer Nature remains neutral with regard to jurisdictional claims in published maps and institutional affiliations.

Received: 31 August 2021 Accepted: 1 November 2021 Published online: 17 November 2021

\section{References}

1. Lazreg, J.E., Abbas, S., Benchohra, M.: Impulsive Caputo-Fabrizio fractional differential equations in b-metric spaces. Open Math. 19(2), 363-372 (2021). https://doi.org/10.1515/math-2021-0040

2. Krim, S., Abbas, S., Benchohra, M.: Terminal value problem for implicit Katugampola fractional differential equations in b-metric spaces. J. Funct. Spaces 2021, Article ID 5535178 (2021). https://doi.org/10.1155/2021/5535178

3. Baitiche, Z., Derbazi, C., Benchohra, M.: $\psi$-Caputo fractional differential equations with multi-point boundary conditions by topological degree theory. Res. Nonlinear Anal. 3(4), 167-178 (2020)

4. Wahash, H.A., Abdo, M., Panchal, S.K.: Existence and stability of a nonlinear fractional differential equation involving a $\psi$-Caputo operator. Adv. Theory Nonlinear Anal. Appl. 4(4), 266-278 (2020). https://doi.org/10.31197/atnaa.664534

5. Pham, V.T., Vaidyanathan, S., Volos, C., Jafari, S., Alsaadi, F.E.: Chaos in a simple snap system with only one nonlinearity, its adaptive control and real circuit design. Arch. Control Sci. 29(1), 73-96 (2019). https://doi.org/10.1186/1687-1847-2012-140

6. Baitiche, Z., Derbazi, C., Matar, M.M.: Ulam stability for nonlinear Langevin fractional differential equations involving two fractional orders in the $\psi$-Caputo sense. Appl. Anal. 2021, 1-16 (2021). https://doi.org/10.1080/00036811.2021.1873300

7. Etemad, S., Rezapour, S., Samei, M.E.: On a fractional Caputo-Hadamard inclusion problem with sum boundary value conditions by using approximate endpoint property. Math. Methods Appl. Sci. 43(17), 9719-9734 (2020). https://doi.org/10.1002/mma.6644

8. Boutiara, A., Guerbati, K., Benbachir, M.: Caputo-Hadamard fractional differential equation with three-point boundary conditions in Banach spaces. AIMS Math. 5(1), 259-272 (2020). https://doi.org/10.3934/math.2020017

9. Baleanu, D., Etemad, S., Rezapour, S.: On a fractional hybrid integro-differential equation with mixed hybrid integral boundary value conditions by using three operators. Alex. Eng. J. 59(5), 3019-3027 (2020). https://doi.org/10.1016/j.aej.2020.04.053

10. Mohammadi, H., Kumar, S., Etemad, S., Rezapour, S.: A theoretical study of the Caputo-Fabrizio fractional modeling for hearing loss due to Mumps virus with optimal control. Chaos Solitons Fractals 144, 110668 (2021). https://doi.org/10.1016/j.chaos.2021.110668

11. Thabet, S.T.M., Etemad, S., Rezapour, S.: On a coupled Caputo conformable system of pantograph problems. Turk. J. Math. 45(1), 496-519 (2021). https://doi.org/10.3906/mat-2010-70

12. Mohammadi, H., Baleanu, D., Etemad, S., Rezapour, S.: Criteria for existence of solutions for a Liouville-Caputo boundary value problem via generalized Gronwall's inequality. J. Inequal. Appl. 2021, Article ID 36 (2021). https://doi.org/10.1186/s13660-021-02562-6

13. Boutiara, A., Benbachir, M., Guerbati, K.: Caputo type fractional differential equation with nonlocal Erdelyi-Kober type integral boundary conditions in Banach spaces. Surv. Math. Appl. 15, 399-418 (2020) 
14. Rezapour, S., Imran, A., Hussain, A., Martinez, F., Etemad, S., Kaabar, M.K.A.: Condensing functions and approximate endpoint criterion for the existence analysis of quantum integro-difference FBVPs. Symmetry 13(3), 469 (2021). https://doi.org/10.3390/sym13030469

15. Mahmudov, N., Matar, M.M.: Existence of mild solution for hybrid differential equations with arbitrary order. TWMS J. Pure Appl. Math. 8(2), 160-169 (2017)

16. Rezapour, S., Samei, M.E.: On the existence of solutions for a multi-singular pointwise defined fractional q-integro-differential equation. Bound. Value Probl. 2020, Article ID 38 (2020). https://doi.org/10.1186/s13661-020-01342-3

17. Ullah, A., Shah, K., Abdeljawad, T., Khan, R.A., Mahariq, l.: Study of impulsive fractional differential equation under Robin boundary conditions by topological degree method. Bound. Value Probl. 2020, Article ID 98 (2020). https://doi.org/10.1186/s13661-020-01396-3

18. Adjabi, Y., Samei, M.E., Matar, M.M., Alzabut, J.: Langevin differential equation in frame of ordinary and Hadamard fractional derivatives under three point boundary conditions. AIMS Math. 6(3), 2796-2843 (2021). https://doi.org/10.3934/math.2021171

19. Matar, M.M.: Qualitative properties of solution for hybrid nonlinear fractional differential equations. Afr. Math. 30, 1169-1179 (2019). https://doi.org/10.1007/s13370-019-00710-2

20. Matar, M.M.: Approximate controllability of fractional nonlinear hybrid differential systems via resolvent operators. J. Math. 2019, Article ID 8603878 (2019). https://doi.org/10.1155/2019/8603878

21. Matar, M.M., Alzabut, J.M.I.A., Kaabar, M.K.A., Etemad, S., Rezapour, S.: Investigation of the p-Laplacian nonperiodic nonlinear boundary value problem via generalized Caputo fractional derivatives. Adv. Differ. Equ. 2021, Article ID 69 (2021). https://doi.org/10.1186/s13662-021-03228-9

22. Abdeljawad, T., Agarwal, R.P., Karapinar, E., Kumari, P.S.: Solutions of the nonlinear integral equation and fractional differential equation using the technique of a fixed point with a numerical experiment in extended b-metric space. Symmetry 11(5), 686 (2019). https://doi.org/10.3390/sym11050686

23. Ngoc, T.B., Tri, V.V., Hammouch, Z., Can, N.H.: Stability of a class of problems for time-space fractional pseudo-parabolic equation with datum measured at terminal time. Appl. Numer. Math. 167, 308-329 (2021) https://doi.org/10.1016/j.apnum.2021.05.009

24. Mahmoud, E.E., Trikha, P., Jahanzaib, L.S.: Application of triple compound combination anti-synchronization among parallel fractional snap systems and electronic circuit implementation. Adv. Differ. Equ. 2021, Article ID 211 (2021). https://doi.org/10.1186/s13662-021-03362-4

25. Adiguzel, R.S., Aksoy, U., Karapinar, E., Erhan, I.M.: On the solutions of fractional differential equations via Geraghty type hybrid contractions. Int. J. Appl. Comput. Math. 20(2), 313-333 (2021)

26. Adiguzel, R.S., Aksoy, U., Karapinar, E., Erhan, I.M.: Uniqueness of solution for higher-order nonlinear fractional differential equations with multi-point and integral boundary conditions. Rev. R. Acad. Cienc. Exactas Fís. Nat., Ser. A Mat. 2021, Article ID 155 (2021). https://doi.org/10.1007/s13398-021-01095-3

27. Bachir, F.S., Abbas, S., Benbachir, M., Benchora, M.: Hilfer-Hadamard fractional differential equations; existence and attractivity. Adv. Theory Nonlinear Anal. Appl. 5(1), 49-57 (2021). https://doi.org/10.31197/atnaa.848928

28. Baleanu, D., Jajarmi, A., Mohammadi, H., Rezapour, S.: A new study on the mathematical modelling of human liver with Caputo-Fabrizio fractional derivative. Chaos Solitons Fractals 134, 109705 (2020). https://doi.org/10.1016/j.chaos.2020.109705

29. Karapinar, E., Fulga, A., Rashid, M., Shahid, L., Aydi, H.: Large contractions on quasi-metric spaces with an application to nonlinear fractional differential equations. Mathematics 7(5), 444 (2019). https://doi.org/10.3390/math7050444

30. Hassan, A.M., Karapinar, E., Alsulami, H.H.: Ulam-Hyers stability for MKC mappings via fixed point theory. J. Funct. Spaces 2016, Article ID 9623597 (2016). https://doi.org/10.1155/2016/9623597

31. Alsulami, H.H., Gulyaz, S., Karapinar, E., Erhan, I.: An Ulam stability result on quasi-b-metric-like spaces. Open Math. 14(1), 1087-1103 (2016). https://doi.org/10.1515/math-2016-0097

32. Brzdek, J., Karapinar, E., Petrsel, A.: A fixed point theorem and the Ulam stability in generalized dq-metric spaces. J. Math. Anal. Appl. 467, 501-520 (2018). https://doi.org/10.1016/j.jmaa.2018.07.022

33. Alqahtani, B., Fulga, A., Karapinar, E.: Fixed point results on $\delta$-symmetric quasi-metric space via simulation function with an application to Ulam stability. Mathematics 6(10), 208 (2018). https://doi.org/10.3390/math6100208

34. Karapinar, E., Fulga, A.: An admissible hybrid contraction with an Ulam type stability. Demonstr. Math. 52, 428-436 (2019). https://doi.org/10.1515/dema-2019-0037

35. Bota, M.F., Karapinar, E., Mlesnite, O.: Ulam-Hyers stability results for fixed point problems via $\alpha$ - $\psi$-contractive mapping in b-metric space. Abstr. Appl. Anal. 2013, Article ID 825293 (2013). https://doi.org/10.1155/2013/825293

36. Luc, N.H., Long, L.D., Hang, L.T.D., Baleanu, D., Can, N.H.: Identifying the initial condition for space-fractional Sobolev equation. J. Appl. Anal. Comput. 167, 20 (2021). https://doi.org/10.11948/20200404

37. Aydogan, S.M., Baleanu, D., Mousalou, A., Rezapour, S.: On high order fractional integro-differential equations including the Caputo-Fabrizio derivative. Bound. Value Probl. 2018, Article ID 90 (2018). https://doi.org/10.1186/s13661-018-1008-9

38. Baleanu, D., Rezapour, S., Saberpour, Z:: On fractional integro-differential inclusions via the extended fractional Caputo-Fabrizio derivation. Bound. Value Probl. 2019, Article ID 79 (2019). https://doi.org/10.1186/s13661-019-1194-0

39. Tuan, N.H., Mohammadi, H., Rezapour, S.: A mathematical model for Covid-19 transmission by using the Caputo fractional derivative. Chaos Solitons Fractals 134, 7 (2020)

40. Baleanu, D., Etemad, S., Rezapour, S.: A hybrid Caputo fractional modeling for thermostat with hybrid boundary value conditions. Bound. Value Probl. 2020, Article ID 64 (2020). https://doi.org/10.1186/s13661-020-01361-0

41. Baleanu, D., Mousalou, A., Rezapour, S.: On the existence of solutions for some infinite coefficient-symmetric Caputo-Fabrizio fractional integro-differential equations. Bound. Value Probl. 2017, Article ID 145 (2017). https://doi.org/10.1186/s13661-017-0867-9

42. Samko, S.G., Kilbas, A.A., Marichev, O.I.: Fractional Integrals and Derivatives: Theory and Applications. Gordon \& Breach, Switzerland (1993)

43. Kilbas, A.A., Srivastava, H.M., Trujillo, J.J.: Theory and Applications of Fractional Differential Equations. North-Holland Mathematics Studies. Elsevier, Amsterdam (2006) 
44. Almeida, R:: A Caputo fractional derivative of a function with respect to another function. Commun. Nonlinear Sci. Numer. Simul. 44, 460-481 (2017). https://doi.org/10.1016/j.cnsns.2016.09.006

45. Almeida, R., Malinowska, A.B., Teresa, M., Monteiro, T.: Fractional differential equations with a Caputo derivative with respect to a kernel function and their applications. Math. Methods Appl. Sci. 41(1), 336-352 (2018). https://doi.org/10.1002/mma.4617

46. Granas, A., Dugundji, J.: Fixed Point Theory. Springer, New York (2003)

47. Amini-Harandi, A.: Endpoints of set-valued contractions in metric spaces. Nonlinear Anal., Theory Methods Appl. 72(1), 132-134 (2010). https://doi.org/10.1016/j.na.2009.06.074

48. Samet, B., Vetro, C., Vetro, P.: Fixed point theorems for $\alpha-\psi$-contractive type mappings. Nonlinear Anal., Theory Methods Appl. 75(4), 2154-2165 (2018). https://doi.org/10.1016/j.na.2011.10.014

49. Mohammadi, B., Rezapour, S., Shahzad, N.: Some results on fixed points of $\alpha$ - $\psi$-Ciric generalized multifunctions. Fixed Point Theory Appl. 2013, Article ID 24 (2013). https://doi.org/10.1 186/1687-1812-2013-24

50. Smart, D.R.: Fixed Point Theorems. Cambridge University Press, Cambridge (1980)

Submit your manuscript to a SpringerOpen ${ }^{\circ}$ journal and benefit from:

- Convenient online submission

- Rigorous peer review

- Open access: articles freely available online

- High visibility within the field

- Retaining the copyright to your article

Submit your next manuscript at $\boldsymbol{\nabla}$ springeropen.com 